\title{
The Botanical, Chemical and Ethnobotanical Diversity of Southern African Lamiaceae
}

\author{
Ryan D. Rattray and Ben-Erik Van Wyk *
}

Citation: Rattray, R.D.; Van Wyk, B.-E. The Botanical, Chemical and Ethnobotanical Diversity of Southern African Lamiaceae. Molecules 2021, 26, 3712. https://doi.org/10.3390/ molecules 26123712

Academic Editors: İhsan Çalış, Horváth Györgyi and Agnieszka Ludwiczuk

Received: 20 May 2021

Accepted: 11 June 2021

Published: 18 June 2021

Publisher's Note: MDPI stays neutral with regard to jurisdictional claims in published maps and institutional affiliations.

Copyright: (c) 2021 by the authors. Licensee MDPI, Basel, Switzerland. This article is an open access article distributed under the terms and conditions of the Creative Commons Attribution (CC BY) license (https:/ / creativecommons.org/licenses/by/ $4.0 /)$.
Department of Botany and Plant Biotechnology, University of Johannesburg, Auckland Park, P.O. Box 524, Johannesburg 2006, South Africa; ryanr@uj.ac.za

* Correspondence: bevanwyk@uj.ac.za; Tel.: +27-11-559-2412

\begin{abstract}
The Lamiaceae is undoubtedly an important plant family, having a rich history of use that spans the globe with many species being used in folk medicine and modern industries alike. Their ability to produce aromatic volatile oils has made them valuable sources of materials in the cosmetic, culinary, and pharmaceutical industries. A thorough account of the taxonomic diversity, chemistry and ethnobotany is lacking for southern African Lamiaceae, which feature some of the region's most notable medicinal and edible plant species. We provide a comprehensive insight into the Lamiaceae flora of southern Africa, comprising 297 species in 42 genera, 105 of which are endemic to the subcontinent. We further explore the medicinal and traditional uses, where all genera with documented uses are covered for the region. A broad review of the chemistry of southern African Lamiaceae is presented, noting that only 101 species (34\%) have been investigated chemically (either their volatile oils or phytochemical characterization of secondary metabolites), thus presenting many and varied opportunities for further studies. The main aim of our study was therefore to present an up-to-date account of the botany, chemistry and traditional uses of the family in southern Africa, and to identify obvious knowledge gaps.
\end{abstract}

Keywords: Lamiaceae; diversity; southern Africa; volatile oils; essential oils; phytochemistry; traditional medicines; useful plants; medicinal plants; food plants

\section{Introduction}

The Lamiaceae Martinov (formerly Labiate) are a commercially important, cosmopolitan family of flowering plants comprising ca. 250 genera and 7825 species [1]. The largest and best-known genera include Salvia L. (900 spp.), Scutellaria L. (360 spp.), Stachys L. (300 spp.), Plectranthus L'Hér. (300 spp.), Hyptis Jacq. (280 spp.), Teucrium L. (250 spp.), Vitex L. (250 spp.), Thymus L. (220 spp.) and Nepeta L. (200 spp.). Many species are highly aromatic and possess complex mixtures of bioactive secondary metabolites which contribute to their global importance as sources of medicinal and culinary herbs. Herbal teas that are rich in aromatic and phenolic compounds, such as chamomile (Matricaria chamomilla L.), rooibos tea (Aspalathus linearis (Burm.f.) Dahlg.), maté (Ilex paraguariensis A.St.-Hil.) and green tea (Camellia sinensis (L.) Kuntze) have become very popular [2,3] and it seems worthwhile to systematically explore the commercial potential of poorly known herbal teas.

A recent global review of ca. 900 commercially important medicinal plant species [2] included 44 members of the Lamiaceae (ca. 5\% of the total). Similarly, a review of major food plants [3] listed 817 species, of which 43 (ca. 5\%) belongs to the Lamiaceae. The contribution of Lamiaceae species to the culinary herbs and spices of the world is even more prominent with 114 species out of 701 listed (ca. 16\%) [4]. The Lamiaceae also features prominently in a family-level analysis of medicinal plants used in Traditional African medicine [5]. Due to their popular use, members of this family play a pivotal role in many industries, including cosmetics, flavoring, fragrance, perfumery, pesticides and pharmaceutical development. On account of their economic importance and wide-spread 
use, many species have been introduced and cultivated in various countries across the world [6].

Southern Africa is not only known for its high levels of plant diversity (20,401 species) and plant endemism (67\%) [7] but also for its cultural diversity, resulting in a rich ethnobotanical heritage. Many important aromatic, medicinal and ornamental plants have been used for centuries in traditional medicines by indigenous people across the globe [8]. A bibliography of plants used in traditional medicine in southern Africa [Arnold et al. (2002)] included 215 families, equating to 3689 taxa (comprising species, subspecies and varieties) and represents ca. $15 \%$ of the regional flora [9]. Some southern African representatives of the Lamiaceae are well-documented as traditional medicines, including Leonotis leonurus (L.) R. Br. and Salvia africana L., as well as food and beverage plants (Coleus esculentus (N.E. Br.) G. Taylor, C. rotundifolius (Poir.) A. Chev. and Perrot and Mentha aquatica L.) [10-14]. In a review of 150 important medicinal plants of South Africa (Van Wyk et al. (2009) [15]), 15 species of Lamiaceae that are commonly used in traditional medicine were included (Figure 1).

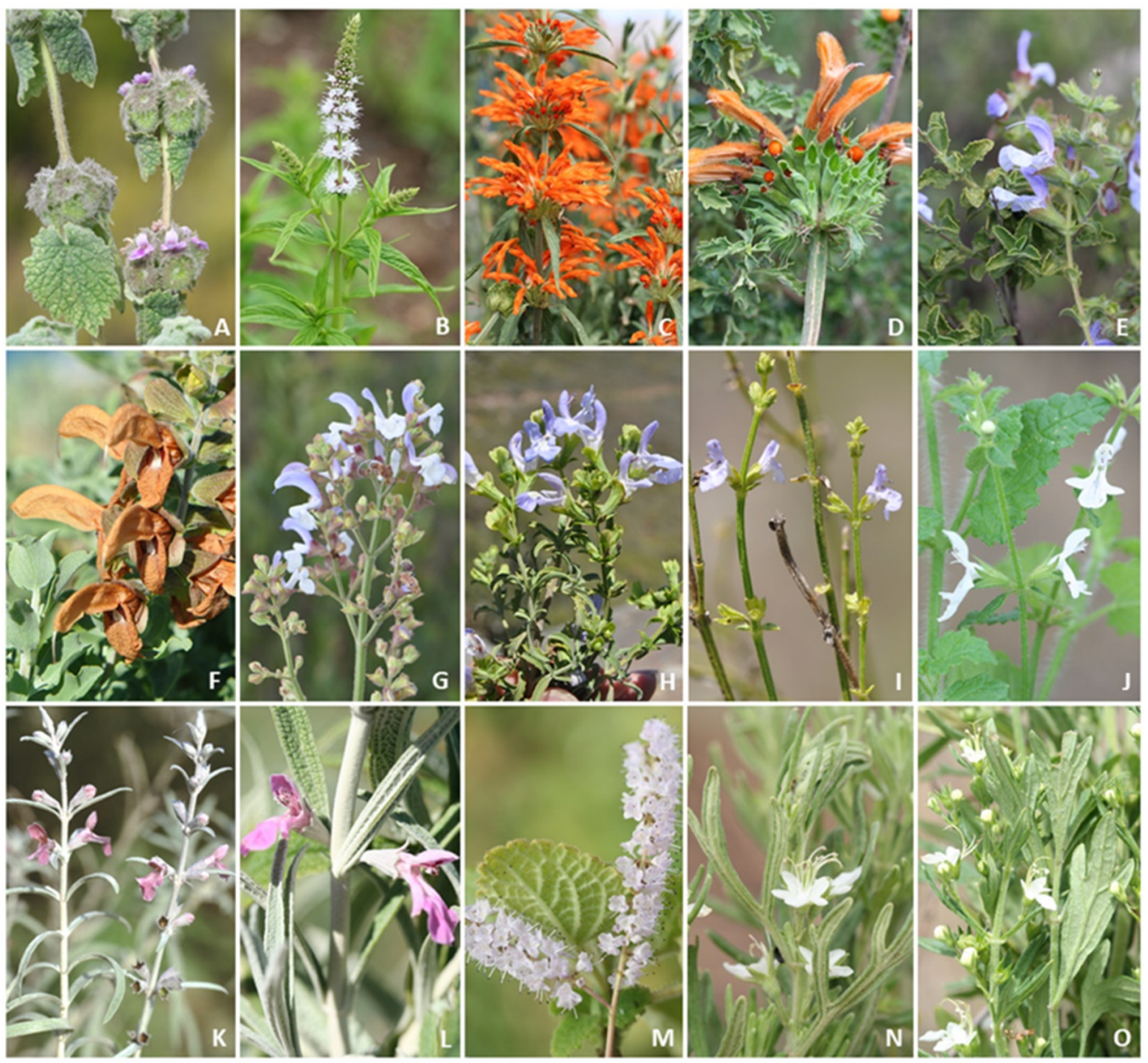

Figure 1. Southern African medicinal plants of the Lamiaceae that are commonly used in traditional medicine: (A) Pseudodictamnus africanus; (B) Mentha longifolia; (C) Leonotis leonurus; (D) L. ocymifolia; (E) Salvia africana; (F) S. aurea; (G) S. chamealaeagnea; (H) S. dentata; (I) S. stenophylla; (J) Stachys aethiopica; (K) S. linearis; (L) S. rugosa; (M) Tetradenia riparia; (N) Teucrium africanum and (O) T. trifidum. All photographs taken by B.-E. Van Wyk.

In this detailed review, we provide a broad overview of the botanical diversity, reported volatile and other chemical compounds and traditional uses of the Lamiaceae in southern Africa. The geographical area covered is the Flora of Southern Africa region 
(Figure 2), which includes South Africa, Botswana, Eswatini (Swaziland), Lesotho and Namibia. Our hypothesis was that the southern African Lamiaceae have remained poorly studied as a result of a lack of clarity on generic delimitations and phylogenetic relationships. The review therefore reflects on the current classification system of the family and provides a summary of the current state of knowledge regarding the secondary metabolites that have been reported in the literature, as well as traditional uses of southern African species (data from recent ethnobotanical surveys are included). The aim was to gain a more profound understanding of the patterns of chemical variation and ethnobotanical diversity, and to identify obvious knowledge gaps that require further study.

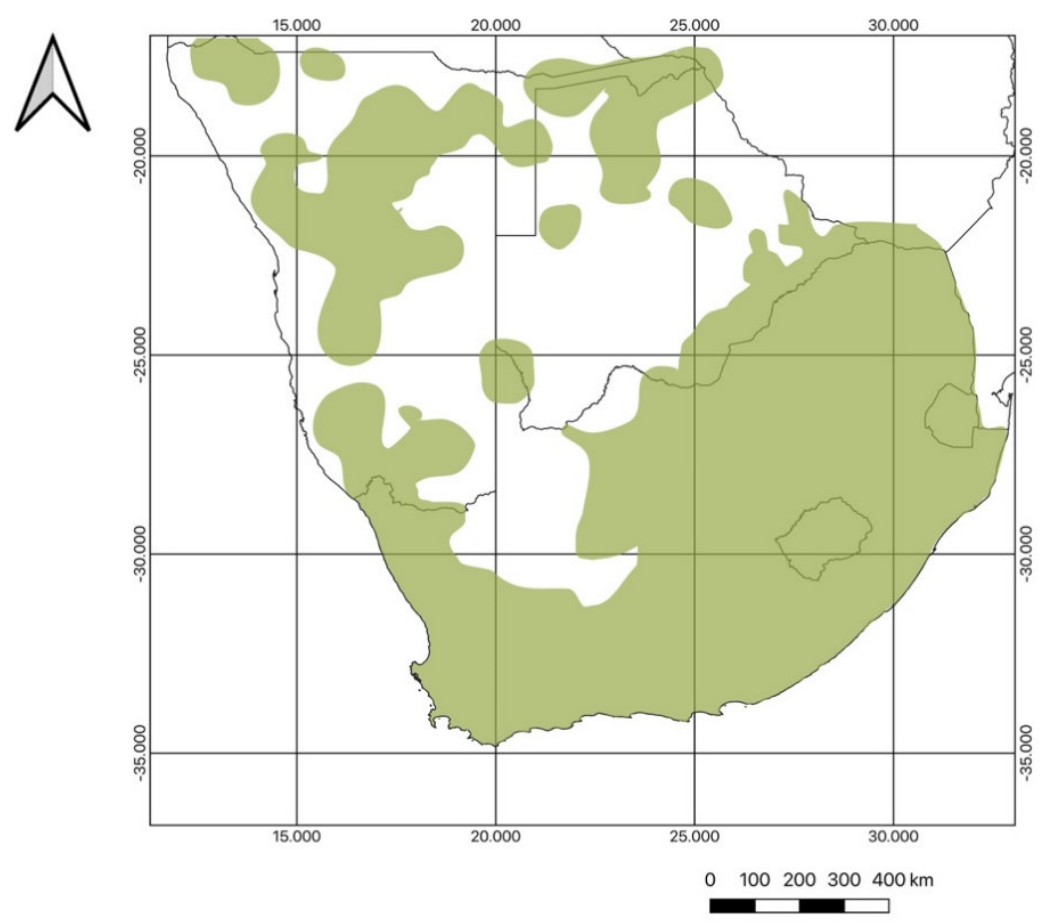

Figure 2. The geographical distribution of the Lamiaceae in southern Africa.

\section{Botanical Diversity}

Members of the Lamiaceae family are generally recognized by a combination of traits which include opposite leaves, bilaterally symmetric flowers with four stamens, and ovaries consisting of two fused carpels, each divided into two one-seeded chambers [16].

Southern African Lamiaceae comprises of 42 genera and 297 species (excluding subspecies and varieties; a full list of the 329 taxa can be found in Table S1) of which 105 species are endemic to the Flora of Southern Africa region (Figure 2), 171 are indigenous to the region and 21 are naturalized exotics (Figure 3). The five largest genera are Stachys (41 species), Syncolostemon E. Mey. ex Benth. (40 species), Coleus Lour. (34 species), Salvia (28 species) and Plectranthus (27 species). The smallest genera, comprising only a single species each, are: Ajuga L., Basilicum Moench, Cedronella Moench*, Haumaniastrum P.A. Duvign. and Plancke, Hoslundia Vahl, Kalaharia Baill., Karomia Dop, Lamium L., Marrubium L. *, Mesosphaerum P. Browne, Platostoma P. Beauv., Premna L., Prunella L. *, Pseudodictamnus africanus (L.) Salmaki and Siadati, Rabdosiella Codd, Satureja L. , Scutellaria*, and Volkameria P. Browne (naturalized exotics are indicated with an asterisk). 


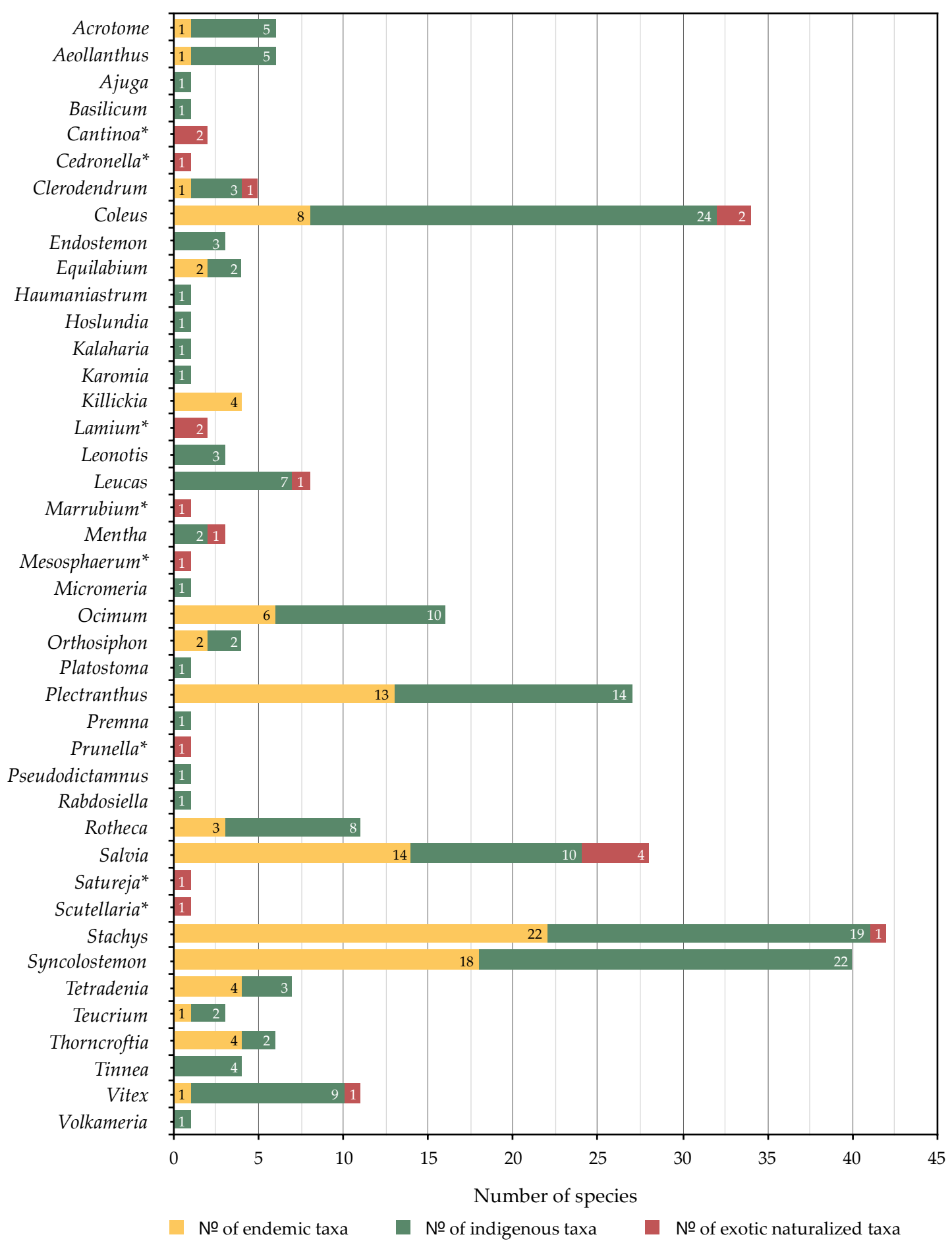

Figure 3. The genera and number of species (endemic, indigenous and naturalized) within southern African Lamiaceae.

The last taxonomic account of southern African Lamiaceae was by Codd in 1985 and included 232 species within 37 genera [17]. Since then, many studies have produced nomenclatural changes, resulting in the movement of one genus to another, or members from one family moved to another, based on molecular data. Several studies illustrate this, including the renaming of many Plectranthus species to Coleus [18], Becium Lindl. to Ocimum L. [19], Hemizygia (Benth.) Briq. to Syncolostemon [20], and members of other families (such as Verbenaceae J. St.-Hil.) being moved to Lamiaceae [21,22], thus making it difficult to find an up-to-date account of Lamiaceae species for the region. 
Similarly, the subfamilial and tribal classification of indigenous southern African Lamiaceae genera has only recently reached some stability because of an extensive molecular phylogenetic study [19]. The new classification system of 10 subfamilies provides, for the first time, a framework for future comparative studies to evaluate the chemotaxonomic value of secondary metabolites. The phylogenetic affinities of the indigenous southern African genera are shown in the following summary:

Subfamily 1. Prostantheroideae Luerssen. No southern African genera.

Subfamily 2. Symphorematoideae Briquet. No southern African genera.

Subfamily 3. Viticoideae Briquet. Vitex (ca. 250 spp., 10 in southern Africa).

Subfamily 4. Nepetoideae (Dumortier) Luerssen. This is the largest subfamily and contains ca. 118 genera and ca, 3400 species [22]. Rosmarinic acid is a potential synapomorphy for the subfamily. The tribe Mentheae Dumort. includes the genera Killickia Bräuchler, Heubl and Doroszenko, Mentha L., Micromeria Benth., Salvia, while the tribe Ocimeae Dumort. has two subtribes: Ociminae J.A. Schmidt, with the genera Basilicum, Endostemon N.E. Br., Haumaniastrum, Hoslundia, Ocimum, Orthosiphon Benth., Platostoma and Syncolostemon; subtribe Plectranthinae Endl., with the genera Aeollanthus C.Mart. ex Spreng., Coleus, Equilabium Mwany., A.J. Paton and Culham, Plectranthus, Rabdosiella, Tetradenia Benth. and Thorncroftia N.E. Br.

Subfamily 5. Premnoideae B. Li, R. G. Olmstead and P. D. Cantino. Premna (50-200 spp., only 1 in southern Africa).

Subfamily 6. Ajugoideae Kosteletzky. Ajuga, Clerodendrum L., Kalaharia, Karomia, Rotheca Raf., Teucrium and Volkameria.

Subfamily 7. Peronematoideae B. Li, R. G. Olmstead and P. D. Cantino. No southern African genera.

Subfamily 8. Scutellarioideae (Dumortier) Caruel. Tinnea Kotschy ex Hook. f. (19 species, all endemic to Africa, 4 in southern Africa).

Subfamily 9. Cymarioideae B. Li, R. G. Olmstead and P. D. Cantino. No southern African genera.

Subfamily 10. Lamioideae Harley. This is the largest subfamily in the Old World and is second only to Nepetoideae in terms of the numbers of taxa, with more than 60 genera and ca. 1200 species. It has been suggested [19] that allenic components in the seed oil may be a synapomorphy for the subfamily. Of the 10 tribes recognized [19], only three are represented in southern Africa: Stachydeae with Stachys, Marrubieae with Pseudodictamnus Fabr. and Leucadeae with Acrotome Benth. ex Endl., Harmsiella Briq., Leonotis (Pers.) R.Br. and Leucas R.Br.

\section{Chemical Compounds}

Several groups and sub-groups of variable circumscriptions exist in the Lamiaceae and as a result, the phytochemistry has been deemed complex by many researchers. The general idea is that there are two major groups within the family that produce secondary metabolites: those that produce volatile terpenoids (essential oil) (subfamily Nepetoideae), and those that produce non-volatile polar compounds and that are poor essential oil producers (subfamily Lamioideae) [23]. This generalization is no longer accurate, partly as a result of realignments of genera and partly because several members of the Lamioideae also produce essential oil. The essential oil producers are said to be highly aromatic and reported to possess diverse phytochemical profiles and as a result, exhibit a wide range of biological activities [24]. Major bioactive constituents such as volatile oil compounds (mostly mono- and sesquiterpenoids), flavonoids and hydroxycinnamic acids have been found in the most common Lamiaceae species which include those such as lemon balm, oregano, peppermint, rosemary, sage and thyme [25]. Some species are chemically highly variable and include several distinct chemotypes, producing varying amounts of major constituents within their volatile oils. Studies on Tunisian Mentha species revealed two types, one being pulegone dominated and the other menthone dominated; a study in Iran demonstrated piperitenone- and $\beta$-caryophyllene-dominated chemotypes. The essential 
oil composition of the common sage (Salvia officinalis L.) has been reported to vary from the "standard" $\alpha$-thujone > camphor $>1,8$-cineole chemotype, while studies on rosemary (Salvia rosmarinus Spenn.) from Saudi Arabia presented three chemotypes, a pattern which has similarly been reported from Algeria, Europe and India [26-28].

Non-volatile secondary metabolites, especially metabolites from polar fractions, can vary greatly at the species level. Generally, there are six main classes of secondary metabolites found in the Lamiaceae, namely: caffeoylquinic and other phenolic acids, flavonoids, iridoids, lignans, non-volatile terpenoids, and phenylethanoid glycosides [23]. One of the earliest accounts of phytochemical investigations of southern African Lamiaceae was in 1964 when marrubiin was isolated from Leonotis leonurus [29]. Since then, several compounds have been isolated and their chemical structures determined, primarily diterpenes [30-33], phenolic compounds [34], pyrones [35-37], and glycosides [38-40].

Of the 297 southern African Lamiaceae species, 101 have been chemically studied (i.e., essential oil analyses have been performed or phytochemical extractions and characterization have been made), equating to a total of $34 \%$ of species having been chemically profiled. Table 1 provides a summary of genera and species that have been studied and highlights the main compounds that have been recorded. The overall pattern for volatile compounds (Figure 4) shows the relatively small number of genera and species that have hitherto been studied. The same trend is seen in Figure 5 for eight classes of non-volatile secondary metabolites. Below we discuss the different classes of compounds in detail.

No of species where volatile oil has been extracted

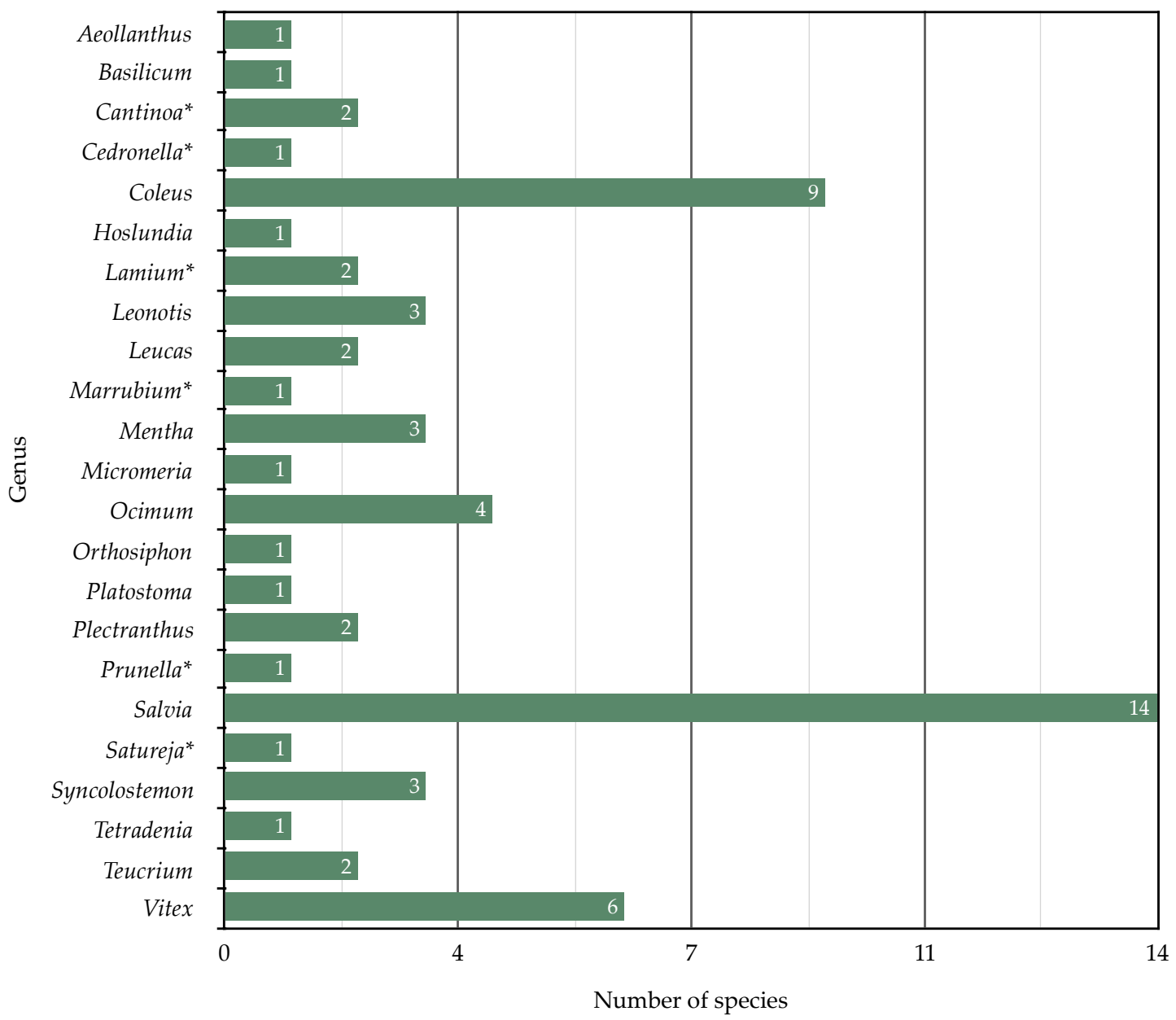

Figure 4. The numbers of species from 23 genera (out of 42) of southern African Lamiaceae that have been studied for their essential oil composition. 


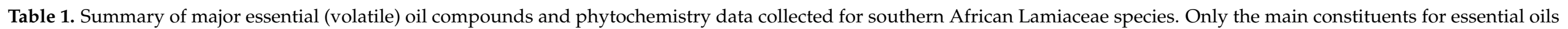
(compounds representing $>10 \%$ of total yield) and the main classes of compounds for phytochemical studies are listed.

\begin{tabular}{|c|c|c|c|c|c|c|c|c|c|}
\hline Genus & Species & $\begin{array}{l}\text { Main Compounds in } \\
\text { Essential Oil }\end{array}$ & $\begin{array}{l}\text { Number of } \\
\text { Samples } \\
\text { Analyzed }\end{array}$ & $\begin{array}{l}\text { Number of } \\
\text { Compounds } \\
\text { Reported }\end{array}$ & Reference & $\begin{array}{l}\text { Quality of } \\
\text { Study }\end{array}$ & $\begin{array}{l}\text { Other Phytochemical } \\
\text { Compounds }\end{array}$ & Reference & $\begin{array}{l}\text { Level of } \\
\text { Study }\end{array}$ \\
\hline \multirow[t]{2}{*}{ Acrotome } & \multirow[t]{2}{*}{ A. inflata } & \multirow[t]{2}{*}{-} & \multirow[t]{2}{*}{-} & \multirow[t]{2}{*}{-} & \multirow[t]{2}{*}{-} & \multirow[t]{2}{*}{-} & $\begin{array}{l}\text { Coumarins } \\
\text { Flavonoids } \\
\text { Tannins }\end{array}$ & {$[34]$} & $\star$ \\
\hline & & & & & & & Terpenoids & [41] & $\star$ \\
\hline \multirow{2}{*}{ Aeollanthus } & A. buchnerianus & - & - & - & - & - & Caffeic acid esters & [42] & $\star \star$ \\
\hline & A. parvifolius & $\alpha$-Muurolol & $1^{a}$ & 26 & [43] & $\star \star$ & - & - & - \\
\hline \multirow[b]{3}{*}{ Basilicum } & \multirow[b]{3}{*}{ B. polystachyon } & \multirow[b]{3}{*}{$\begin{array}{l}\text { Epiglobulol } \\
\text { Ylangene }\end{array}$} & \multirow[b]{3}{*}{$1^{\mathrm{a}}$} & \multirow[b]{3}{*}{64} & \multirow[b]{3}{*}[44]{} & \multirow[b]{3}{*}{$\star \star \star$} & Diterpenoids & [45] & $\star \star \star \star$ \\
\hline & & & & & & & Stachyonic acid A & [46] & 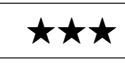 \\
\hline & & & & & & & $\begin{array}{l}\text { Alkaloids } \\
\text { Coumarins } \\
\text { Flavonoids } \\
\text { Phenols } \\
\text { Tannins } \\
\text { Triterpenes } \\
\text { Sterols } \\
\text { Saponins }\end{array}$ & [47] & $\star$ \\
\hline \multirow{4}{*}{ Cantinoa* } & \multirow{4}{*}{ C. americana * } & $\begin{array}{l}\alpha \text {-Bergamotene } \\
\beta \text {-Caryophyllene } \\
\text { Germacrene-A }\end{array}$ & $1^{b}$ & 30 & [48] & $\star \star$ & Spicigera Lactone & [49] & $\star \star$ \\
\hline & & \multirow{2}{*}{$\begin{array}{l}\delta \text {-3-Carene } \\
\beta \text {-Caryophyllene }\end{array}$} & \multirow{2}{*}{$1^{\mathrm{a}}$} & \multirow{2}{*}{34} & \multirow{2}{*}{ [50] } & \multirow{2}{*}{$\star \star$} & Labdane diterpenes & [51] & $\star \star \star$ \\
\hline & & & & & & & Alkaloids & [38] & $\star$ \\
\hline & & - & - & - & - & - & $\begin{array}{l}\text { Carbohydrates } \\
\text { Cardiac glycosides } \\
\text { Coumarins } \\
\text { Flavonoids }\end{array}$ & [38] & $\star$ \\
\hline
\end{tabular}


Table 1. Cont.

\begin{tabular}{|c|c|c|c|c|c|c|c|c|c|}
\hline Genus & Species & $\begin{array}{l}\text { Main Compounds in } \\
\text { Essential Oil }\end{array}$ & $\begin{array}{l}\text { Number of } \\
\text { Samples } \\
\text { Analyzed }\end{array}$ & $\begin{array}{l}\text { Number of } \\
\text { Compounds } \\
\text { Reported }\end{array}$ & Reference & $\begin{array}{l}\text { Quality of } \\
\text { Study }\end{array}$ & $\begin{array}{c}\text { Other Phytochemical } \\
\text { Compounds }\end{array}$ & Reference & $\begin{array}{l}\text { Level of } \\
\text { Study }\end{array}$ \\
\hline \multirow{4}{*}{$\begin{array}{l}\text { Cantinoa* } \\
\text { (Cont.) }\end{array}$} & $\begin{array}{l}\text { C. americana* } \\
\text { (Cont.) }\end{array}$ & - & - & - & - & - & $\begin{array}{l}\text { Quinones } \\
\text { Resins } \\
\text { Saponins } \\
\text { Steroids } \\
\text { Terpenoids } \\
\text { Vitannins }\end{array}$ & [38] & $\star$ \\
\hline & \multirow{3}{*}{ C. mutabilis * } & $\begin{array}{l}\text { Bicyclogermacrene } \\
\beta \text {-Caryophyllene } \\
\text { Curzerene } \\
\text { Germacrene-D }\end{array}$ & $2^{c}$ & 24 & [52] & $\star \star$ & \multirow{3}{*}{ Triterpenoids } & \multirow{3}{*}[53]{} & \multirow{3}{*}{$\star \star \star$} \\
\hline & & $\begin{array}{l}\text { 1,8-Cineole } \\
\text { Limonene } \\
\text { Spathulenol }\end{array}$ & $12^{\#}$ & 105 & [54] & $\star \star \star$ & & & \\
\hline & & $\begin{array}{l}\text { Camphor } \\
\text { cis-Dihydrocarvone } \\
\text { trans-Dihydrocarvone }\end{array}$ & $1^{\mathrm{a}}$ & 31 & [55] & $\star \star$ & & & \\
\hline \multirow{2}{*}{ Cedronella * } & \multirow{2}{*}{ C. canariensis * } & $\begin{array}{l}\beta \text {-Pinene } \\
\text { Pinocarvone }\end{array}$ & $2^{a}$ & 27 & [56] & \multirow{2}{*}{ 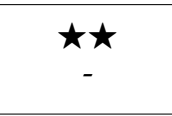 } & Terpenoids & {$[57]$} & $\star \star \star \star$ \\
\hline & & p-Allyl anisole & - & - & - & & Caffeic acid esters & {$[42]$} & $\star \star$ \\
\hline \multirow[b]{2}{*}{ Coleus } & C. aliciae & - & - & - & - & - & Diterpenoids & [58] & $\#$ \\
\hline & C. amboinicus & $\begin{array}{l}\text { Carvacrol } \\
p \text {-Cymene } \\
\gamma \text {-Terpinene }\end{array}$ & $2^{b}$ & 12 & [59] & $\star \star$ & Phenolics & {$[60]$} & $\star \star$ \\
\hline
\end{tabular}


Table 1. Cont.

\begin{tabular}{|c|c|c|c|c|c|c|c|c|c|}
\hline Genus & Species & $\begin{array}{l}\text { Main Compounds in } \\
\text { Essential Oil ^ }\end{array}$ & $\begin{array}{l}\text { Number of } \\
\text { Samples } \\
\text { Analyzed }\end{array}$ & $\begin{array}{c}\text { Number of } \\
\text { Compounds } \\
\text { Reported }\end{array}$ & Reference & $\begin{array}{l}\text { Quality of } \\
\text { Study }\end{array}$ & $\begin{array}{l}\text { Other Phytochemical } \\
\text { Compounds }\end{array}$ & Reference & $\begin{array}{l}\text { Level of } \\
\text { Study }\end{array}$ \\
\hline \multirow{15}{*}{$\begin{array}{l}\text { Coleus } \\
\text { (Cont.) }\end{array}$} & \multirow{6}{*}{ C. amboinicus (Cont.) } & $\begin{array}{l}\text { Carvacrol } \\
\text { Caryophyllene } \\
\alpha \text {-Bergamotene } \\
\end{array}$ & $1^{b}$ & 9 & {$[61]$} & $\star \star$ & Terpenoids & [31] & $\star \star \star$ \\
\hline & & $\begin{array}{l}\text { (E)-Caryophyllene } \\
\text { Germacrene-D } \\
\text { Zingiberene }\end{array}$ & $1^{\mathrm{a}}$ & 8 & {$[62]$} & $\star \star$ & Flavonoids & [63] & $\star \star$ \\
\hline & & $\begin{array}{l}\text { Carvacrol } \\
\text { Caryophyllene } \\
\alpha \text {-Bergamotene }\end{array}$ & $8^{a}$ & 32 & {$[64]$} & $\star \star$ & Flavonoids & [65] & $\star$ \\
\hline & & $\begin{array}{l}\text { Carvacrol } \\
\text { Caryophyllene } \\
\beta \text {-Cymene } \\
\gamma \text {-Terpinene }\end{array}$ & $1^{a}$ & 27 & {$[66]$} & $\star \star$ & \multirow{3}{*}{-} & \multirow{3}{*}{-} & \multirow{3}{*}{-} \\
\hline & & $\begin{array}{l}\text { Carvacrol } \\
\beta \text {-Caryophyllene } \\
\gamma \text {-Terpinene }\end{array}$ & $1^{\mathrm{a}}$ & 43 & {$[67]$} & $\star \star \star$ & & & \\
\hline & & Carvacrol & $1^{\mathrm{a}}$ & 13 & [68] & $\star \star$ & & & \\
\hline & \multirow{9}{*}{ C. barbatus * } & \multirow{9}{*}{$\begin{array}{l}\text { Caryophyllene } \\
\beta \text {-Phellandrene } \\
\beta \text {-Linalool }\end{array}$} & \multirow{9}{*}{$1^{\mathrm{a}, \mathrm{b}}$} & \multirow{9}{*}{137} & \multirow{9}{*}{ [69] } & \multirow{9}{*}{$\star \star \star$} & Diterpenoids & [58] & $\#$ \\
\hline & & & & & & & Diterpenoids & {$[70]$} & $\#$ \\
\hline & & & & & & & Phenolics & [71] & $\star \star$ \\
\hline & & & & & & & Diterpenoids & [72] & $\star \star$ \\
\hline & & & & & & & Diterpenoids & [73] & $\star \star$ \\
\hline & & & & & & & Diterpenoids & [74] & $\star \star$ \\
\hline & & & & & & & Diterpenoids & [75] & $\star \star$ \\
\hline & & & & & & & Diterpenoids & [76] & $\star \star$ \\
\hline & & & & & & & Diterpenoids & [77] & $\star \star$ \\
\hline
\end{tabular}


Table 1. Cont.

\begin{tabular}{|c|c|c|c|c|c|c|c|c|c|}
\hline Genus & Species & $\begin{array}{l}\text { Main Compounds in } \\
\text { Essential Oil }\end{array}$ & $\begin{array}{l}\text { Number of } \\
\text { Samples } \\
\text { Analyzed }\end{array}$ & $\begin{array}{l}\text { Number of } \\
\text { Compounds } \\
\text { Reported }\end{array}$ & Reference & $\begin{array}{l}\text { Quality of } \\
\text { Study }\end{array}$ & $\begin{array}{l}\text { Other Phytochemical } \\
\text { Compounds }\end{array}$ & Reference & $\begin{array}{l}\text { Level of } \\
\text { Study }\end{array}$ \\
\hline \multirow{15}{*}{$\begin{array}{l}\text { Coleus } \\
\text { (Cont.) }\end{array}$} & \multirow{3}{*}{ C. caninus } & \multirow{3}{*}{$\begin{array}{l}\beta \text {-Caryophyllene } \\
\beta \text {-Pinene } \\
\text { Terpinyl acetate }\end{array}$} & \multirow{3}{*}{$2^{a, b}$} & \multirow{3}{*}{131} & \multirow{3}{*}{ [69] } & \multirow{3}{*}{$\star \star \star$} & $\begin{array}{l}\text { Phenylpropanoids } \\
\text { Terpenoids }\end{array}$ & [78] & $\star \star \star$ \\
\hline & & & & & & & Caffeic acid esters & [79] & $\star \star$ \\
\hline & & & & & & & Caffeic acid esters & [42] & $\star \star$ \\
\hline & \multirow{5}{*}{ C. comosus * } & $\begin{array}{l}\alpha \text {-Pinene } \\
\text { Sabinene } \\
\beta \text {-Pinene }\end{array}$ & $3^{b, d}$ & 77 & [80] & $\star \star \star$ & Diterpenoids & [81] & $\star \star \star$ \\
\hline & & $\begin{array}{l}\alpha \text {-Thujene } \\
\beta \text {-Caryophyllene }\end{array}$ & $11^{\mathrm{b}}$ & 33 & [82] & $\star \star$ & Carboxylic acid & [83] & $\star \star \star$ \\
\hline & & \multirow{3}{*}{-} & \multirow{3}{*}{-} & \multirow{3}{*}{-} & \multirow{3}{*}{-} & \multirow{3}{*}{-} & Diterpenoids & [84] & $\star \star \star \star$ \\
\hline & & & & & & & Phenolic acids & [85] & $\star \star$ \\
\hline & & & & & & & Diterpenoids & [86] & $\star \star \star$ \\
\hline & \multirow[t]{2}{*}{ C. cylindraceus } & \multirow[t]{2}{*}{$\begin{array}{l}\alpha \text {-Thujene } \\
\beta \text {-Maaliene }\end{array}$} & \multirow[t]{2}{*}{$1^{a}$} & \multirow[t]{2}{*}{23} & \multirow[t]{2}{*}[88]{} & \multirow[t]{2}{*}{$\star \star$} & $\begin{array}{l}\text { Triterpene ester } \\
\text { Steroids }\end{array}$ & [89] & $\star \star$ \\
\hline & & & & & & & Flavonoids & [90] & $\star \star$ \\
\hline & \multirow{5}{*}{ C. grandidentatus } & \multirow{3}{*}{$\begin{array}{l}\text { Camphor } \\
\tau \text {-Cadinol }\end{array}$} & \multirow{3}{*}{$1^{a}$} & \multirow{3}{*}{62} & \multirow{3}{*}{ [91] } & \multirow{3}{*}{$\star \star \star$} & Diterpenoids & {$[32]$} & $\star \star$ \\
\hline & & & & & & & Diterpenoids & [92] & $\star \star \star$ \\
\hline & & & & & & & Diterpenoids & [87] & $\star \star \star$ \\
\hline & & Methyl eugenol & $1^{\mathrm{a}}$ & 22 & [93] & $\star \star$ & Terpenoids & [94] & $\star \star \star$ \\
\hline & & Pipertone oxide & $1^{a}$ & 25 & [95] & $\star \star$ & Diterpenoids & [96] & $\star \star$ \\
\hline
\end{tabular}


Table 1. Cont.

\begin{tabular}{|c|c|c|c|c|c|c|c|c|c|}
\hline Genus & Species & $\begin{array}{l}\text { Main Compounds in } \\
\text { Essential Oil }\end{array}$ & $\begin{array}{l}\text { Number of } \\
\text { Samples } \\
\text { Analyzed }\end{array}$ & $\begin{array}{l}\text { Number of } \\
\text { Compounds } \\
\text { Reported }\end{array}$ & Reference & $\begin{array}{l}\text { Quality of } \\
\text { Study }\end{array}$ & $\begin{array}{l}\text { Other Phytochemical } \\
\text { Compounds }\end{array}$ & Reference & $\begin{array}{l}\text { Level of } \\
\text { Study }\end{array}$ \\
\hline & \multirow{3}{*}{ C. hadiensis } & \multirow{3}{*}{-} & \multirow{3}{*}{-} & \multirow{3}{*}{-} & \multirow{3}{*}{-} & \multirow{3}{*}{-} & Diterpenoids & [97] & $\star \star$ \\
\hline & & & & & & & $\begin{array}{l}\text { Coumarins } \\
\text { Flavonoids } \\
\text { Tannins } \\
\text { Terpenes } \\
\text { Sterols }\end{array}$ & [93] & $\star$ \\
\hline & & & & & & & Flavonoids & [90] & $\star \star$ \\
\hline \multirow{8}{*}{$\begin{array}{l}\text { Coleus } \\
\text { (Cont.) }\end{array}$} & \multirow{3}{*}{ C. hereroensis } & \multirow{3}{*}{-} & \multirow{3}{*}{-} & \multirow{3}{*}{-} & \multirow{3}{*}{-} & \multirow{3}{*}{-} & Diterpenoids & [98] & $\star \star$ \\
\hline & & & & & & & Diterpenoids & [99] & $\star \star$ \\
\hline & & & & & & & $\begin{array}{l}\text { Aristolane } \\
\text { sesquiterpene aldehyde }\end{array}$ & [100] & $\star \star$ \\
\hline & \multirow{2}{*}{ C. madagascariensis } & \multirow{2}{*}{$\begin{array}{l}\alpha \text {-Fenchyl acetate } \\
\beta \text {-Caryophyllene }\end{array}$} & \multirow{2}{*}{$3^{d}$} & \multirow{2}{*}{23} & \multirow{2}{*}{ [101] } & \multirow{2}{*}{$\star \star$} & $\begin{array}{l}\text { Diterpenoids } \\
\text { Phenolic acid }\end{array}$ & [102] & $\star \star$ \\
\hline & & & & & & & $\begin{array}{l}\text { Diterpenoids } \\
\text { Phenolic acid }\end{array}$ & [103] & $\star \star$ \\
\hline & \multirow{3}{*}{ C. neochilus } & $\begin{array}{l}\text { Caryophyllene oxide } \\
\beta \text {-Caryophyllene } \\
\alpha \text {-Pinene }\end{array}$ & $1^{\mathrm{a}}$ & 31 & [104] & $\star \star$ & $\begin{array}{l}\text { Flavonoid glycosides } \\
\text { Polyphenols }\end{array}$ & [39] & $\star \star \star$ \\
\hline & & $\begin{array}{l}\text { Aromadendrene } \\
\text { Selina-3,7(11)-diene }\end{array}$ & $1^{\mathrm{a}}$ & 80 & [105] & $\star \star$ & Phenolic acids & [106] & $\#$ \\
\hline & & $\begin{array}{l}\beta \text {-Caryophyllene } \\
\alpha \text {-Pinene } \\
\alpha \text {-Thujene }\end{array}$ & $1^{\mathrm{a}}$ & 17 & [107] & $\star \star$ & - & - & - \\
\hline
\end{tabular}


Table 1. Cont.

\begin{tabular}{|c|c|c|c|c|c|c|c|c|c|}
\hline Genus & Species & $\begin{array}{l}\text { Main Compounds in } \\
\text { Essential Oil ^ }\end{array}$ & $\begin{array}{c}\text { Number of } \\
\text { Samples } \\
\text { Analyzed }\end{array}$ & $\begin{array}{c}\text { Number of } \\
\text { Compounds } \\
\text { Reported }\end{array}$ & Reference & $\begin{array}{l}\text { Quality of } \\
\text { Study }\end{array}$ & $\begin{array}{l}\text { Other Phytochemical } \\
\text { Compounds }\end{array}$ & Reference & $\begin{array}{l}\text { Level of } \\
\text { Study }\end{array}$ \\
\hline & \multirow[t]{2}{*}{ C. porcatus } & \multirow[t]{2}{*}{-} & \multirow[t]{2}{*}{-} & \multirow[t]{2}{*}{-} & \multirow[t]{2}{*}{-} & \multirow[t]{2}{*}{-} & $\begin{array}{l}\text { Diterpenoids } \\
\text { Phenolic acid }\end{array}$ & [108] & $\star \star$ \\
\hline & & & & & & & Diterpenoids & [109] & $\star \star \star$ \\
\hline & C. rotundifolius & - & - & - & - & - & $\begin{array}{l}\text { Alcohols } \\
\text { Aldehydes } \\
\text { Alkanes } \\
\text { Alkyne } \\
\text { Amines } \\
\text { Aromatics } \\
\text { Carboxylic acid } \\
\text { Chloro compounds }\end{array}$ & [110] & $\star \star \star$ \\
\hline \multirow[t]{2}{*}{$\begin{array}{l}\text { Coleus } \\
\text { (Cont.) }\end{array}$} & $\begin{array}{l}\text { C. rotundifolius } \\
\text { (Cont.) }\end{array}$ & - & - & - & - & - & $\begin{array}{l}\text { Isocynate } \\
\text { Isocyanides } \\
\text { Ketones } \\
\text { Phenols } \\
\text { Primary alcohols } \\
\text { Tertiary alcohols }\end{array}$ & [110] & $\star \star \star$ \\
\hline & C. venteri & - & - & - & - & - & Acetophenones & [111] & $\star \star$ \\
\hline Endostemon & E. obtusifolius & - & - & - & - & - & Caffeic acid esters & {$[42]$} & $\star \star$ \\
\hline Equilabium & E. petiolare & - & - & - & - & - & Flavonoids & {$[90]$} & $\star \star$ \\
\hline \multirow{4}{*}{ Hoslundia } & \multirow{4}{*}{ H. opposita } & \multirow{2}{*}{ Eugenol } & \multirow{2}{*}{$5^{d}$} & \multirow{2}{*}{88} & \multirow{2}{*}{ [112] } & \multirow{2}{*}{$\star \star \star$} & Abietane-type esters & [113] & $\star \star \star$ \\
\hline & & & & & & & Flavonoids & [114] & $\star \star$ \\
\hline & & \multirow{2}{*}{$\begin{array}{l}\text { Camphor } \\
\text { 1,8-Cineole }\end{array}$} & \multirow{2}{*}{$1^{\mathrm{a}}$} & \multirow{2}{*}{37} & \multirow{2}{*}{ [115] } & \multirow{2}{*}{$\star \star$} & Flavonoids & [116] & $\star \star$ \\
\hline & & & & & & & Flavonoids & [117] & $\star \star$ \\
\hline
\end{tabular}


Table 1. Cont.

\begin{tabular}{|c|c|c|c|c|c|c|c|c|c|}
\hline Genus & Species & $\begin{array}{l}\text { Main Compounds in } \\
\text { Essential Oil }\end{array}$ & $\begin{array}{c}\text { Number of } \\
\text { Samples } \\
\text { Analyzed }\end{array}$ & $\begin{array}{l}\text { Number of } \\
\text { Compounds } \\
\text { Reported }\end{array}$ & Reference & $\begin{array}{l}\text { Quality of } \\
\text { Study }\end{array}$ & $\begin{array}{c}\text { Other Phytochemical } \\
\text { Compounds }\end{array}$ & Reference & $\begin{array}{l}\text { Level of } \\
\text { Study }\end{array}$ \\
\hline \multirow{8}{*}{ Lamium * } & \multirow{6}{*}{ L. amplexicaule* } & - & $1^{\mathrm{a}}$ & 33 & [118] & $\star \star$ & Iridoid glucosides & [119] & $\#$ \\
\hline & & $\begin{array}{l}\text { Camphor } \\
\text { Germacrene-D }\end{array}$ & $1^{\mathrm{a}}$ & 48 & [120] & $\star \star$ & Iridoid glucosides & [121] & $\star \star \star$ \\
\hline & & \multirow{4}{*}{-} & \multirow{4}{*}{ - } & \multirow{4}{*}{-} & \multirow{4}{*}{ - } & \multirow{4}{*}{-} & Iridoid glucosides & [122] & $\star \star \star$ \\
\hline & & & & & & & Terpenoids & [123] & $\star \star$ \\
\hline & & & & & & & Iridoid glucosides & [124] & $\star \star$ \\
\hline & & & & & & & Terpenoids & [125] & $\star \star \star$ \\
\hline & \multirow[t]{2}{*}{ L. galeobdolon* } & \multirow[t]{2}{*}{-} & \multirow{2}{*}{$1^{\mathrm{a}}$} & \multirow{2}{*}{21} & \multirow[t]{2}{*}{ [126] } & \multirow{2}{*}{$\star \star$} & $\begin{array}{l}\text { Benzoxazinoids } \\
\text { Benzoxazinones }\end{array}$ & [122] & $\star \star \star$ \\
\hline & & & & & & & Iridoid glucosides & [40] & $\star \star$ \\
\hline \multirow{4}{*}{ Leonotis } & \multirow{4}{*}{ L. leonurus } & $\begin{array}{l}\text { Bourbonene } \\
\text { cis- } \beta \text {-Ocimene } \\
\text { Germacrene-D } \\
\text { Limonene } \\
\alpha \text {-Humulene } \\
\beta \text {-Caryophyllene }\end{array}$ & $3^{d}$ & 25 & [127] & $\star \star$ & Diterpenes & [128] & $\star \star$ \\
\hline & & $\begin{array}{l}\text { Germacrene-D } \\
\text { Limonene } \\
\beta \text {-Caryophyllene }\end{array}$ & $1^{\mathrm{a}}$ & 30 & [129] & $\star \star$ & Diterpenes & [130] & $\star \star$ \\
\hline & & $\begin{array}{l}\beta \text {-Caryophyllene } \\
\text { Germacrene-D }\end{array}$ & $1^{\mathrm{a}}$ & 56 & [131] & $\star \star \star$ & Diterpenes & [132] & $\star \star \star$ \\
\hline & & $\begin{array}{l}\alpha \text {-Pinene } \\
\beta \text {-Caryophyllene }\end{array}$ & $1^{\mathrm{a}}$ & 33 & [133] & $\star \star$ & $\begin{array}{l}\text { Alkaloids } \\
\text { Dicarboxylic acid } \\
\text { Diterpene esters } \\
\text { Flavonoids } \\
\text { Iridoid glycoside }\end{array}$ & [10] & $\#$ \\
\hline
\end{tabular}


Table 1. Cont.

\begin{tabular}{|c|c|c|c|c|c|c|c|c|c|}
\hline Genus & Species & $\begin{array}{l}\text { Main Compounds in } \\
\text { Essential Oil }\end{array}$ & $\begin{array}{l}\text { Number of } \\
\text { Samples } \\
\text { Analyzed }\end{array}$ & $\begin{array}{l}\text { Number of } \\
\text { Compounds } \\
\text { Reported }\end{array}$ & Reference & $\begin{array}{l}\text { Quality of } \\
\text { Study }\end{array}$ & $\begin{array}{c}\text { Other Phytochemical } \\
\text { Compounds }\end{array}$ & Reference & $\begin{array}{c}\text { Level of } \\
\text { Study }\end{array}$ \\
\hline & & \multirow{9}{*}{$\begin{array}{l}\text { Caryophyllene } \\
\text { Germacrene-D }\end{array}$} & \multirow{9}{*}{$1^{\mathrm{a}}$} & \multirow{9}{*}{21} & \multirow{9}{*}{ [134] } & \multirow{9}{*}{$\star \star$} & Diterpene ester & [135] & $\star \star \star \star$ \\
\hline & & & & & & & Unknown compounds & [136] & $\star$ \\
\hline & & & & & & & Flavonoids & [137] & $\star \star \star$ \\
\hline & & & & & & & Labdane diterpenoids & [138] & $\star$ \\
\hline & & & & & & & $\begin{array}{l}\text { Flavonoids } \\
\text { Labdane diterpenoids } \\
\text { Phenolics }\end{array}$ & [140] & $\#$ \\
\hline & & & & & & & Labdane diterpenoids & [141] & $\star \star \star$ \\
\hline & & & & & & & Labdane diterpenoids & [142] & $\star$ \\
\hline & & & & & & & Diterpenoids & [143] & $\star \star \star$ \\
\hline & & & & & & & Labdane diterpenoid & [29] & $\star$ \\
\hline \multirow{6}{*}{$\begin{array}{l}\text { Leonotis } \\
\text { (Cont.) }\end{array}$} & \multirow{3}{*}{ L. nepetifolia } & E-Ocimene & $2^{c}$ & 31 & [145] & $\star \star$ & Diterpenoids & [146] & $\star \star \star$ \\
\hline & & $\begin{array}{l}\beta \text {-Caryophyllene } \\
\text { Germacrene-D } \\
\delta \text {-Selinene }\end{array}$ & $1^{\mathrm{a}}$ & 43 & [147] & $\star \star$ & $\begin{array}{l}\text { Carotenoids } \\
\text { Flavonoids } \\
\text { Phenolics }\end{array}$ & [148] & $\star \star$ \\
\hline & & Germacrene-D & - & 3 & [134] & $\star \star$ & Diterpenoids & [149] & $\star \star$ \\
\hline & \multirow{3}{*}{ L. ocymifolia } & Caryophyllene oxide & $1^{\mathrm{a}}$ & 68 & [150] & $\star \star \star$ & \multirow{3}{*}{ Diterpenoids } & \multirow{3}{*}[151]{} & \multirow{3}{*}{$\star \star$} \\
\hline & & $\begin{array}{l}\text { Caryophyllene } \\
\text { Germacrene }\end{array}$ & $1^{\mathrm{a}}$ & 21 & [134] & $\star \star$ & & & \\
\hline & & $\begin{array}{l}(Z)-\beta \text {-Ocimene } \\
\beta \text {-Caryophyllene } \\
\text { Germacrene-D }\end{array}$ & $1^{\mathrm{a}}$ & 26 & [129] & $\star \star$ & & & \\
\hline
\end{tabular}


Table 1. Cont.

\begin{tabular}{|c|c|c|c|c|c|c|c|c|c|}
\hline Genus & Species & $\begin{array}{l}\text { Main Compounds in } \\
\text { Essential Oil }\end{array}$ & $\begin{array}{c}\text { Number of } \\
\text { Samples } \\
\text { Analyzed }\end{array}$ & $\begin{array}{c}\text { Number of } \\
\text { Compounds } \\
\text { Reported }\end{array}$ & Reference & $\begin{array}{l}\text { Quality of } \\
\text { Study }\end{array}$ & $\begin{array}{l}\text { Other Phytochemical } \\
\text { Compounds }\end{array}$ & Reference & $\begin{array}{l}\text { Level of } \\
\text { Study }\end{array}$ \\
\hline & $\begin{array}{l}\text { L. ocymifolia var. } \\
\text { ocymifolia }\end{array}$ & $\begin{array}{l}\text { Caryophyllene } \\
\text { Germacrene-D }\end{array}$ & $1^{\mathrm{a}}$ & 10 & [134] & $\star \star$ & - & - & \\
\hline & $\begin{array}{l}\text { L. ocymifolia var. } \\
\text { raineriana }\end{array}$ & Germacrene-D & $1^{\mathrm{a}}$ & 27 & [152] & $\star \star$ & Diterpenoids & [153] & $\star \star$ \\
\hline & $\begin{array}{l}\text { L. ocymifolia var. } \\
\text { schinzii }\end{array}$ & $\begin{array}{l}\text { Caryophyllene } \\
\text { Germacrene-D }\end{array}$ & $1^{\mathrm{a}}$ & 16 & [134] & $\star \star$ & - & - & \\
\hline \multirow{3}{*}{ Leucas } & L. capensis & - & - & - & - & - & Diterpenoids & [154] & $\star \star \star$ \\
\hline & L. glabrata & $\begin{array}{l}\text { Isomenthone } \\
\text { Piperitone } \\
\text { Pulegone }\end{array}$ & $1^{\mathrm{a}}$ & 37 & [155] & $\star \star$ & - & - & - \\
\hline & L. martinicensis & Germacrene-D & $1^{\mathrm{a}}$ & 39 & [147] & $\star \star$ & $\begin{array}{l}\text { Alkaloids } \\
\text { Flavonoids } \\
\text { Glycosides } \\
\text { Saponins }\end{array}$ & [156] & $\star$ \\
\hline Marrubium * & M. vulgare* & $\gamma$-Eudesmol & $1^{b}$ & 34 & [159] & $\star \star$ & Flavonoids & [160] & $\star \star \star$ \\
\hline \multirow[b]{3}{*}{$\begin{array}{l}\text { Marrubium * } \\
\text { (Cont.) }\end{array}$} & \multirow[b]{3}{*}{ M. vulgare* (Cont.) } & - & - & - & - & - & $\begin{array}{l}\text { Phenylethanoid } \\
\text { Terpenoids }\end{array}$ & [160] & $\star \star \star$ \\
\hline & & $\begin{array}{l}\beta \text {-Caryophyllene } \\
\text { Germacrene-D }\end{array}$ & $1^{\mathrm{a}}$ & 12 & [161] & $\star \star$ & Diterpenoids & [162] & $\star \star \star \star$ \\
\hline & & $\begin{array}{l}\beta \text {-Caryophyllene } \\
\beta \text {-Bisabolene }\end{array}$ & $1^{\mathrm{a}}$ & 33 & [163] & $\star \star$ & $\begin{array}{l}\text { Coumarins } \\
\text { Flavonoids } \\
\text { Phenolic acids } \\
\text { Phenylpropanoid acids } \\
\text { Phenylpropanoid esters } \\
\text { Phenylpropanoid } \\
\text { glycosides } \\
\text { Terpenoids }\end{array}$ & [164] & $\#$ \\
\hline
\end{tabular}


Table 1. Cont.

\begin{tabular}{|c|c|c|c|c|c|c|c|c|c|}
\hline Genus & Species & $\begin{array}{l}\text { Main Compounds in } \\
\text { Essential Oil }\end{array}$ & $\begin{array}{c}\text { Number of } \\
\text { Samples } \\
\text { Analyzed }\end{array}$ & $\begin{array}{l}\text { Number of } \\
\text { Compounds } \\
\text { Reported }\end{array}$ & Reference & $\begin{array}{l}\text { Quality of } \\
\text { Study }\end{array}$ & $\begin{array}{l}\text { Other Phytochemical } \\
\text { Compounds }\end{array}$ & Reference & $\begin{array}{c}\text { Level of } \\
\text { Study }\end{array}$ \\
\hline \multirow{11}{*}{ Mentha } & \multirow{7}{*}{ M. aquatica } & $\begin{array}{l}\text { Linalool } \\
\text { Linalyl acetate } \\
\alpha \text {-Pinene }\end{array}$ & $1^{\mathrm{a}}$ & 42 & [165] & $\star \star$ & Phenolics & [166] & $\star \star$ \\
\hline & & $\begin{array}{l}\text { L-Menthone } \\
\text { Pulegone }\end{array}$ & $1^{a}$ & 18 & [26] & $\star \star$ & Phenolics & [167] & $\star \star$ \\
\hline & & 1,8-Cineole & $1^{\mathrm{a}}$ & 31 & [169] & $\star \star$ & Diterpenes & [170] & $\star \star$ \\
\hline & & $\begin{array}{l}\text { Limonene } \\
\beta \text {-Caryophyllene } \\
\text { Germacrene-D }\end{array}$ & $1^{\mathrm{b}}$ & 34 & {$[171]$} & $\star \star$ & Flavonoids & {$[172]$} & $\star \star$ \\
\hline & & $\begin{array}{l}\text { 1,8-Cineole } \\
\text { Methanofuran } \\
\beta \text {-Caryophyllene }\end{array}$ & $1^{\mathrm{a}}$ & 29 & {$[173]$} & $\star \star$ & Flavonoids & [174] & $\star \star$ \\
\hline & & \multirow{2}{*}{-} & \multirow{2}{*}{-} & \multirow{2}{*}{-} & \multirow{2}{*}{-} & \multirow{2}{*}{-} & Flavonoid glycones & [175] & $\star \star \star \star$ \\
\hline & & & & & & & Monoterpene ketones & [176] & $\star \star \star$ \\
\hline & M. longifolia & $\begin{array}{l}\text { Isomenthone } \\
\text { Pulegone }\end{array}$ & $1^{\mathrm{a}}$ & 36 & [177] & $\star \star$ & Flavonoids & [178] & $\star \star \star$ \\
\hline & \multirow{3}{*}{ M. longifolia (Cont.) } & $\begin{array}{l}\text { Carvone } \\
\text { Limonene }\end{array}$ & $1^{\mathrm{a}}$ & 23 & [179] & $\star \star$ & Flavonoids & [180] & $\star \star \star$ \\
\hline & & $\begin{array}{l}\text { 1,8-Cineole } \\
\text { Menthone } \\
\text { Pulegone }\end{array}$ & $4^{\mathrm{d}}$ & 34 & [181] & $\star \star$ & Flavonoids & [182] & $\star \star \star$ \\
\hline & & $\begin{array}{l}\text { 1,8-Cineole } \\
\text { Isomenthone } \\
\text { Pulegone }\end{array}$ & $1^{\mathrm{a}}$ & 30 & [131] & $\star \star$ & - & - & - \\
\hline
\end{tabular}


Table 1. Cont.

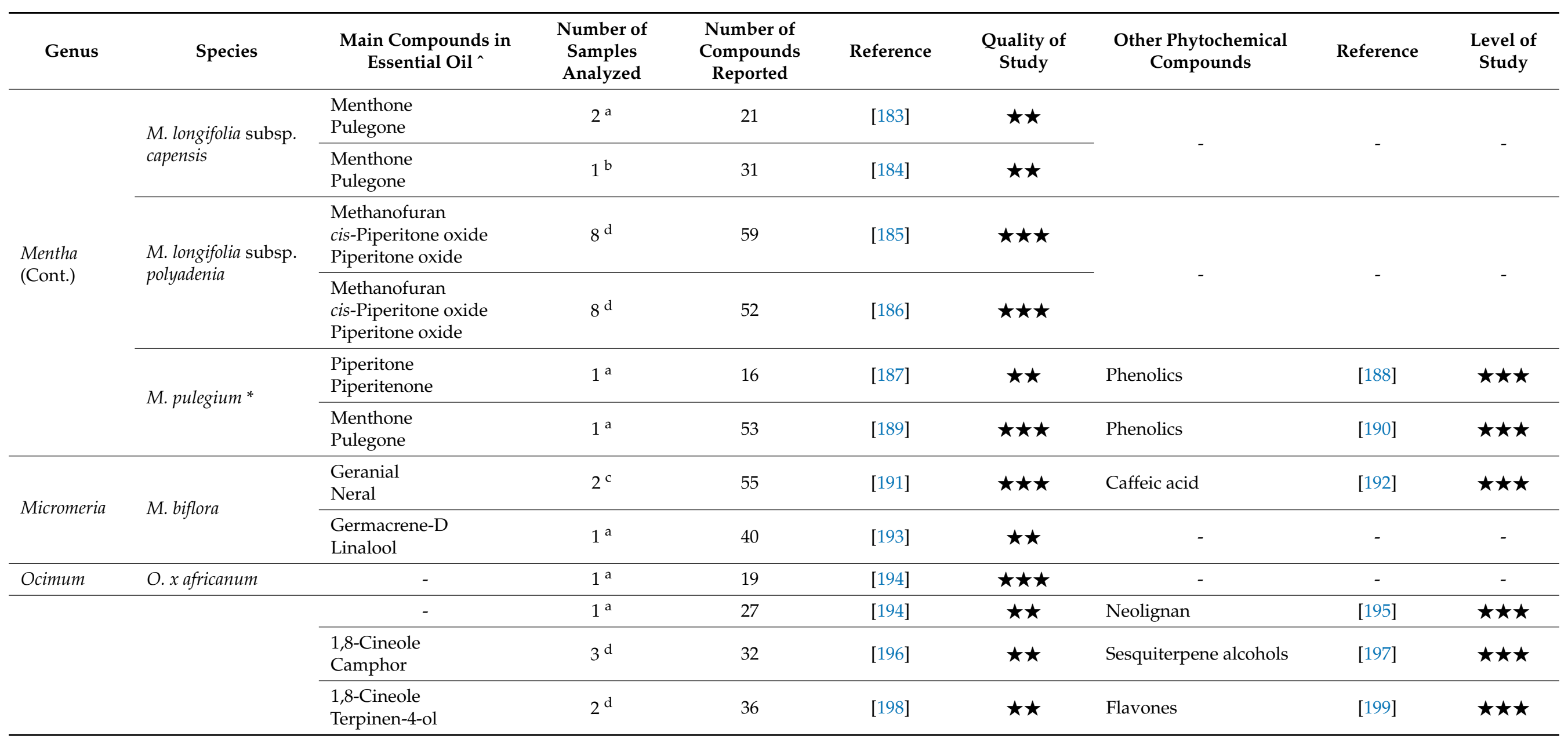


Table 1. Cont.

\begin{tabular}{|c|c|c|c|c|c|c|c|c|c|}
\hline Genus & Species & $\begin{array}{l}\text { Main Compounds in } \\
\text { Essential Oil ^ }\end{array}$ & $\begin{array}{l}\text { Number of } \\
\text { Samples } \\
\text { Analyzed }\end{array}$ & $\begin{array}{l}\text { Number of } \\
\text { Compounds } \\
\text { Reported }\end{array}$ & Reference & $\begin{array}{l}\text { Quality of } \\
\text { Study }\end{array}$ & $\begin{array}{l}\text { Other Phytochemical } \\
\text { Compounds }\end{array}$ & Reference & $\begin{array}{l}\text { Level of } \\
\text { Study }\end{array}$ \\
\hline \multirow{8}{*}{$\begin{array}{l}\text { Ocimum } \\
\text { (Cont.) }\end{array}$} & \multirow{5}{*}{ O. americanum } & $\begin{array}{l}\text { Eugenol } \\
\text { Methyl carvacrol } \\
\text { Terpineol }\end{array}$ & $4^{b, c}$ & 17 & [200] & $\star \star$ & \multirow{5}{*}{ - } & \multirow{5}{*}{-} & \multirow{5}{*}{-} \\
\hline & & $\begin{array}{l}\text { cis- } \beta \text {-Ocimene } \\
\text { Estragol } \\
\beta \text {-Bisabolene }\end{array}$ & $1^{\mathrm{a}}$ & 22 & [201] & $\star \star$ & & & \\
\hline & & Camphor & $1^{\mathrm{c}}$ & 51 & [202] & $\star \star \star$ & & & \\
\hline & & $\begin{array}{l}\text { Carvone } \\
\text { Elemol } \\
\alpha \text {-Humulene }\end{array}$ & $1^{\mathrm{a}}$ & 41 & [131] & $\star \star$ & & & \\
\hline & & $\begin{array}{l}\text { 1,8-Cineole } \\
\text { (Z)-Methyl cinnamate }\end{array}$ & $1^{\mathrm{a}}$ & 14 & [203] & $\star \star$ & & & \\
\hline & O. gratissimum & $\begin{array}{l}\text { Eugenol } \\
\text { Germacrene-D }\end{array}$ & $1^{c}$ & 35 & [202] & $\star \star$ & Caffeic acid esters & [42] & $\star$ \\
\hline & O. labiatum & - & - & - & - & - & Terpenoids & [205] & $\star \star \star \star$ \\
\hline & O. obovatum & - & $1^{a}$ & 43 & [206] & $\star \star$ & Terpenoid saponins & [207] & $\star \star \star$ \\
\hline Orthosiphon & O. thymiflorus & $\begin{array}{l}\text { 2-isopropyl-5-methyl-9- } \\
\text { methylene-bicyclo-1- } \\
\text { decene(4.4.0) }\end{array}$ & $1^{\mathrm{a}}$ & 33 & [208] & $\star \star$ & - & - & - \\
\hline \multirow[t]{2}{*}{ Platostoma } & \multirow[t]{2}{*}{ P. rotundifolium } & $\begin{array}{l}\text { Germacrene-D } \\
\beta \text {-Caryophyllene } \\
\beta \text {-Gurjunene }\end{array}$ & $1^{\mathrm{d}}$ & 24 & [209] & $\star \star$ & \multirow[t]{2}{*}{ Terpenoids } & \multirow[t]{2}{*}{ [210] } & \multirow[t]{2}{*}{$\#$} \\
\hline & & Spathulenol & $1^{\mathrm{a}}$ & 59 & [211] & $\star \star \star \star$ & & & \\
\hline
\end{tabular}


Table 1. Cont.

\begin{tabular}{|c|c|c|c|c|c|c|c|c|c|}
\hline Genus & Species & $\begin{array}{l}\text { Main Compounds in } \\
\text { Essential Oil }\end{array}$ & $\begin{array}{c}\text { Number of } \\
\text { Samples } \\
\text { Analyzed }\end{array}$ & $\begin{array}{l}\text { Number of } \\
\text { Compounds } \\
\text { Reported }\end{array}$ & Reference & $\begin{array}{l}\text { Quality of } \\
\text { Study }\end{array}$ & $\begin{array}{c}\text { Other Phytochemical } \\
\text { Compounds }\end{array}$ & Reference & $\begin{array}{c}\text { Level of } \\
\text { Study }\end{array}$ \\
\hline \multirow{15}{*}{ Plectranthus } & \multirow{4}{*}{ P. ambiguus } & \multirow{4}{*}{-} & & \multirow{4}{*}{-} & \multirow{4}{*}{-} & \multirow{4}{*}{ - } & Terpenoids & [58] & $\#$ \\
\hline & & & & & & & Caffeic acid esters & [42] & $\star$ \\
\hline & & & & & & & Flavonoids & [90] & $\star \star \star \star$ \\
\hline & & & & & & & Phyllocladanes & [212] & $\star \star \star \star$ \\
\hline & P. cilliatus & $\begin{array}{l}\text { Spathulenol } \\
\text { Bicyclogermacrene } \\
\delta \text {-Cadinine }\end{array}$ & $2^{d}$ & 106 & [213] & $\star \star \star \star$ & Terpenoids & {$[58]$} & $\#$ \\
\hline & \multirow{2}{*}{ P. ecklonii } & \multirow{2}{*}{-} & \multirow{2}{*}{-} & \multirow{2}{*}{-} & \multirow{2}{*}{-} & \multirow{2}{*}{-} & Terpenoids & [58] & $\#$ \\
\hline & & & & & & & Flavonoids & [90] & $\star \star \star$ \\
\hline & P. ernestii & - & - & - & - & - & Terpenoids & [212] & $\#$ \\
\hline & \multirow{2}{*}{ P. fruticosus } & \multirow{2}{*}{ Sabinyl acetate } & \multirow{2}{*}{$1^{c}$} & \multirow{2}{*}{52} & \multirow{2}{*}{ [215] } & \multirow{2}{*}{$\star \star \star$} & Terpenoids & [216] & $\star \star \star \star$ \\
\hline & & & & & & & Terpenoids & [210] & $\#$ \\
\hline & \multirow{2}{*}{ P. lucidus } & \multirow{2}{*}{-} & \multirow{2}{*}{-} & \multirow{2}{*}{-} & \multirow{2}{*}{-} & \multirow{2}{*}{-} & Terpenoids & [96] & $\star \star \star \star$ \\
\hline & & & & & & & Terpenoids & [97] & $\star \star \star$ \\
\hline & P. praetermissus & - & - & - & - & - & Terpenoids & [96] & $\star \star \star \star$ \\
\hline & P. purpuratus & - & - & - & - & - & $\begin{array}{l}\text { Diterpenoid } \\
\text { quinomethans } \\
\text { Vinylogous quinones } \\
\text { Phyllocladene } \\
\text { derivative }\end{array}$ & [217] & $\star \star \star$ \\
\hline & $\begin{array}{l}\text { P. purpuratus subsp. } \\
\text { purpuratus }\end{array}$ & - & - & - & - & - & Terpenoids & [97] & $\star \star$ \\
\hline
\end{tabular}


Table 1. Cont.

\begin{tabular}{|c|c|c|c|c|c|c|c|c|c|}
\hline Genus & Species & $\begin{array}{l}\text { Main Compounds in } \\
\text { Essential Oil ^ }\end{array}$ & $\begin{array}{c}\text { Number of } \\
\text { Samples } \\
\text { Analyzed }\end{array}$ & $\begin{array}{l}\text { Number of } \\
\text { Compounds } \\
\text { Reported }\end{array}$ & Reference & $\begin{array}{l}\text { Quality of } \\
\text { Study }\end{array}$ & $\begin{array}{l}\text { Other Phytochemical } \\
\text { Compounds }\end{array}$ & Reference & $\begin{array}{l}\text { Level of } \\
\text { Study }\end{array}$ \\
\hline \multirow{11}{*}{$\begin{array}{l}\text { Plectranthus } \\
\text { (Cont.) }\end{array}$} & \multirow{2}{*}{ P. saccatus } & \multirow{2}{*}{-} & \multirow{2}{*}{-} & \multirow{2}{*}{-} & \multirow{2}{*}{-} & \multirow{2}{*}{-} & Terpenoids & [218] & $\star \star$ \\
\hline & & & & & & & Terpenoids & [219] & 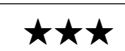 \\
\hline & \multirow{3}{*}{ P. strigosus } & \multirow{3}{*}{-} & \multirow{3}{*}{-} & \multirow{3}{*}{-} & \multirow{3}{*}{-} & \multirow{3}{*}{-} & Terpenoids & [58] & $\#$ \\
\hline & & & & & & & Terpenoids & [210] & $\#$ \\
\hline & & & & & & & Terpenoids & [220] & $\#$ \\
\hline & \multirow{2}{*}{ P. verticillatus } & \multirow{2}{*}{-} & \multirow{2}{*}{-} & \multirow{2}{*}{-} & \multirow{2}{*}{-} & \multirow{2}{*}{-} & Terpenoids & [221] & $\star \star$ \\
\hline & & & & & & & Terpenoids & [222] & $\star \star \star$ \\
\hline & \multirow{4}{*}{ P. zuluensis } & \multirow{4}{*}{-} & \multirow{4}{*}{-} & \multirow{4}{*}{-} & \multirow{4}{*}{-} & \multirow{4}{*}{-} & Terpenoids & [221] & $\star \star$ \\
\hline & & & & & & & Terpenoids & [96] & $\star \star \star$ \\
\hline & & & & & & & Terpenoids & [213] & $\star \star \star \star$ \\
\hline & & & & & & & Terpenoids & [219] & $\star \star \star \star$ \\
\hline \multirow{4}{*}{ Prunella* } & \multirow{4}{*}{ P. vulgaris * } & Aromadendrene & $1^{\mathrm{c}}$ & 28 & [225] & $\star \star$ & Terpenoids & [226] & $\star \star \star \star ~$ \\
\hline & & \multirow{2}{*}{-} & \multirow{2}{*}{-} & \multirow{2}{*}{-} & \multirow{2}{*}{-} & \multirow{2}{*}{-} & Terpenoids & [227] & $\star \star \star$ \\
\hline & & & & & & & Terpenoids & [228] & $\star \star \star \star$ \\
\hline & & - & - & - & - & - & $\begin{array}{l}\text { Triterpenoids } \\
\text { Saponins } \\
\text { Sterols } \\
\text { Flavonoids } \\
\text { Coumarins } \\
\text { Phenylpropanoids }\end{array}$ & [229] & $\#$ \\
\hline
\end{tabular}


Table 1. Cont.

\begin{tabular}{|c|c|c|c|c|c|c|c|c|c|}
\hline Genus & Species & $\begin{array}{l}\text { Main Compounds in } \\
\text { Essential Oil }\end{array}$ & $\begin{array}{l}\text { Number of } \\
\text { Samples } \\
\text { Analyzed }\end{array}$ & $\begin{array}{l}\text { Number of } \\
\text { Compounds } \\
\text { Reported }\end{array}$ & Reference & $\begin{array}{l}\text { Quality of } \\
\text { Study }\end{array}$ & $\begin{array}{l}\text { Other Phytochemical } \\
\text { Compounds }\end{array}$ & Reference & $\begin{array}{l}\text { Level of } \\
\text { Study }\end{array}$ \\
\hline \multirow{3}{*}{ Pseudodictamnus } & \multirow{3}{*}{ P. africanus } & \multirow{3}{*}{-} & \multirow{3}{*}{-} & \multirow{3}{*}{-} & \multirow{3}{*}{-} & \multirow{3}{*}{-} & Terpenes & [230] & $\star \star \star$ \\
\hline & & & & & & & $\begin{array}{l}\text { Terpenes } \\
\text { Phenolics }\end{array}$ & [231] & $\star \star \star$ \\
\hline & & & & & & & Terpenes & [232] & $\star \star \star \star$ \\
\hline \multirow{3}{*}{ Rotheca } & R. myricoides & - & - & - & - & - & Cyclohexapeptide & [233] & $\star \star \star \star$ \\
\hline & \multirow{2}{*}{ R. wildii } & \multirow{2}{*}{-} & \multirow{2}{*}{-} & \multirow{2}{*}{-} & \multirow{2}{*}{-} & \multirow{2}{*}{-} & Saponins & [234] & $\#$ \\
\hline & & & & & & & Triterpenoid Saponins & [235] & $\#$ \\
\hline \multirow{8}{*}{ Salvia } & \multirow{4}{*}{ S. africana } & $\begin{array}{l}p \text {-Cymene } \\
\alpha \text {-Eudesmol } \\
\gamma \text {-Terpinene }\end{array}$ & $1^{b}$ & 53 & [236] & $\star \star \star$ & Diterpenoids & [237] & $\star \star$ \\
\hline & & $\begin{array}{l}\text { Caryophyllene oxide } \\
\beta \text {-Caryophyllene }\end{array}$ & $1^{\mathrm{a}}$ & 56 & [238] & $\star \star \star$ & Terpenoids & [204] & $\star \star$ \\
\hline & & $\begin{array}{l}\text { Caryophyllene oxide } \\
\text { Spathulenol }\end{array}$ & $1^{\mathrm{a}}$ & 45 & [239] & $\star \star$ & \multirow{2}{*}{ Phenolics } & \multirow{2}{*}{ [240] } & \multirow{2}{*}{$\star \star \star \star$} \\
\hline & & $\begin{array}{l}\text { Limonene } \\
\text { Viridiflorol }\end{array}$ & $12^{d}$ & 46 & [241] & $\star \star$ & & & \\
\hline & S. albicaulis & Viridiflorol & $1^{\mathrm{a}}$ & 38 & [242] & $\star \star$ & Phenolics & [240] & $\star \star \star \star$ \\
\hline & \multirow{3}{*}{ S. aurea } & $\begin{array}{l}\text { Caryophyllene oxide } \\
\alpha \text {-Eudesmol } \\
\beta \text {-Eudesmol }\end{array}$ & $1^{\mathrm{a}}$ & 48 & [238] & $\star \star$ & Terpenoids & [243] & $\star \star$ \\
\hline & & Myrcene & $1^{a}$ & 43 & [239] & $\star \star$ & Phenolics & [244] & $\star \star$ \\
\hline & & $\begin{array}{l}\text { Terpinene-4-ol + } \\
\beta \text {-Caryophyllene } \\
\alpha \text {-Eudesmol } \\
\beta \text {-Eudesmol }\end{array}$ & $6^{\mathrm{d}}$ & 20 & [245] & $\star \star$ & Phenolics & [240] & $\star \star \star$ \\
\hline
\end{tabular}


Table 1. Cont.

\begin{tabular}{|c|c|c|c|c|c|c|c|c|c|}
\hline Genus & Species & $\begin{array}{l}\text { Main Compounds in } \\
\text { Essential Oil ^ }\end{array}$ & $\begin{array}{l}\text { Number of } \\
\text { Samples } \\
\text { Analyzed }\end{array}$ & $\begin{array}{l}\text { Number of } \\
\text { Compounds } \\
\text { Reported }\end{array}$ & Reference & $\begin{array}{l}\text { Quality of } \\
\text { Study }\end{array}$ & $\begin{array}{l}\text { Other Phytochemical } \\
\text { Compounds }\end{array}$ & Reference & $\begin{array}{l}\text { Level of } \\
\text { Study }\end{array}$ \\
\hline & & $\begin{array}{l}\text { Limonene } \\
\alpha \text {-Humulene } \\
\beta \text {-Caryophyllene }+ \\
\text { Terpinen-4-ol } \\
\tau \text {-Cadinol }\end{array}$ & $1^{\mathrm{a}}$ & 43 & [131] & $\star \star$ & - & - & - \\
\hline & S. aurita & - & - & - & - & - & Phenolics & [240] & 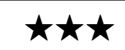 \\
\hline \multirow{9}{*}{$\begin{array}{l}\text { Salvia } \\
\text { (Cont.) }\end{array}$} & \multirow{3}{*}{ S. chamelaeagnea } & $\begin{array}{l}\text { 1,8-Cineole } \\
\alpha \text {-Pinene }\end{array}$ & $1^{\mathrm{a}}$ & 43 & [239] & $\star \star$ & Terpenoids & [210] & $\#$ \\
\hline & & \multirow{2}{*}{ Viridiflorol } & \multirow{2}{*}{$5^{d}$} & \multirow{2}{*}{18} & \multirow{2}{*}{ [245] } & \multirow{2}{*}{$\star \star$} & Phenolics & [240] & $\star \star \star$ \\
\hline & & & & & & & Terpenoids & [246] & $\star \star \star \star$ \\
\hline & S. coccinea & $\begin{array}{l}\text { 2,5-Dimethoxy- } p \text { - } \\
\text { cymene } \\
\text { Acenaphthene } \\
\text { Aromadendrene } \\
\text { Globulol }\end{array}$ & $3^{c}$ & 21 & [247] & $\star \star \star$ & Phenolics & [248] & $\star \star$ \\
\hline & S. dentata & - & - & - & - & - & Phenolic diterpene & [249] & $\star$ \\
\hline & \multirow{2}{*}{ S. disermas } & $\begin{array}{l}\text { Linalyl acetate } \\
\text { Shyobunone }\end{array}$ & $3^{d}$ & 28 & [238] & $\star \star$ & Phenolic diterpene & [249] & $\star$ \\
\hline & & $\begin{array}{l}\text { Linalool } \\
\text { Linalyl acetate }\end{array}$ & $1^{\mathrm{a}}$ & 42 & [131] & $\star \star$ & Phenolics & [240] & $\star \star \star \star$ \\
\hline & \multirow{2}{*}{ S. dolomitica } & $\begin{array}{l}\text { 1,8-Cineole } \\
\text { Borneol } \\
\beta \text {-Caryophyllene }\end{array}$ & $3^{b, d}$ & 110 & [250] & $\star \star \star$ & \multirow{2}{*}{ Phenolics } & \multirow{2}{*}[240]{} & \multirow{2}{*}{$\star \star \star$} \\
\hline & & $\begin{array}{l}\text {-Caryophyllene } \\
\text { Limonene } \\
\text { Germacrene-D }\end{array}$ & $3^{b}$ & 37 & [251] & $\star \star$ & & & \\
\hline
\end{tabular}


Table 1. Cont.

\begin{tabular}{|c|c|c|c|c|c|c|c|c|c|}
\hline Genus & Species & $\begin{array}{l}\text { Main Compounds in } \\
\text { Essential Oil }\end{array}$ & $\begin{array}{c}\text { Number of } \\
\text { Samples } \\
\text { Analyzed }\end{array}$ & $\begin{array}{l}\text { Number of } \\
\text { Compounds } \\
\text { Reported }\end{array}$ & Reference & $\begin{array}{l}\text { Quality of } \\
\text { Study }\end{array}$ & $\begin{array}{l}\text { Other Phytochemical } \\
\text { Compounds }\end{array}$ & Reference & $\begin{array}{c}\text { Level of } \\
\text { Study }\end{array}$ \\
\hline & & $\begin{array}{l}\text { 1,8-Cineole } \\
\beta \text {-Caryophyllene }\end{array}$ & $1^{\mathrm{a}, \mathrm{b}}$ & 46 & [252] & $\star \star$ & & & \\
\hline & & $\begin{array}{l}\text { 1,8-Cineole } \\
\beta \text {-Caryophyllene }\end{array}$ & $12^{d}$ & 66 & [238] & $\star \star \star$ & & & \\
\hline & & Linalool & $1^{\mathrm{a}}$ & 34 & [242] & $\star \star$ & - & - & - \\
\hline & S. lanceolata & $\begin{array}{l}\text { Sabinene } \\
\text { Spathulenol }\end{array}$ & $1^{a}$ & 41 & {$[238]$} & $\star \star$ & Phenolic diterpene & [249] & $\star$ \\
\hline \multirow{8}{*}{$\begin{array}{l}\text { Salvia } \\
\text { (Cont.) }\end{array}$} & \multirow{3}{*}{ S. lanceolata (Cont.) } & $\begin{array}{l}\text { Caryophyllene oxide } \\
\text { Spathulenol }\end{array}$ & $1^{\mathrm{a}}$ & 43 & [239] & $\star \star$ & Phenolics & [240] & $\star \star \star \star$ \\
\hline & & $\beta$-Caryophyllene & $12^{d}$ & 48 & [241] & $\star \star$ & \multirow[b]{2}{*}{-} & \multirow[b]{2}{*}{-} & \multirow[b]{2}{*}{-} \\
\hline & & $\begin{array}{l}\text { Bicyclogermacrene } \\
\text { Terpinene-4-ol + } \\
\beta \text {-Caryophyllene }\end{array}$ & $5^{d}$ & 15 & [245] & $\star \star$ & & & \\
\hline & S. muirii & $\begin{array}{l}\text { 1,8-Cineole } \\
\text { Limonene } \\
\alpha \text {-Pinene }\end{array}$ & $1^{\mathrm{a}}$ & 39 & [253] & $\star \star$ & Phenolics & [240] & $\star \star \star$ \\
\hline & \multirow{3}{*}{ S. namaensis } & $\begin{array}{l}\text { Camphene } \\
\text { Camphor }\end{array}$ & $1^{a}$ & 55 & [238] & $\star \star \star \star$ & Phenolic diterpene & [249] & $\star$ \\
\hline & & $\begin{array}{l}\text { 1,8-Cineole } \\
\text { Camphene } \\
\text { Camphor } \\
\alpha \text {-Pinene }\end{array}$ & $1^{a}$ & 20 & [254] & $\star \star$ & \multirow[t]{2}{*}{ Phenolics } & \multirow[t]{2}{*}{ [240] } & \multirow[t]{2}{*}{$\star \star \star \star$} \\
\hline & & Camphor & $1^{a}$ & 64 & [131] & $\star \star \star$ & & & \\
\hline & S. radula & - & - & - & - & - & Phenolics & [240] & $\star \star \star \star$ \\
\hline
\end{tabular}


Table 1. Cont.

\begin{tabular}{|c|c|c|c|c|c|c|c|c|c|}
\hline Genus & Species & $\begin{array}{l}\text { Main Compounds in } \\
\text { Essential Oil ^ }\end{array}$ & $\begin{array}{l}\text { Number of } \\
\text { Samples } \\
\text { Analyzed }\end{array}$ & $\begin{array}{l}\text { Number of } \\
\text { Compounds } \\
\text { Reported }\end{array}$ & Reference & $\begin{array}{l}\text { Quality of } \\
\text { Study }\end{array}$ & $\begin{array}{l}\text { Other Phytochemical } \\
\text { Compounds }\end{array}$ & Reference & $\begin{array}{l}\text { Level of } \\
\text { Study }\end{array}$ \\
\hline & \multirow{4}{*}{ S. repens } & $\begin{array}{l}\text { Ledol } \\
\alpha \text {-Bisabolol } \\
\beta \text {-Phellandrene } \\
\delta \text {-3-Carene } \\
\text { E-Nerolidol }\end{array}$ & $6^{\mathrm{d}}$ & 106 & [255] & $\star \star \star$ & Phenolic diterpene & [249] & $\star$ \\
\hline & & $\begin{array}{l}\beta \text {-Phellandrene } \\
\beta \text {-Caryophyllene }\end{array}$ & $1^{\mathrm{a}}$ & 55 & [256] & $\star \star \star$ & Terpenoids & [257] & $\star \star \star \star$ \\
\hline & & $\begin{array}{l}\text { Ledol } \\
\alpha \text {-Bisabolol } \\
\beta \text {-Caryophyllene } \\
\beta \text {-Phellandrene }\end{array}$ & $4^{\mathrm{d}}$ & 90 & [258] & $\star \star \star$ & Terpenoids & [259] & $\star \star \star$ \\
\hline & & E-Nerolidol & & & & & Phenolics & [240] & $\star \star \star$ \\
\hline \multirow{6}{*}{$\begin{array}{l}\text { Salvia } \\
\text { (Cont.) }\end{array}$} & \multirow{6}{*}{ S. runcinata } & $\begin{array}{l}\text { Caryophyllene } \\
\text { Ledol } \\
\beta \text {-Bisabolone }\end{array}$ & $2^{b}$ & 26 & [260] & $\star \star$ & Phenolic diterpene & [249] & $\star$ \\
\hline & & $\begin{array}{l}\alpha \text {-Pinene } \\
\beta \text {-Caryophyllene } \\
\text { E-Nerolidol }\end{array}$ & $15^{\mathrm{d}}$ & 157 & [255] & $\star \star \star$ & \multirow{5}{*}{ Phenolics } & \multirow{5}{*}{ [240] } & \multirow{5}{*}{$\star \star \star \star$} \\
\hline & & $\begin{array}{l}\alpha \text {-Bisabolol } \\
\beta \text {-Caryophyllene }\end{array}$ & $1^{\mathrm{a}}$ & 73 & [256] & $\star \star \star \star$ & & & \\
\hline & & $\begin{array}{l}\text { Limonene } \\
\text { Nerolidol } \\
\alpha \text {-Bisabolol } \\
\beta \text {-Caryophyllene } \\
\beta \text {-Eudesmol } \\
\delta \text {-3-Carene }\end{array}$ & $20^{d}$ & $11(x>5 \%)$ & [261] & $\star \star$ & & & \\
\hline & & $\begin{array}{l}\text { Viridiflorol } \\
\beta \text {-Caryophyllene }\end{array}$ & $1^{\mathrm{a}}$ & 44 & [131] & $\star \star$ & & & \\
\hline & & $\begin{array}{l}\alpha \text {-Bisabolol } \\
\beta \text {-Caryophyllene } \\
\text { E-Nerolidol }\end{array}$ & $12^{\mathrm{d}}$ & 118 & [258] & $\star \star \star \star$ & & & \\
\hline
\end{tabular}


Table 1. Cont.

\begin{tabular}{|c|c|c|c|c|c|c|c|c|c|}
\hline Genus & Species & $\begin{array}{l}\text { Main Compounds in } \\
\text { Essential Oil }\end{array}$ & $\begin{array}{l}\text { Number of } \\
\text { Samples } \\
\text { Analyzed }\end{array}$ & $\begin{array}{c}\text { Number of } \\
\text { Compounds } \\
\text { Reported }\end{array}$ & Reference & $\begin{array}{l}\text { Quality of } \\
\text { Study }\end{array}$ & $\begin{array}{l}\text { Other Phytochemical } \\
\text { Compounds }\end{array}$ & Reference & $\begin{array}{l}\text { Level of } \\
\text { Study }\end{array}$ \\
\hline & S. scabra & - & - & - & - & - & Phenolic diterpene & [249] & $\star$ \\
\hline & S. schlechteri & - & - & - & - & - & Phenolics & [240] & $\star \star \star$ \\
\hline & \multirow{4}{*}{ S. stenophylla } & $\begin{array}{l}\text { Myrcene } \\
\alpha \text {-Bisabolol }\end{array}$ & $1^{\mathrm{a}}$ & 34 & [238] & $\star \star$ & Phenolic diterpene & [249] & $\star$ \\
\hline & & $\begin{array}{l}\alpha \text {-Bisabolol } \\
\delta \text {-3-Carene }\end{array}$ & $10^{\mathrm{d}}$ & 128 & [255] & $\star \star \star \star$ & Flavonoids & [262] & $\star \star$ \\
\hline & & $\begin{array}{l}(+)-\alpha \text {-Bisabolol } \\
\alpha \text {-Phellandrene }\end{array}$ & $1^{\mathrm{a}}$ & 31 & [263] & $\star \star$ & \multirow[t]{2}{*}{ Phenolics } & \multirow[t]{2}{*}{ [240] } & \multirow[t]{2}{*}{$\star \star \star$} \\
\hline & & $\begin{array}{l}\delta \text {-3-Carene } \\
\text { Manool }\end{array}$ & $1^{\mathrm{a}}$ & 59 & [256] & $\star \star \star$ & & & \\
\hline \multirow{5}{*}{$\begin{array}{l}\text { Salvia } \\
\text { (Cont.) }\end{array}$} & \multirow[t]{2}{*}{ S. stenophylla (Cont.) } & $\begin{array}{l}\delta \text {-3-Carene } \\
\text { Mycrene } \\
\text { Limonene } \\
\beta \text {-Phellandrene } \\
\gamma \text {-Terpinene } \\
p \text {-Cymene } \\
\text { Nerolidiol } \\
\alpha \text {-Bisabolol } \\
\end{array}$ & $27^{\mathrm{d}}$ & $12(x>5 \%)$ & {$[261]$} & $\star \star$ & \multirow[t]{2}{*}{-} & \multirow[t]{2}{*}{-} & \multirow[t]{2}{*}{-} \\
\hline & & $\begin{array}{l}\delta \text {-3-Carene } \\
p \text {-Cymene } \\
\text { (E)-nerolidiol } \\
\alpha \text {-Bisabolol }\end{array}$ & $12^{d}$ & 128 & [258] & $\star \star \star \star$ & & & \\
\hline & \multirow{3}{*}{ S. verbenaca* } & - & $1^{\mathrm{a}}$ & 76 & [264] & $\star \star \star \star$ & Terpenoids & [210] & $\#$ \\
\hline & & - & $2^{a, b}$ & 63 & [265] & $\star \star \star$ & Phenolics & [266] & $\star \star$ \\
\hline & & - & $1^{\mathrm{a}}$ & 18 & [267] & $\star \star$ & - & - & - \\
\hline Satureja * & S. thymbra* & $\begin{array}{l}p \text {-Cymene } \\
\gamma \text {-Terpinene } \\
\text { Thymol } \\
\text { Carvacrol }\end{array}$ & $7^{c}$ & 40 & [268] & $\star \star$ & Phenolics & [269] & $\star \star$ \\
\hline
\end{tabular}


Table 1. Cont.

\begin{tabular}{|c|c|c|c|c|c|c|c|c|c|}
\hline Genus & Species & $\begin{array}{l}\text { Main Compounds in } \\
\text { Essential Oil }\end{array}$ & $\begin{array}{l}\text { Number of } \\
\text { Samples } \\
\text { Analyzed }\end{array}$ & $\begin{array}{l}\text { Number of } \\
\text { Compounds } \\
\text { Reported }\end{array}$ & Reference & $\begin{array}{l}\text { Quality of } \\
\text { Study }\end{array}$ & $\begin{array}{c}\text { Other Phytochemical } \\
\text { Compounds }\end{array}$ & Reference & $\begin{array}{l}\text { Level of } \\
\text { Study }\end{array}$ \\
\hline Stachys & S. aethiopica & - & - & - & - & - & $\begin{array}{l}\text { Saponins } \\
\text { Tannins }\end{array}$ & [270] & $\star \star$ \\
\hline \multirow[b]{2}{*}{ Syncolostemon } & S. argenteus & - & - & - & - & - & Pyrones & [271] & $\star \star \star \star$ \\
\hline & S. bracteosus & - & - & - & - & - & $\begin{array}{l}\text { Alcohols } \\
\text { Acids } \\
\text { Esters } \\
\text { Lactones } \\
\text { Phenolics } \\
\text { Sesquiterpenes }\end{array}$ & [272] & $\star \star$ \\
\hline \multirow{7}{*}{$\begin{array}{l}\text { Syncolostemon } \\
\text { (Cont.) }\end{array}$} & \multirow{2}{*}{ S. densiflorus } & \multirow[t]{2}{*}{ - } & \multirow{2}{*}{-} & \multirow[t]{2}{*}{-} & \multirow{2}{*}{-} & \multirow{2}{*}{-} & Pyrones & [37] & $\star \star \star$ \\
\hline & & & & & & & Pyrones & [273] & $\star \star \star \star$ \\
\hline & S. modestus & $\begin{array}{l}p \text {-Cymene } \\
\text { Spathulenol } \\
\text { Viridiflorol } \\
\gamma \text {-Terpinene }\end{array}$ & $1^{\mathrm{a}}$ & 10 & [131] & $\star \star$ & - & - & - \\
\hline & S. parviflorus & - & - & - & - & - & Pyrones & [231] & 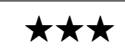 \\
\hline & S. petiolatus & $\begin{array}{l}\text { (+)-Linalool } \\
\text { (E)- } \beta \text {-farnesene } \\
(+) \text {-Bicyclogermacrene } \\
(-) \text {-Germacrene D }\end{array}$ & $1^{\mathrm{a}}$ & $\mathrm{N} / \mathrm{A}$ & [274] & $\star \star$ & - & - & - \\
\hline & S. pretoriae & $\beta$-Caryophyllene & $1^{a}$ & 6 & [131] & $\star \star \star$ & - & - & - \\
\hline & S. rotundifolius & - & - & - & - & - & Pyrones & [231] & $\star \star \star \star$ \\
\hline \multirow{3}{*}{ Tetradenia } & T. barberae & - & - & - & - & - & Pyrones & [275] & $\star \star \star \star$ \\
\hline & \multirow[t]{2}{*}{ T. riparia } & $\begin{array}{l}\text { 14-Hydroxy-9-epi-(E)- } \\
\text { Caryophyllene } \\
\text { 6,7-Dehydroroyleanone } \\
\alpha \text {-Cadinol }\end{array}$ & $2^{c}$ & 51 & {$[276]$} & $\star \star \star$ & Pyrones & [35] & $\star \star \star$ \\
\hline & & $\begin{array}{l}\text { Fenchone } \\
\delta \text {-Cadinene }\end{array}$ & $1^{\mathrm{a}}$ & 49 & [277] & $\star \star$ & Pyrones & [278] & $\star \star \star$ \\
\hline
\end{tabular}


Table 1. Cont

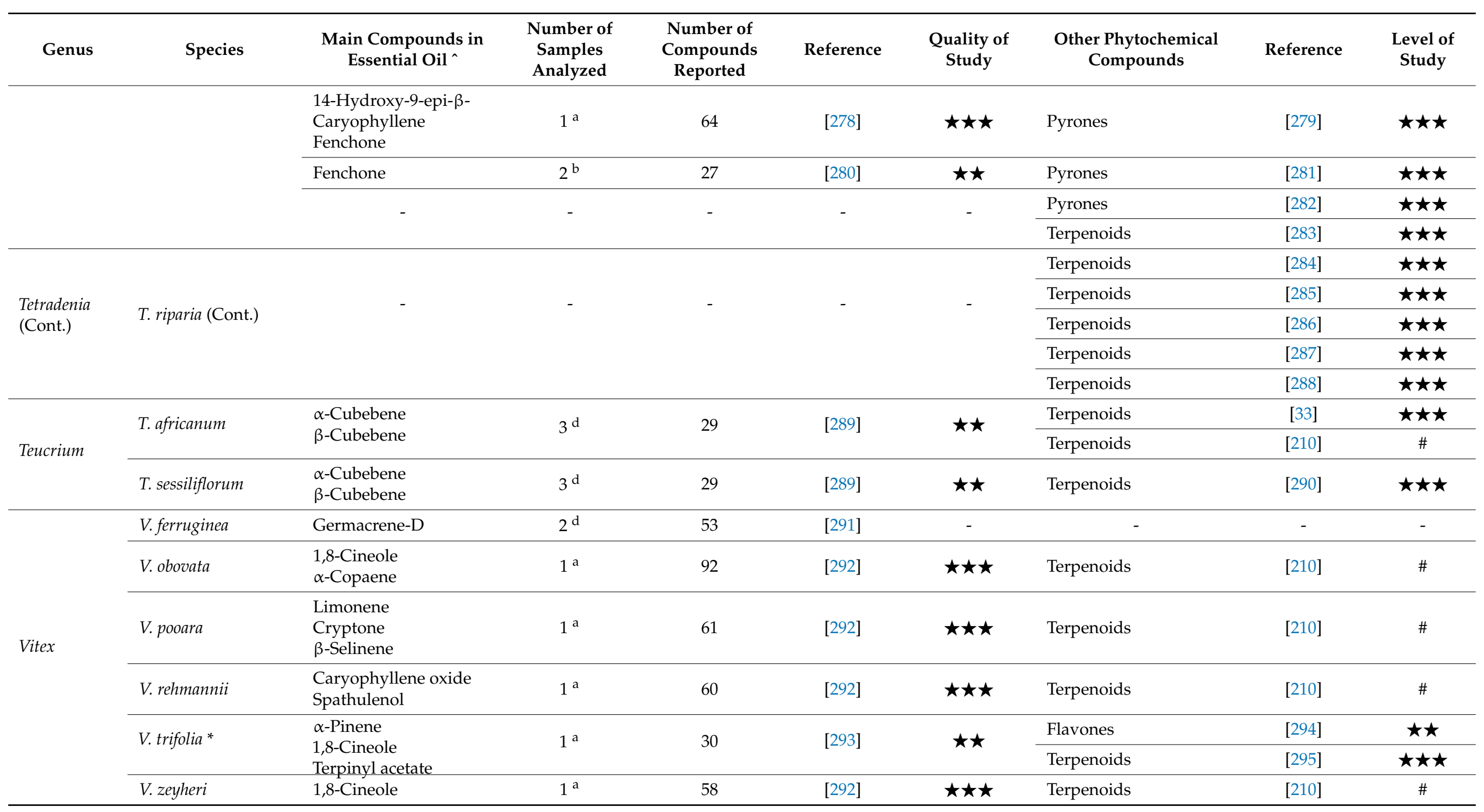


Table 1. Cont.

\begin{tabular}{|c|c|c|c|c|c|c|c|c|c|}
\hline Genus & Species & $\begin{array}{l}\text { Main Compounds in } \\
\text { Essential Oil ^ }\end{array}$ & $\begin{array}{l}\text { Number of } \\
\text { Samples } \\
\text { Analyzed }\end{array}$ & $\begin{array}{l}\text { Number of } \\
\text { Compounds } \\
\text { Reported }\end{array}$ & Reference & $\begin{array}{l}\text { Quality of } \\
\text { Study }\end{array}$ & $\begin{array}{l}\text { Other Phytochemical } \\
\text { Compounds }\end{array}$ & Reference & $\begin{array}{l}\text { Level of } \\
\text { Study }\end{array}$ \\
\hline \multirow{4}{*}{ Volkameria } & \multirow{4}{*}{ V. glabra } & \multirow{4}{*}{-} & \multirow{4}{*}{-} & \multirow{4}{*}{-} & \multirow{4}{*}{-} & \multirow{4}{*}{-} & Terpenoids & [210] & $\#$ \\
\hline & & & & & & & Terpenoids & [296] & 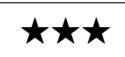 \\
\hline & & & & & & & Terpenoids & [297] & $\star \star$ \\
\hline & & & & & & & Terpenoids & [298] & $\star \star \star$ \\
\hline
\end{tabular}

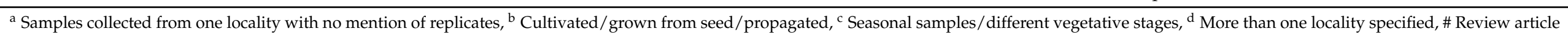

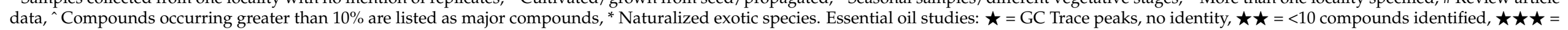

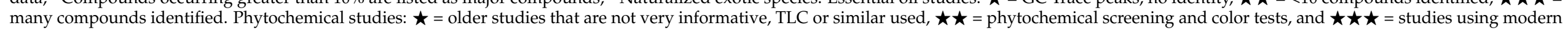

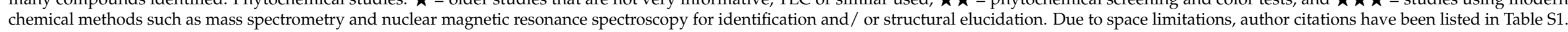


Alkaloids

Quinones
Coumarins Saponins
Flavonoids/ Phenolics Tannins
Pyrones

Terpenoids \& Sterols

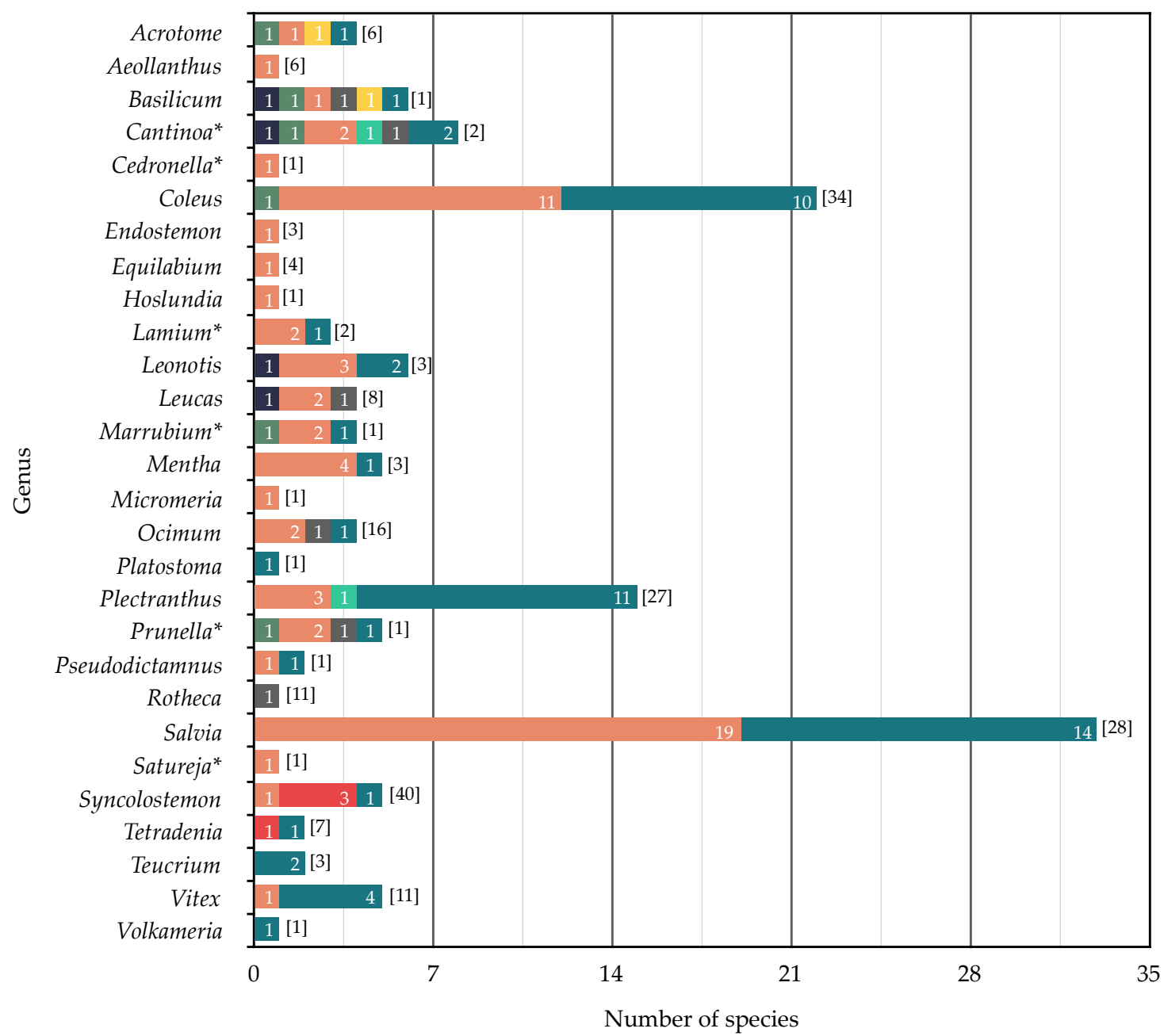

Figure 5. The numbers of species from 28 genera (out of 42) of southern African Lamiaceae that have been subjected to phytochemical studies, and the classes of non-volatile compounds that have been reported (total number of species per genus is shown in square brackets; essential oil studies are shown in Figure 4).

\subsection{Alkaloids}

Alkaloids make up a large number of structurally diverse natural products, with more than 27,000 known compounds [2]. They typically exhibit a wide range of biological and mind-altering activities which have led to many becoming important in the pharmaceutical industry [299]. There have been approximately 244 alkaloids isolated and identified from various families of African plants, many of which exhibit antimicrobial, anti-cancer, antiinflammatory and anti-depressant-like activity [300]. Although alkaloids are relatively rare in the Lamiaceae, they may prove to be of considerable chemosystematic interest. The well-known European wood betony (Stachys officinalis (L.) Trev.) accumulates stachydrine and betonicine and it is possible that alkaloids may also occur in one or more of the 41 southern African Stachys species (or related genera of the Lamioideae). No information seems to be available, except for phytochemical screening results which suggested that four southern African Lamiaceae tested positive for alkaloids: Basilicum polystachyon (L.) Moench, Cantinoa americana (Aubl.) Harley and J.F.B. Pastore, Leonotis leonurus and Leucas matrinicensis (Jacq.) R. Br. [10,38,47,156]. The studies by Eze et al., (2013), Ladan et al., 
(2014) and Touani et al., (2014) all used the screening method documented in Harborne (1984) [301] and although potentially useful as an indicator, should be followed up by isolation and chemical characterization to confirm the presence of alkaloids. In a review by Nsuala et al. (2015) [10] on L. leonurus, the authors state that more definitive research and confirmation are needed. Similarly, it is suggested that more rigorous studies of $B$. polystachyon, C. americana and L. martinicensis are required.

\subsection{Coumarins}

The simple and versatile structure of the coumarin scaffold has made it a point of interest in many applications such as cosmetics, perfumery and the pharmaceutical industry [302]. Many coumarin compounds are appropriate contenders for modern medications as they possess strong pharmacological activity, lower drug resistance, low toxicity and side-effects, and are highly bioavailable [302]. Coumarins have been isolated and identified in several Lamiaceae species, namely lavender (Lavandula angustifolia Mill.), aspic (L. latifolia Medik.), European bulge (Lycopus europaeus L.), basil (Ocimum basilicum L.) and garden sage (Salvia officinalis) [302]. Coumarins are reported to be present in six southern African species: Acrotome inflata Benth. [34], Basilicum polystachyon [47], Cantinoa ameriacana [38], Coleus hadiensis (Forssk.) A.J. Paton [93], Marrubium vulgare L. [164] and Prunella vulgaris L. [229].

\subsection{Phenolics}

Phenolics are the second most abundant class of compounds occurring in the data, which is not surprising, as they constitute a large group of secondary metabolites in plants. Furthermore, these compounds are of great interest as nutraceuticals in the food and pharmaceutical industries [303]. Phenolic compounds have been extracted from beverages such as tea and wine, fruits and vegetables, and have undergone an immense number of studies using in vitro methods [304-308]. These studies have identified these compounds as being powerful antioxidant agents, though it has been long debated whether the same in vitro results can be obtained in vivo as the chemistry may alter after being processed by the body [304]. To overcome such issues, phenolic compounds are being loaded into nanocarriers (such as lipid-based nanocarriers, nano-emulsions, nano-scale phospholipids, and nano-structured lipid carriers) all of which are being used to mask their unpleasant flavor in oral administration, providing higher stability and absorption, and better release in gastrointestinal conditions [303]. Moreover, these formulations provide the potential for enhanced solubility, bioavailability and assist in controlled release of the nano-encapsulated phenolic compounds [303]. Apart from their health benefits, compounds such as caffeic acid esters (nepetoidin A and B) appear to be of taxonomic significance within the Lamiaceae and a wider survey may yield useful results. Nepetoidin B was first isolated from the glands of Coleus caninus (Roth) Vatke in 1975 [42].

Coleus amboinicus Lour. is a popular medicinal plant native to southern Africa, the Arabian Peninsula and India and has been used to treat a wide range of ailments, including digestive problems, skin conditions, respiratory issues, infections and pain [309]. This species is rich in phenolic compounds such as quercetin, vitexin, and coumaric-, caffeic- and cinnamic acids and exhibited very low toxicity with an $\mathrm{LC}_{50}$ value of $198.630 \mu \mathrm{g} / \mathrm{mL}$ [60]. The presence of these compounds may contribute to the use of the species as natural and traditional remedies.

Three flavonoids, hoslundin, hoslundal and hoslunddiol, as well as two pyronesubstituted flavonoids-oppositin and 5-O-methylhoslundin - have been isolated from Cameroonian Hoslundia opposita Vahl [114,116]. In a later study by Salame et al. (2012) [310], two unusual 6-furanoflavones (hoslunfuranine and 5-O-methylhoslunfuranine), along with the known methylpyranoflavonic analogues were isolated from the leaves of H. opposita. Furthermore, the antileishmanicidal capabilities of select compounds were investigated; two of which exhibited potential in the micromolar range.

In a study by El-Ansari et al. (2009) [137] on Leonotis leonurus, ten flavonoid compounds were extracted and identified in the genus for the first time, six of which were 
flavone glycosides (6-C- $\alpha$-arabinoside- $8-C-\beta$-glucoside, apigenin $8-C-\beta$-glucoside, apigenin7-O- $\beta$-glucoside, luteolin 7-O- $\beta$-glucoside, luteolin 7-O- $\beta$-glucoside- $3^{\prime}$-methyl ether and apigenin 7-O-(6"-O-p-coumaroyl)- $\beta$-glucoside), two methylated flavones (6-methoxyluteolin$4^{\prime}$-methyl ether and luteolin $3^{\prime}$-methyl ether) and two flavone aglycones (luteolin and apigenin). Furthermore, the authors investigated the hepatoprotective, anti-inflammatory and cytotoxic activities of methanol and chloroform extracts, observing that these extracts exhibited strong hepatoprotective and anti-inflammatory activity; there was however no cytotoxic activity recorded. Apigenin and luteolin are two examples of phenolic compounds extracted and identified from South African L. leonurus [10,140], and Brazilian Leonotis nepetifolia (L.) R.Br. Another study in 2015 (Oliveira et al. (2015) [148]), quantified total phenolic and flavonoid content. The extracts from L. nepetifolia exhibited a broad-spectrum of antimicrobial activity with strong action against Shigella flexneri, Enterococcus faecalis, Staphylococcus aureus, Bacillus subtilis, Helicobacter pylori and Streptococcus pyogenes attributed to the relative phenolic content.

Rosmarinic acid, luteolin-7-O-glucoside and eriocitrin have been reported as main constituents in the aqueous extract of Mentha aquatica [311]. Another study by Benabdallah et al. (2016) [166] determined a high phenolic content in Algerian provenances of M. aquatica and noted good antimicrobial activity from the methanol extract. Safaiee et al. (2019) [167] tested the extraction methods for total phenolic compounds in M. aquatica. The authors determined that freeze-drying samples, followed by extraction at $60^{\circ} \mathrm{C}$ were the most effective at obtaining a maximum yield. In 1973, eight flavonoid compounds were isolated and identified from Mentha longifolia (L.) L. namely, luteolin-7-glucoside, luteolin-7-rutinoside, luteolin-7-glucuronide, apigenin-7-glucuronide, acacetin-7-rutinoside, diosmetin-7rutinoside, hesperetin-7-rutinoside and eriodictyol-7-rutinoside [178]. In a phytochemical study of Moroccan M. longifolia, four flavonoids (5,6-dihydroxy-7,8, $3^{\prime}, 4^{\prime}$-tetramethoxyflavone, luteolin, luteolin 7-O-glucoside, and hesperidin) were isolated, all of which had previously been reported by other studies, while one $\left(5,7,4^{\prime}\right.$-trihydroxy- $6,2^{\prime}, 3^{\prime}$-trimethoxyflavone) was reported for the first time from this source [180].

The first phytochemical profiling of non-volatile metabolites in Micromeria biflora (Buch.-Ham. ex D. Don) Benth. from Kenya, produced nine flavonoids and caffeic acid oligomers, among which was a newly discovered flavone glycoside, ( $2^{\prime \prime}$-caffeoyl-luteolin 7-O- $\beta$-D-glucuronide) [191].

Seventeen of the indigenous southern African Salvia species have been investigated. Salvia aurea L. was assayed for total phenolic content and the influence that seasonal variation may have [243]. Grzeszczuk, Salachna and Meller (2018) [247] investigated the response of S. coccinea Buc'hoz ex Etl. to two concentrations of salicylic acid and increasing concentrations of sodium chloride. They noted that the application of salicylic acid relieved the effects of increased sodium chloride concentrations and subsequently resulted in increased number of branches, higher fresh herbal weight as well as higher contents of total chlorophyll, carotenoids, polyphenols and increased antioxidant activity. In a study of exudate flavonoids in some Salvia species, it was noted that S. stenophylla Burch. ex Benth. accumulated apigenin, apigenin-7-methyl ether, scutellarein-7,4'-dimethyl ether, luteolin, and 6-hydroxyluteolin-6,7-dimethyl ether. The major constituent of the leaf exudate was not identified [261]. In a study by Kamatou, Viljoen and Steenkamp (2009) [240], sixteen South African Salvia species were investigated for their phenolic content. The authors noted that compounds such as betulafolientriol oxide and rosmarinic acid were detected in all the species studied. Furthermore, carnosol, carnosic acid, oleanolic acid/ursolic acid, and rosmarinic acid were abundant in many species [240].

Several glucosides, including lamiol, lamalbid, shanzhiside methyl ester, laminoside and trace amounts of 5-deoxylaminol, sesamoside and barlerine were isolated from Lamium amplexicaule L. by Alipieva et al. (2007) [121]. The flavonoid glycoside rutin was detected in Coleus neochilus (Schltr.) Codd and C. madagascariensis (Pers.) A.Chev. [39]. Flavonoid glycosides and aglycones have been isolated and identified in Leonotis leonurus [10], Lamium galeobdolon (L.) L. [40], Mentha aquatica [175], M. longifolia [178] and Marrubium 
vulgare [164] while they have only been detected in Leucas martinicensis [156]. A rigorous survey of flavonoids in southern African Lamiaceae may possibly reveal taxonomically useful discontinuities.

\subsection{Pyrones}

$\alpha$-Pyrones have been isolated and identified in the genera Syncolostemon (four species) and Tetradenia (two species), both tribe Ocimeae. Syncolostemon argenteus N.E. Br. yielded six $\alpha$-pyrones namely, synargentolides A-E [271], while S. densiflorus Benth. gave syndenolide $[37,272]$ and S. rotundifolius had synrotolide as main compound [231].

Boronolide has been extracted from Tetradenia barberae (N.E. Br.) Codd [231,275] and in T. riparia (Hochst.) Codd, umuravumbolide (5,6-dihydro-6-(3-acetoxy-1-heptenyl)-2pyrone) and desacetylumuravumbolide was isolated for the first time in 1979, and the absolute configuration elucidated in 1995 [35,281]. Natural products belonging to the 6-substituted 5,6-dihydro- $\alpha$-pyrone family display antifungal, antimicrobial and phytotoxic activities and cytotoxicity against human tumor cells [312]. Comparative studies of geographically representative samples may lead to a better understanding of the chemical variation in Syncolostemon and Tetradenia species.

\subsection{Terpenoids and Steroids}

Terpenoids, more specifically diterpenoids, are abundant secondary metabolites present in Lamiaceae, as indicated by a large volume of data. Many species contain labdane diterpenoids, a multitude of which demonstrate a broad spectrum of biological activities, including anti-inflammatory, antimicrobial, antiviral, cytotoxic, antioxidant, antihypertensive, and hepatoprotective activities [313,314]. Apart from their functions as important biomolecules, labdane diterpenoids have potential as biomarkers for chemotaxonomic studies and chemical fingerprinting. However, the lack of chromatographic data and reference compounds limits their use in such useful applications [314]. In a review by Hussein (2018) [210], a thorough account of the diterpenoids isolated from southern African species, especially from the genera Coleus, Leonotis, Plectranthus, Salvia, and Tetradenia, is presented.

In the genus Coleus, terpenoids have been extracted from C. amboinicus, C. caninus, C. comosus Hochst. ex Gürke, C. grandidentatus (Gürke) A.J. Paton, C. hereroensis (Engl.) A.J.Paton, C. madagascariensis and C. porcatus (van Jaarsv. and P.J.D.Winter) A.J. Paton. Compounds such as coleon $\mathrm{U}$ and $\mathrm{V}$, royleanone and horminone were identified from C. grandidentatus with coleon $U$ exhibiting potent cytotoxicity against human cancer cell lines and has been deemed a promising anticancer compound needing further investigation [210]. Coleus comosus has been well studied with twenty compounds identified, eleven of which are neoclerodanes, seven labdanes and several abietanes [210]. Two abietane diterpenoids were isolated for the first time from $C$. madagascariensis ( $7 \beta$-acetoxy$6 \beta$-hydroxyroyleanone and $7 \beta, 6 \beta$-dihydroxyroyleanone), along with rosmarinic acid and coleon $\mathrm{U}$ quinone, all of which exhibited inhibitory activity on $\alpha$-glucosidase with $\mathrm{IC}_{50}$ values ranging from 33 to $275 \mu \mathrm{M}$ [102]. Furthermore, the abietane diterpenoids exhibited potent antibacterial activities against Staphylococcus aureus and Enterococcus faecalis [102].

In a study by Achenbach et al., (1992) [113] four abietane-type esters were isolated from the root bark of Hoslundia opposita, one of which (identified as 3-O-benzoylhosloppone) inhibited the growth of the multidrug resistant strain $\mathrm{K}_{1}$ of Plasmodium falciparum in vitro with an $\mathrm{IC}_{50}$ value of $0.4 \mu \mathrm{g} / \mathrm{mL}$, thus confirming its use as a traditional African remedy for the treatment of malaria [315].

Leonotis leonurus and L. nepetifolia are well studied with 21 compounds identified in L. leonurus and 30 from L. nepetifolia. Marrubiin has been isolated from L. leonurus but apparently does not occur in the other two species. Furthermore, the compounds dubiin, leonotin, leonotinin and nepetaefolin occur in L. nepetifolia and L. ocymifolia (Burm.f.) Iwarsson but has not been observed in L. leonurus. Compound ' $\mathrm{X}$ ' however, has been identified in both L. leonurus and O. ocymifolia [138,153]. 
Various classes of terpenoids have been identified from the genus Plectranthus. Seven tetracyclic phyllocladane-type terpenoids were characterized from P. ambiguus (Bolus) Codd, P. fruticosus L'Hér. yielded four labdanes and 10 kauranes, some of which exhibited moderate antimicrobial activity [210]. Plectranthus ecklonii Benth. and P. strigosus Benth. ex E. Mey. both share parviflorone D and F, both of which showed potent antibacterial activity against Listeria monocytogenes and Mycobacterium tuberculosis while parviflorone D exhibited apoptotic inducing activity in leukemia cells [210]. Other interesting terpenoids isolated from southern African Plectranthus include kauranes ent-16-kauren-19-ol and ent-16-kauren-19oic acid, which have shown antiherpetic properties [210].

Eight Salvia species have had terpenoid compounds identified. Salvia aurea and S. chamelaegnea Berg. have been documented to contain carnosol, whereas S. chamelaeagnea and S. verbenaca L. contain ursolic acid. Four nerocladanes have been identified in S. reflexa Hornem.

Only one species of Tetradenia has been investigated for terpenoids in southern Africa, namely T. riparia. It was shown to contain several terpenoids, one identified as ibozol [210]. Tetradenia is an African-endemic genus that should be further investigated.

\subsection{Volatile Oils}

Volatile oils have been extracted (mainly through steam distillation) from 63 species in 23 genera of southern African Lamiaceae. Twelve of the 23 genera have had all members investigated (mainly those with a single or a few species) and the remaining eleven genera have had oils from some members studied. Furthermore, Teucrium and Vitex are wellstudied with $67 \%$ and $60 \%$ of their species covered, compared to Salvia with only $50 \%$ of its members studied.

To reduce the complexity of the data for comparative purposes, only major compounds were listed in Table 1. Major compounds were defined as any essential oil constituent present at a level of at least $10 \%$ of the total oil composition. A total of 133 major compounds were thus identified across the 63 species. The frequency of citation of 97 of the 133 compounds (all those with three or more citations) is shown in Figure 6. $\beta$-Caryophyllene is by far the most common major compound, followed at some distance by germacrene- $D$, 1,8-cineole, limonene, $\alpha$-pinene and $\alpha$-bisabolol.

It is no surprise that $\beta$-caryophyllene is the most common major compound in the essential oil of southern African Lamiaceae as it widely distributed throughout the Plant Kingdom. It contributes to the unique aromas of essential oils and plays a pivotal role in the evolution and survival of higher plants. Furthermore, studies have provided evidence that support $\beta$-caryophyllene as a potential therapeutic tool based on the protective roles it exhibits on animal cells [316]. Moreover, experimental results have noted the ability of this molecule to reduce effects of chronic pathologies characterized by inflammation and oxidative stress, especially metabolic and neurological diseases [316]. $\beta$-Caryophyllene has exhibited beneficial effects on diabetes, cardiovascular diseases, obesity, some liver diseases, pain and other nervous system disorders [316].

Given the common occurrence of the mono- and sesquiterpenoids in several unrelated families and genera, it is not surprising to find that there are no obvious chemosystematic patterns and that essential oil is apparently produced by most if not all of the subfamilies and tribes. The presence of distinct chemotypes in genera such as Mentha and Salvia increases the complexity. Comparative studies of geographically representative samples may help to unravel patterns of diversity not previously considered, when relationships were not as well understood as they currently are.

Germacrene-D was reported as a major compound in thirteen of the sixteen genera, followed by $\beta$-caryophyllene $(9 / 16)$ and spathulenol $(6 / 16)$. Several genera with a single representative studied included Aeollanthus (A. parvifolius Benth.) with $\alpha$-muurolol as the major compound; Basilicum (B. polystachyon) with epiglobulol and yanglene; $\mathrm{Ce}$ dronella (C. canariensis Moench) with $\beta$-pinene, pinocarvone and $p$-allyl anisole; Hoslundia (H. opposita) had a single study reporting eugenol as major compound; Marrubium (M. vulgare) $\gamma$-eudesmol and $\beta$-caryophyllene; Mircomeria (M. biflora) exhibiting geranial, neral, 
germacrene-D and linalool; Orthosiphon (O. thymiflorus (Roth) Sleesen) has 2-isopropyl-5methyl-9-methylene-bicyclo-1-decene(4.4.0); Platostoma (P. rotundifolium (Briq.) A.J.Paton) was reported have germacrene-D, $\beta$-caryophyllene and spathulenol; Prunella (P. vulgaris) germacrene-D and aromadendrene; and Tetradenia (T. riparia) having fenchone as the most reported major compound.

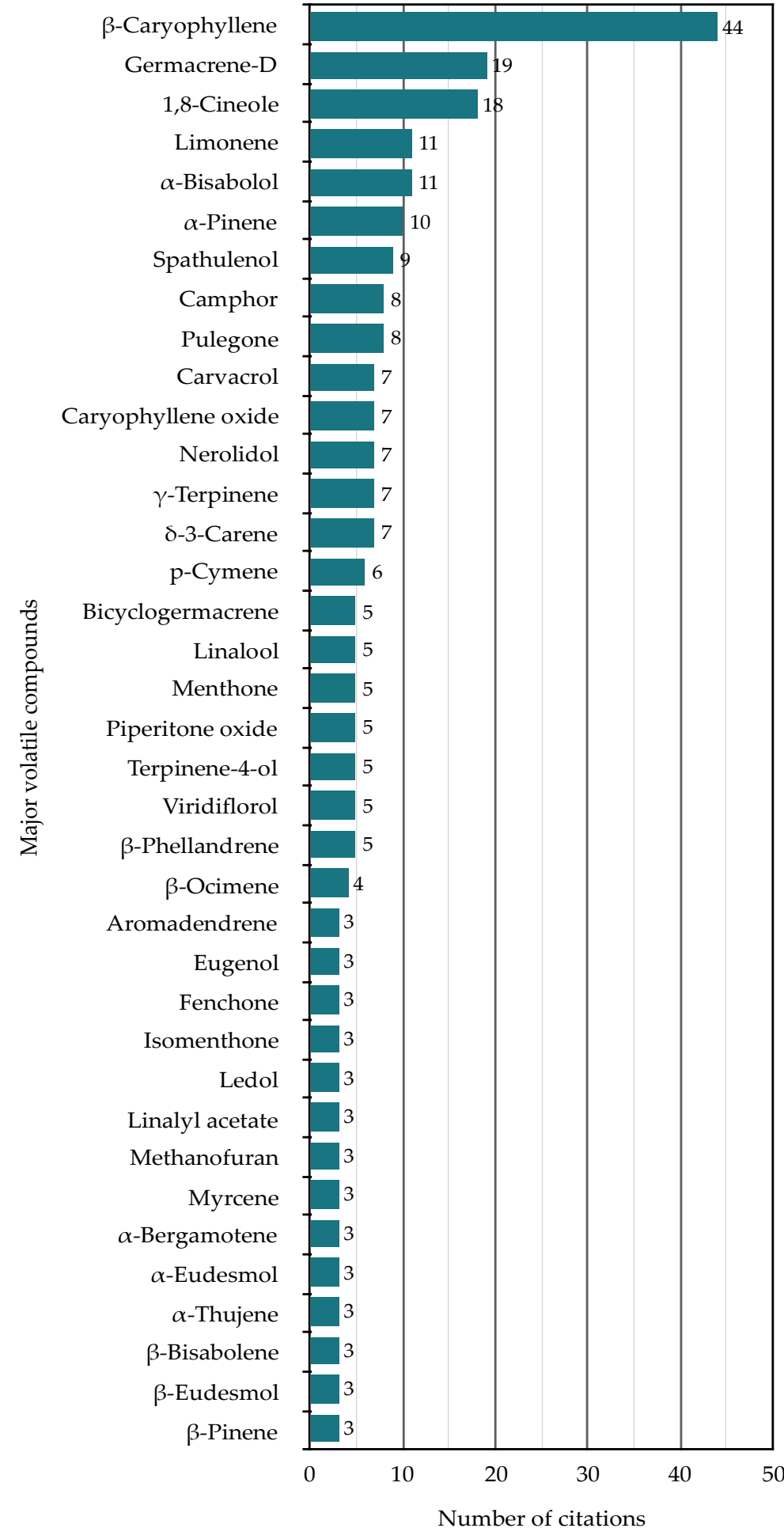

Figure 6. Summary of the frequency of citation of major essential oil compounds (i.e., those representing more than $10 \%$ of the composition of the oil) reported from the genera of southern African Lamiaceae. Only compounds with three or more citations are shown. 
Studies on both species of Cantinoa (C. americana and C. mutabilis (Rich.) Harley and J.F.B. Pastore) documented $\beta$-caryophyllene in both species. However, several studies of C. mutabilis reported different major compounds, namely germacrene-D, 1,8-cineole, limonene, spathulenol, and camphor. Coleus had eight of its members investigated with C. caninus, C. comosus, and C. madagascariensis documented as having $\beta$-caryophyllene as one of their major compounds. Carvacrol and caryophyllene were frequently reported compounds in C. amboinicus, and camphor in C. grandidentatus. Lamium amplexicaule was documented to have camphor and germacrene-D as the major compounds.

For Leonotis, germacrene-D and $\beta$-caryophyllene were frequently reported for all species within the genus. Leonotis leonurus was also recorded to have limonene and $\alpha$ pinene as additional major compounds. In Mentha, major compounds were pulegone and menthone, with $M$. aquatica being the only member containing $\beta$-caryophyllene as a major compound. Both M. aquatica and M. longifolia contained 1,8-cineole in a notable proportion. For Ocimum, O. americanum L. it was reported to have 1,8-cineole as the major compound in several studies, followed by camphor, while O. gratissimum L. had germacrene-D and eugenol, as reported in a single study. Plectranthus, sensu stricto had only two species investigated, with spathulenol and bicyclogermacrene as major compounds, and sabinyl acetate in P. fruticosus.

$\beta$-Caryophyllene was noted as the most cited major compound in Salvia, recorded in seven of the eighteen species studied. For three closely related Salvia species (S. repens Burch. ex Benth., S. runcinata L.f. and S. stenophylla), $\alpha$-bisabolol was recorded frequently, indicating at least some chemosystematic value. Other major compounds documented for Salvia included 1,8-cineole, limonene, and $\alpha$-pinene. One study recorded $\alpha$-cubebene and $\beta$-cubebene as major compounds in both Teucrium africanum Thunb. and T. sessiliflorum Benth. Vitex was shown to have three species exhibiting 1,8-cineole as one of their major compounds.

In order to make progress in chemosystematic evaluations of essential oil compounds, multiple samples will be essential and multivariate statistical approaches are most likely needed to detect less obvious patterns. It may also be useful to explore the biosynthetic relationships between the individual compounds to ascertain the possible presence or absence of particular biosynthetic pathways that can be used to support or disprove relationships.

\section{Traditional Uses}

Throughout history, there have been numerous accounts of Lamiaceae species used in traditional (folk) medicines and as functional foods. This is likely due to the preserved knowledge regarding their benefits and effects exhibited by their preventative and curative properties. Many ethnic groups across the globe have at least a single recorded account of Lamiaceae as either a cure for an ailment, or the flavoring and preservation of food. Many species native to Europe have been used as culinary herbs and spices, including rosemary, sage and thyme. Their use is not only limited to medicines and food, but also form part of ceremonies, rituals and 'magic' — such as the use of Salvia apiana Jeps. (white sage) by the Native Americans and Ocimum sanctum L. (holy basil) in India for prayers and rituals [317,318]. A study in Lebanon documented, for the first time, several genera (Lavandula L., Melissa L., Mentha, Origanum L., Rosmarinus L., Salvia, Satureja and Thymus L.) used as curatives against gastro-intestinal disorders, hypoglycemia, respiratory disorders, and as cardiotonics and antihypertensives, to name but a few [319]. In China, species such as Scutellaria baicalensis Georgi, Salvia militiorrhiza Bunge, Clerodendrum bungei, and Leonurus japonicus Houtt. have well-documented histories in Traditional Chinese Medicine [320-322]. It should not be overlooked that the use of local wild resources, in this case the Lamiaceae, must be supported by concepts such as the "ecological economy", "sustainable development" and "equitable benefit-sharing" [323].

Southern Africa is no different with a wide array of recorded traditional uses, as summarized in Figure 7 (a list of publications used to generate the figure are listed in Supplementary Materials Table S2). Some of these are briefly discussed below. 


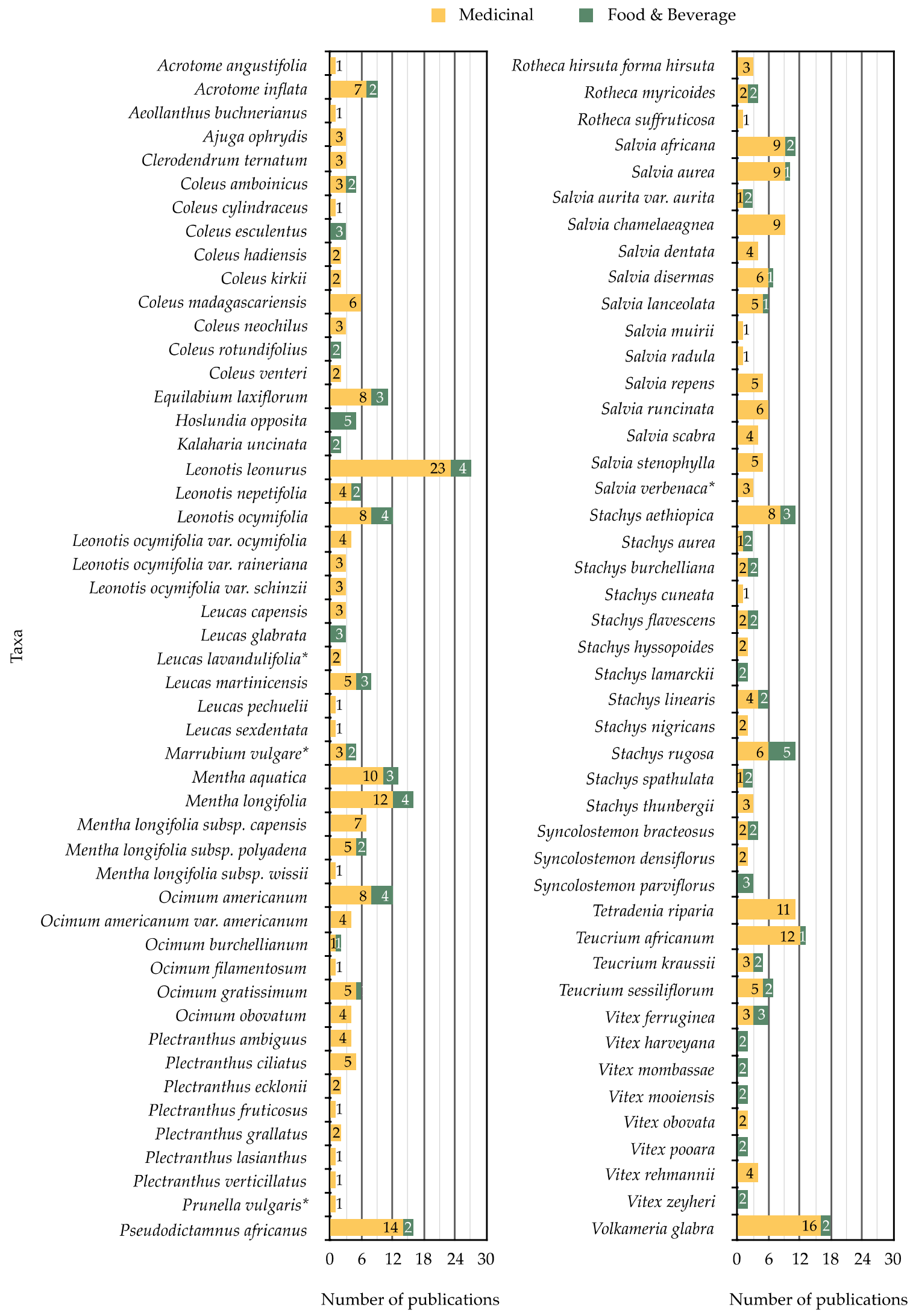

Figure 7. The numbers of publications where medicinal (yellow) and food and beverage (green) uses have been recorded for southern African genera and species of Lamiaceae. 


\subsection{Medicinal Uses}

A total of 83 taxa have documented medicinal uses in southern Africa (Figure 7). The genera with the highest number of records include Salvia (68 records), Leonotis (45 records), Mentha (35 records), Stachys (32 records) and Ocimum (23 records) (Figure 7). Well-documented species of medicinal value include Leonotis leonurus (23 records), Volkameria glabra (E.Mey.) Mabb. and Y.W.Yuan (16 records), Pseudodictamnus africanus (14 records), Mentha longifolia (12 records), Teucrium africanum (12 records) and Tetradenia riparia (11 records) (Figure 7). Several of these species are noted by Van Wyk et al. (2009) [15] as being part of the 150 best known and most popular herbal medicines in South Africa (Figure 1).

Other species such as Acrotome angustifolia G.Taylor is used as a medicinal tea administered to children for upset stomachs whereas A. inflata is used in Namibia as a treatment for coughs, fever and breast pain, and in the Eastern Cape of South Africa, it used as a treatment for skin ailments, including chickenpox, wounds, sores, rashes and eczema $[17,324,325]$. The use of $A$. inflata for topical use is corroborated by Iyambo, Kibuule and Ilonga (2017) [34] who tested methanolic and aqueous extracts of the plant on several bacterial strains, including Pseudomonas aeruginosa, Staphylococcus aureus and Bacillus subtilis. The results produced in the study were comparable with that of penicillin, although the extracts did not show any activity as an antifungal agent against Candida albicans. Furthermore, A. inflata is used in a multi-ingredient remedy 'Sehlare se Seebana' by the northern Sotho as a treatment for epilepsy. In a study by Jäger et al. (2005) [326], aqueous and methanol extracts of $A$. inflata, along with five other plant species, were screened using the $\mathrm{GABA}_{\mathrm{A}}$-benzodiazepine binding assay. Two of the plant species exhibited good dose-dependent activity, although A. inflata was not one of them. Aqueous and ethanol extracts were prepared for the six species together and tested, it was noted that the ethanol extract for the mixture was more active than the aqueous one, though the results did not suggest any synergistic effects.

Southern Sotho people administer a traditional medicine made from Aeollanthus buchnerianus Briq. as a cold remedy for children, and the use of Ajuga ophrydis Burch. ex Benth. to treat reproductive issues such as sterility and infertility is also well documented $[327,328]$. Compounds isolated from A. buchnerianus have been documented to exhibit in vitro antifungal properties against Cladosporium cucumerinum, Aspergillus niger and Pythium ultimum, as well as antimicrobial activity against $B$. subtilis, $S$. aureus and Streptomyces scabies with MIC values of 80,20 and $20 \mu \mathrm{g} / \mathrm{mL}$ respectively [30].

In north-eastern Namibia, Clerodendrum ternatum Schinz is documented as a remedy for urinary problems as well as a topical treatment for leg ailments, and in South Africa it has been used by the Bapedi people to treat chronic cough [329,330].

From the genus Coleus, $C$. amboinicus has been used medicinally for both humans and livestock [331]. In a study by Sahrial and Solfaine (2019) [332], the authors noted that the ethanolic extract of $C$. amboinicus administered to Wistar rats with cisplatin-induced nephropathy inhibited pathological lesions by regulating the renal expression of TGF- $1 \beta$ in areas containing renal glomeruli and tubules. Furthermore, C. amboinicus has shown to exhibit moderate to high antibacterial $[66,67,333,334]$, antioxidant/cytoprotective $[333,335,336]$, and anticancer $[336,337]$ activity.

The Zulu people have used $C$. hadiensis for treating chest ailments such as coughs, C. kirkii as a mouthwash for aching teeth caused by neuralgia, and C. madagascariensis for respiratory ailments and topical use [338]. Coleus hadiensis has been reported to exhibit moderate antimicrobial activity against Sclerotinia sclerotiorum, Candida species, B. subtilis and Xanthomonas campestris producing inhibition zones of $16 \mathrm{~mm}, 15 \mathrm{~mm}, 8 \mathrm{~mm}$ and $10 \mathrm{~mm}$ respectively [96]. Another study by Menon, Sasikumar and Latha (2011) [339], in vitro anti-inflammatory and cytotoxic activity of $C$. hadiensis were studied. The authors noted that the plant extract at a concentration of $1 \mathrm{mg} / \mathrm{mL}$ exhibited $86.10 \%$ BSA denaturation inhibition, $87.49 \%$ platelet aggregation inhibition and $87.26 \%$ HRBC membrane stabilization, results similar to the standard non-steroidal drug, Dicoflenac. Furthermore, an $\mathrm{IC}_{50}$ value of $141.3 \mu \mathrm{g} / \mathrm{mL}$ was recorded for the cytotoxicity against HeLa cells, sug- 
gesting that the methanolic extract of $C$. hadiensis has promising therapeutic potential. For C. madagascariensis bactericidal activity against B. subtilis, Micrococcus species, S. aureus, and Yersinia enterocolitica have been recorded, and also revealed to show modest antioxidant activity [101]. In another study, the acetone extract of $C$. madagascariensis exhibited potent antibacterial activity against Gram-positive bacteria with MIC values ranging from 1.95 to $7.81 \mu \mathrm{g} / \mathrm{mL}$, and moderate activity against Gram-negative bacteria with MIC values ranging from 0.48 to $3.91 \mu \mathrm{g} / \mathrm{mL}$ [39]. Moreover, potent antioxidant activity was noted, and moderate cytotoxicity was recorded against triple negative human breast carcinoma [39]. Coleus venteri (van Jaarsv. and Hankey) A.J.Paton has been documented for treating influenza, blocked nasal passages and high blood pressure in Central Sekhukhuneland, South Africa [340,341]. A study by Maree et al., (2014) [111] investigated the inhibitory activities of two bioactive acetophenones from $C$. venteri, where the authors noted marked inhibitory activities against the transfer of the IncW plasmid R7K in a bacterial plasmid transfer inhibition assay.

Equilabium laxiflorum (Benth.) Mwany. and A.J. Paton has been documented to treat a wide range of ailments including troubled eyes, colds, influenza, stomach upset, bleeding gums and fever [338,342,343]. A study by Maharaj et al. (2010) [344] screened the two-minute mosquito repellency of aqueous and organic extracts of South African medicinal plants in a rodent model; E. laxiflorum being one of them. The authors noted $55 \%, 53 \%$ and $38 \%$ repellency for aqueous, organic and 1:1 aqueous-organic E. laxiflorum extracts respectively.

Recorded medicinal uses of Hoslundia opposita include the treatment of cystitis, liver disease, stomach ailments, gonorrhea, eye concerns and snakebites [345]. The acute toxicity of the ethanolic extract of H. opposita was investigated by Oloyede and Akindele (2020) [346] in Swiss mice (Mus musculus). The study concluded that $H$. opposita is devoid of acute toxicosis at the examined doses of 50, 100 and $200 \mathrm{mg} / \mathrm{kg}$ over a period of fourteen days. Other studies have investigated the central nervous system depressant activity of $H$. opposita. A study by Olajide, Awe and Makinde (1999) [347] observed that the chloroform extract of $H$. opposita significantly enhanced the phenobarbitone sleeping time in mice and produced a $60 \%$ protection against leptazol-induced convulsions. Another study by Risa et al. (2004) [348] noted the ethanolic leaf extract of H. opposita, among several other plant extracts, to be the most active against epilepsy and convulsions using the $\mathrm{GABA}_{\mathrm{A}}$-benzodiazepine receptor assay.

The genus Leonotis has many documented medicinal uses. The crushed leaves of L. leonurus are used to prepare a decoction which is taken orally twice daily to assist in weight-loss and as an anti-diabetic aid [349-351]. Furthermore, it has been documented to treat cancer, ulcers, gout, as well as aches and pains [11]. The pharmacology of L. leonurus is well-recorded as reviewed by Mazimba (2015) [140] and Nsuala et al. (2015) [10], though some notable studies include the in vitro anti-HIV activity of several South African medicinal plant extracts, of which L. leonurus was recorded to exhibit significant HIV-1 inhibition (33\% reduction in HIV-1 p24, $p<0.05$ ) [352]. Other studies have observed noteworthy anti-inflammatory and analgesic activity [137], as well as anthelmintic [353,354], hepatoprotective [137] and anti-diabetic activities [355]. Furthermore, the presence of marrubiin in several Lamiaceae species and the pharmacological efficacy of this terpenoid has been well documented. Several studies and reviews illustrated the effect of L. leonurus extracts as cardioprotective, antidiabetic, gastroprotective, antispasmodic and analgesic agents [10,356-358]. Leonotis nepetifolia has been used to treat influenza, chest conditions, insect stings and snake bites, whereas L. ocymifolia and its varieties have documented uses to treat high blood pressure, poor blood circulation, rheumatism, diabetes, nerve weakness and snakebites [359-361]. A study on Brazilian L. nepetifolia by de Oliveira et al. (2019) [362], noted potent antileishmanial activity from leaf and root extracts (IC $\mathrm{C}_{50}$ values of $32.90 \mu \mathrm{g} / \mathrm{mL}$ and $57.70 \mu \mathrm{g} / \mathrm{mL}$ respectively), while leaf extracts inhibited Bacillus cereus $(125 \mu \mathrm{g} / \mathrm{mL})$, and S. aureus $(100 \mu \mathrm{g} / \mathrm{mL})$, as well as exhibiting anti-Candida activity ( $\mathrm{IC}_{50}$ values ranging from 10-125 $\mu \mathrm{g} / \mathrm{mL}$ ). A study by Oyedeji and Afolayan (2005) [129] 
on the antimicrobial activity of both L. leonurus and L. ocymifolia from the Eastern Cape, revealed that both species exhibited a broad spectrum of antimicrobial activity against both Gram-positive and Gram-negative microorganisms with MIC values ranging from $0.039-1.25 \mathrm{mg} / \mathrm{mL}$.

Leucas capensis (Benth.) Engl. has been used for oral hygiene, headaches, sore eyes, and a treatment for hemorrhoids and chest ailments [17,327,363]. Leucas lavandulifolia Sm., L. martinicensis, L. pechuelii (Kuntze) Baker and L. sexdentata Skan have been used to treat conditions such as fever, aches, pains, respiratory ailments, stomach cramps, dizziness, and gastro-intestinal and skin ailments [345,364,365]. Leucas lavandulifolia has shown to exhibit significant in vivo antidiabetic, antioxidant and hepatoprotective activities in rats [366,367], while L. martinicensis has been reported as having notable antioxidant activity [368] although extracts are relatively safe, prolonged use carry the risk of cardiac toxicities [156].

The naturalized exotic, Marrubium vulgare, has been documented to treat respiratory conditions, fever and inflammation [343,369,370]. In reviews by Lodhi et al. (2017) [371] and Yabrir (2019) [372], the authors provide an in-depth view of the pharmacological activity of $M$. vulgare which include analgesic, anti-inflammatory, anti-spasmodic, immunomodulatory, antimicrobial and cytotoxic activities, to mention a few.

Mentha longifolia is used as a treatment for coughs, colds, asthma and other respiratory ailments [13]. A tea is prepared from the leaves and said to alleviate colds and $\mathrm{flu}$, as well assist with stomach problems. The warm leaves as used as a compress to treat headaches [373,374]. In a study in 1997, McGaw, Jäger and Van Staden (1997) [375] investigated the ability of 26 South African traditional medicines to inhibit prostaglandin synthesis. Of the species investigated, $M$. longifolia exhibited moderate to higher activity (52-91\%) with increasing amount of extract (50-100 $\mu \mathrm{g})$; the organic extract had the higher potential of the extracts tested. A study from Iraq investigated the antimicrobial activity of $M$. longifolia based on its use as a folk remedy for sore throats and oral irritations. Menthone was isolated and identified as the antibacterial compound which exhibited excellent antimicrobial activity against several clinical pathogens, thus validating the plants use in the treatment for oral concerns [376]. Similarly, M. longifolia from Algeria presented comparable results where the organic extracts displayed favorable antimicrobial activity against several pathogens which as attributed to the high phenolic content [377].

In the genus Ocimum, the highly aromatic O. americanum has been used both cosmetically (as a perfume powder), topically (for burns and wounds) and orally as a medicine for chest complaints such as asthma $[329,343,378]$. The smoke is said to be inhaled as a remedy to stop nosebleeds [379]. Ocimum burchellianum Benth. and O. gratissimum have documented uses as medicinal teas, while O. filamentosum Forssk. and O. obovatum E.Mey. ex Benth. have both been used as a treatment to aid hair growth $[17,338,343,361]$. The volatile oil of $O$. americanum exhibited excellent antimicrobial activity against several pathogens, including E. faecalis, Enterococcus faecium, Proteus vulgaris, S. aureus and S. epidermis [380]. In a study by Cavalcanti et al. (2004) [381], both O. americanum and O. gratissimum exhibited excellent larvicidal activity against Aedes aegypti with $\mathrm{LC}_{50}$ values of 67 and $60 \mathrm{ppm}$ respectively, thus suggesting their potential use for the control of mosquitos. The antimicrobial activity of $O$. obovatum ethanol extract reported excellent activity against $B$. subtilis with an MIC value of $0.074 \mathrm{mg} / \mathrm{mL}$, indicating its use to treat gastro-intestinal ailments may indeed be effective [382].

Plectranthus has several species with documented accounts of medicinal use. Plectranthus ambiguus has been used by the Zulu people as a treatment for skin sores, chest complaints, tonsilitis, coughs, fever and eye problems [364]. Plectranthus ciliatus E. Mey. has been used as both an analgesic, ophthalmic and a soap-substitute to wash sheep skins; P. grallatus Briq. has been used in a similar manner [327,359,383]. Antimicrobial studies conducted on P. ciliatus presented overall poor activity on test organisms, although moderate activity was recorded against $S$. sclerotiorum and B. cereus $[91,96]$. Plectranthus ecklonii 
exhibited excellent antimicrobial activity against Streptococcus sobrinus and S. mutans with MIC values of 4.7 and $5.0 \mathrm{mg} / \mathrm{mL}$ respectively [384].

Rotheca hirsuta (Hochst.) R. Fern. has documented use as a treatment for intestinal worms, urinary infections and scrofula swellings [328,331,338], while both $R$. myricoides (Hochst.) Steane and Mabb. and R. suffruticosa (Gürke) Verdc. are said to be used as treatment for snakebites [338,385]. Rotheca myricoides has been reported to exhibit excellent in vitro antimicrobial activity against methicillin-resistant S. aureus, S. aureus, Escherichia coli, Shigella sonnei, C. albicans and Mycobacterium tuberculosis [386]. Other studies have reported that the freeze-dried extracts of $R$. myricoides possessed potent antihyperglycemic and antidyslipidemic effects in vivo [387], though prolonged treatment with R. myricoides extracts in mice have reported to cause reduction in body weight, damage to kidneys and liver, and changes in some hematological and biochemical parameters [388].

Pseudodictamnus africanus (=Ballota africana (L.) Benth.), known locally as 'kattekruie', is used as a treatment for stomach and heart problems, and is said to improve blood circulation [11]. Other documented uses include the treatment of fever, measles, colds and flu, asthma, bronchitis, headaches and hysteria [15,389]. Pseudodictamnus africanus displayed excellent antimicrobial activity against respiratory pathogens (Streptococcus pyogens, Klebsiella pneumoniae and S. aureus) and as an antifungal agent against C. albicans. Furthermore, extracts of the plant has exhibited $80-100 \%$ affinity for histamine binding, thus making it an excellent antihistamine [390]. Cock and Van Vuuren (2014) [391] screened thirteen South African plant species for their antimicrobial activity against K. pneumoniae and its subsequent cause of inflammation. They found among others, that P. africanus displayed K. pneumoniae inhibition with MIC values below $1000 \mu \mathrm{g} / \mathrm{mL}$.

Salvia africana has several accounts as a remedy for colds, coughs, menstrual complaints and diarrhea $[11,389,392]$. This species has been documented to exhibit high antioxidant activity and showed potential as an anticancer agent against breast cancer cells [236]. Furthermore, antimicrobial studies using the essential oil have indicated fair to moderate activity $[237,238]$. Other Salvia species such as S. aurea, S. aurita L.f., S. chamelaeagnea, S. dentata Aiton, S. disermas L. and S. lanceolata Lam. are used to treat respiratory ailments, coughs, influenza, fever, and inflammation [351,359,393,394]. Salvia repens and S. runcinata are used topically to treat burns, sores and dermatological conditions, whereas S. scabra Thunb. is documented as a remedy for pediatric conditions [327,351,395]. An antimicrobial study on the volatile oils of $S$. aurea, S. aurita, S. chamelaegena, S. disermas, S. lanceolata, S. repens and S. runcinata exhibited moderate activity against E. coli, K. pneumoniae, B. cereus and S. aureus with MIC values ranging from 0.03 to $6.00 \mathrm{mg} / \mathrm{mL}$, while moderate antimycobacterial activity with an MIC value of $0.50 \mathrm{mg} / \mathrm{mL}$ was recorded for all oils [244]. Another study by Kamatou, Viljoen and Steenkamp (2009) [240] investigated the antioxidant and anti-inflammatory activities of sixteen South African Salvia species. The authors noted that majority of the extracts displayed antioxidant activity ( $\mathrm{IC}_{50}$ values ranging from 1.6 to $74.5 \mu \mathrm{g} / \mathrm{mL}$ and 11.9 to $69.3 \mu \mathrm{g} / \mathrm{mL}$ by means of the 2,2-diphenyl-1-picrylhydrazyl and 2,2'-azino-bis (3-ethylbenzothiazoline-6-sulfonic acid) scavenging assays), while fifteen of the extracts displayed poor anti-inflammatory activity (all $\mathrm{IC}_{50}$ values greater than $100 \mu \mathrm{g} / \mathrm{mL}$ with the exception of $S$. radula which presented an $\mathrm{IC}_{50}$ value of $78.8 \mu \mathrm{g} / \mathrm{mL}$ ) using the 5-lipoxygenase assay [240].

Though mainly used as a tea, the genus Stachys has several medicinal accounts. Stachys aethiopica L. is used topically to clean and disinfect wounds, as well as to treat respiratory ailments, colds, bronchitis and influenza [351]. Stachys aurea Benth., S. burchelliana Launert, S. flavescens Benth., and S. hyssopoides Burch. ex Benth. are used for chest and respiratory ailments $[17,378,393,396]$; while S. linearis Burch. ex Benth. and S. rugosa Aiton are used both as a lactogogue and as a lotion for wounds $[343,393]$.

The leaves of Tetradenia riparia are highly aromatic and are used throughout Africa to treat coughs, sore throats, fever, boils, mumps, malaria and dengue fever [268,364,397]. In southern Africa however, strong focus as a topical treatment has been documented due to its wound-healing and dermatological capabilities. The organic and aqueous extracts 
have indicated moderate to strong activity against bacteria and fungi [398-402]. In other studies, the efficacy of $T$. riparia as a treatment for malaria have been investigated with extracts demonstrating moderate anti-malarial activity against two strains of Plasmodium falciparum [403].

The genus Teucrium have been used ethnopharmacologically for centuries as treatments for ailments ranging from gastrointestinal disorders, respiratory ailments, inflammation and rheumatism [404], documented in both European and southern African literature. Three southern African-endemic species, T. africanum, T. kraussii Codd and T. sessiliflorum have been recorded as a treatment for stomach ailments, colds and flu, snakebites, prostate problems, sore throats, indigestion and as a general tonic for good health $[15,289,351,389]$. In ethnoveterinary medicine, T. africanum is used against gall sickness in cattle, heartworm and bloating in livestock such as goats and sheep [405]. All three species have been investigated for antimicrobial activity with T. africanum showing some activity against Escherichia coli (with an MIC value of $0.13 \mathrm{mg} / \mathrm{mL}$ ), T. kraussii against $S$. pyogenes (with an MIC value of $0.8 \mathrm{mg} / \mathrm{mL}$ ) and T. sessiliflorum exhibiting activity against $P$. aerginosa (with an MIC value of $0.5 \mathrm{mg} / \mathrm{mL}$ ) $[289,406]$.

Volkameria glabra, the only representative of this genus, has been used to treat a variety of ailments. The Zulu people use it as a treatment for internal worms and parasites, coughs, fevers, respiratory and circulatory issues, as well as gastro-intestinal and reproductive ailments $[338,364]$. Other documented uses include the treatment of snakebites and skin irritations, and the cleaning of wounds $[343,365,385,407]$. Extracts of $V$. glabra have shown to exhibit anti-bacterial, anti-fungal, anti-leishmanial and anti-plasmodial activity [408-412].

The in vitro and in vivo pharmacological activities noted by various studies for many southern African Lamiaceae further corroborate their use as natural remedies for the treatment of a wide range of ailments.

\subsection{Food Uses}

Africa has contributed several crops used as food sources worldwide. In Kunkel (1983) [413] the author accounts for ca. 12,000 plant species considered edible by humans, many of which are native to Africa and include species such as coffee (Coffea arabica L.), yams (genus Dioscorea Plum. ex L.) and African oil palm (Elaeis guineensis Jacq.). In Ethiopia, several Lamiaceae species are used as food sources, including Leucas calostachys Oliv. (as a famine food), Salvia dianthera Roth ex Roem. and Schult (as a vegetable) and Mentha aquatica (as a culinary herb) [414].

\subsubsection{Fruits and Vegetables}

Forty-five (45) species have documented uses as being edible [415-425] with H. opposita (five records), L. leonurus (four records), and several Vitex species (13 records) having the highest number of citations.

The stem tubers of Coleus esculentus and the root tubers of $C$. rotundifolius are excellent sources of nutrients and were probably once major sources of starch but they have largely been replaced by the introduction of high-yielding potatoes and sweet potatoes. Coleus esculentus is a highly adaptable species and can grow in almost any climatic zone, provided rainfall is moderate and soil has good drainage. Furthermore, the plant's proclivity to survive difficult conditions make it an excellent candidate as an alternative crop [415-418]. The sugary fruits of Hoslundia opposita, Vitex ferruginea Schumach. and Thonn., V. harveyana H. Pearson, V. mombassae Vatke and V. mooiensis H. Pearson are enjoyed as a snack by local cultural groups. The leaves of L. leonurus are sometimes used as a leafy vegetable substitute or spinach $[23,418,419]$. Other species such as Mentha aquatica, M. longifolia and Ocimum americanum are used as flavorants or condiments with meals [363,418].

\subsubsection{Beverages}

Stachys, Mentha and Salvia had the most documented uses as beverage plants, with twenty, nine and seven records respectively. Generally, hot infusions are prepared from 
Mentha aquatica, M. longifolia, Ocimum americanum, Salvia africana and several Stachys species, as likely alternatives to coffee or tea $[14,418,419]$. In some instances, $M$. longifolia is used to flavor water, as documented by Hulley (2018) [363]. The traditional use of several species of Stachys as teas and tonic in southern Africa and the obvious lack of chemical information on these species represent a significant knowledge gap.

\subsection{Other Uses}

The aromatic characteristic that many Lamiaceae species possess make then useful as insect repellents and deterrents. On some occasions, plants are burnt in huts, to fumigate after an illness and to ward off unwanted insects. The Sotho people have used Salvia repens, S. runcinata and S. stenophylla for this purpose [328]. Coleus neochilus and C. cylindraceus (Hochst. ex Benth.) A.J. Paton have been used as deterrents for flies, mosquitos, and snakes. They are either planted around the homestead, or crushed leaves are placed inside the house $[338,341,359]$. The highly aromatic leaves of Ocimum americanum and O. gratissimum have been used by local people as a perfume powder for the body, and as an insect repellent $[359,378]$.

\section{Discussion}

This review shows that some progress has been made towards unravelling the chemical diversity in southern African Lamiaceae but also revealed that several genera and many species remain to be studied. Our hypothesis that the southern African Lamiaceae have remained scientifically poorly explored can therefore not be rejected. Considerable progress is likely to be made by using modern phytochemical methods, such as liquid chromatography-mass spectrometry and metabolomics. Such an approach will not only serve to rapidly extend the coverage of taxa, but also to re-examine even those species that were apparently well studied using classical methods.

Once a more complete picture of the chemical diversity in medicinal species emerges, it will be rewarding to relate the biological activities of individual compounds to the ailments treated, and to explain the popularity and rationale behind the traditional remedies. Several species are used as teas and tonics (especially in the chemically poorly known southern African species of Stachys), suggesting potential as new functional food products. It is likely that individual compounds act in an additive or even synergistic way, which complicates the way in which pharmacological studies can be approached. Given their chemical complexity, it is also likely that different compounds or classes of compounds in the Lamiaceae act on different organ systems, resulting in the maintenance and improvement of health. Unlike pure chemical entities used in most modern pharmaceutical products, some compounds in chemically diverse herbal products may act on the respiratory system, others on the gastro-intestinal system and some perhaps directly on the brain, to generate or improve a sense of well-being. One of the first studies to demonstrate powerful antimicrobial synergy [426] was the combination of camphor and 1,8-cineole as main compounds in Osmitopsis asteriscoides (L.) Cass. (Asteraceae), a popular traditional medicine used in Cape Herbal Medicine [389]. It is likely that our understanding of the efficacy of traditional medicines will increase once their chemical composition and associated pharmacological activities become better known. Our review can be used as a starting point for future explorations of the taxonomy, chemosystematics, chemistry and pharmacological activity of southern African Lamiaceae, especially to fill in the obvious gaps in our knowledge of hitherto poorly studied taxa.

\section{Materials and Methods}

Species data was collected from Codd (1985) [17] and the South African National Biodiversity Institute's 'Plants of southern Africa' website (www.newposa.sanbi.org). Scientific names and synonyms were validated through Kew's 'Plants of the World' database (www.plantsoftheworldonline.org) as it was found to be the most up-to-date database. 
Literature searches were conducted by searching several scientific electronic databases, including GoogleScholar (www.scholar.google.com), EBSCOhost (www.ebsco.com), PubMed (www.pubmed.ncbi.nlm.nih.gov), SciFinder (www.scifinder.cas.org), ScienceDirect (www.sciencedirect.com), Springer (www.springer.com) and Wiley Online Library (www.onlinelibrary.wiley.com). Key words were used to search for literature, and this was conducted in the following manner: ("Species name" AND "synonyms" AND "chem*"), ("Species name" AND "synonyms" AND "med") and ("Species name" AND "synonyms" AND "traditional use"). A collection of scientific papers, books, dissertations and theses, and unpublished sources were compiled.

Occurrence data were downloaded from the 'Global Biodiversity Information Facility' (www.GBIF.org (accessed on 16 May 2021)) GBIF Occurrence Download (https:/ / doi.org/ 10.15468/dl.b8zqjh) and distribution maps generated in QGIS (QGIS Development Team (2021)). QGIS Geographic Information System. Open-Source Geospatial Foundation Project (http:/ / qgis.osgeo.org).

Data was cleaned, sorted analyzed in Microsoft Excel and the graphs were generated in Apple Numbers.

\section{Conclusions}

Lamiaceae is undoubtedly an important and diverse plant family not only globally, but in southern Africa as well. The phytochemical and aromatic diversity have made members of this family of potential value to the food, beverage, cosmetic and pharmaceutical industries. The popular use of Lamiaceae species as traditional medicines and food sources span across the globe and their uses are supported by scientific research. The largely unstudied diversity of chemical constituents and volatile oils of southern African Lamiaceae offer many potential applications as new functional foods and herbal remedies. Southern Africa holds an abundance of opportunities for further research within this diverse and commercially relevant group, with respectively $66 \%$ and $71 \%$ of its species yet to be explored chemically and ethnobotanically.

It is likely that our understanding of the efficacy of traditional medicines will increase once their chemical composition and associated pharmacological activities become better known. Our review provides clarity on the current state of knowledge on the taxonomy and chemistry of southern African Lamiaceae and associated key publications and will hopefully serve as a useful framework to guide future phytochemical and ethnopharmacological studies.

Supplementary Materials: The following are available online, Table S1: List of all southern African Lamiaceae (naturalized non-indigenous species are indicated with an asterisk), Table S2. Publications consulted for ethnobotanical data.

Author Contributions: The concept for this paper developed as an invited lecture (by B.-E.V.W.) at the International Symposium: Advances in Lamiaceae Science, 26-29 April 2017, Antalya, Turkey. R.D.R. conducted most of the data gathering and both authors wrote the paper. Both authors have read and agreed to the published version of the manuscript.

Funding: Research was funded by the University of Johannesburg and the National Research Foundation of South Africa (to the National Research Chair in Indigenous Plant Use, NRF grant numbers 84442 and MND200626537046).

Data Availability Statement: The geographical data presented in this study are openly available in GBIF (Global Biodiversity Information Facility) at https:/ / doi.org/10.15468/dl.b8zqjh (accessed on 16 May 2021).

Acknowledgments: K. Hüsnü Can Başer and his colleagues, Gulmira and Temel Özek, are thanked for collaborative studies on the essential oils of medicinal plants over a period of many years. B.-E.V.W. also thanks Başer for the invitation to attend the International Lamiaceae symposium in Antalya, Turkey, in 2017. The authors thank three anonymous reviewers for suggestions which improved the manuscript. 
Conflicts of Interest: The authors declare no conflict of interest. The funders had no role in the design of the study; in the collection, analyses, or interpretation of data; in the writing of the manuscript, or in the decision to publish the results.

\section{References}

1. Prakash, O.; Chandra, M.; Pant, A.K.; Rawat, D.S. Mint (Mentha spicata L.) Oils; Elsevier Inc.: Amsterdam, The Netherlands, 2016; ISBN 9780124166448.

2. Van Wyk, B.-E.; Wink, M. Medicinal Plants of the World; CABI: Boston, MA, USA, 2017; ISBN 1786393255.

3. Van Wyk, B.-E. Food Plants of the World: Identification, Culinary Uses and Nutritional Value; CABI: Boston, MA, USA, 2019; ISBN 1789241308.

4. Van Wyk, B.-E. Culinary Herbs and Spices of the World; University of Chicago Press: Chicago, IL, USA, 2014; ISBN 022609183X.

5. Van Wyk, B.-E. A family-level floristic inventory and analysis of medicinal plants used in Traditional African Medicine. J. Ethnopharmacol. 2020, 249, 112351. [CrossRef] [PubMed]

6. Stankovic, M. (Ed.) Lamiaceae Species: Biology, Ecology and Practical Uses; MDPI: Basel, Switzerland, 2020 ; ISBN 9783039284191.

7. Tolley, K.A.; Da Silva, J.M.; Jansen van Vuuren, B. South African National Biodiversity Assessment 2018 Technical Report Volume 7: Genetic Diversity; SANBI: Pretoria, South Africa, 2019.

8. Mamadalieva, N.Z.; Akramov, D.K.; Wessjohann, L.A.; Hussain, H.; Long, C.; Tojibaev, K.S.; Alshammari, E.; Ashour, M.L.; Wink, M. The Genus Lagochilus (Lamiaceae): A review of its diversity, ethnobotany, phytochemistry, and pharmacology. Plants 2021, 10, 132. [CrossRef]

9. Arnold, T.H.; Prentice, C.A.; Hawker, L.C.; Snyman, E.E.; Tomalin, M.; Crouch, N.R.; Pottas-Bircher, C. Medicinal Magical Plants of Southern AFRICA: An Annotated Checklist; National Botanical Institute: Pretoria, South Africa, 2002; ISBN 9780874216561.

10. Nsuala, B.N.; Enslin, G.; Viljoen, A. "Wild cannabis": A review of the traditional use and phytochemistry of Leonotis leonurus. J. Ethnopharmacol. 2015, 174, 520-539. [CrossRef] [PubMed]

11. Aston Philander, L. An ethnobotany of Western Cape Rasta bush medicine. J. Ethnopharmacol. 2011, 138, 578-594. [CrossRef]

12. Van Wyk, B.-E. The potential of South African plants in the development of new food and beverage products. S. Afr. J. Bot. 2011, 77, 857-868. [CrossRef]

13. Asowata-Ayodele, A.M.; Afolayan, A.J.; Otunola, G.A. Ethnobotanical survey of culinary herbs and spices used in the traditional medicinal system of Nkonkobe Municipality, Eastern Cape, South Africa. S. Afr. J. Bot. 2016, 104, 69-75. [CrossRef]

14. Van Wyk, B.-E.; Gorelik, B. The history and ethnobotany of Cape herbal teas. S. Afr. J. Bot. 2017, 110, 18-38. [CrossRef]

15. Van Wyk, B.-E.; Van Oudtshoorn, B.; Gericke, N. Medicinal Plants of South Africa, 2nd ed.; Smit, J., Ed.; Briza Publications: Pretoria, South Africa, 2009; ISBN 978-1-8-75093-37-3.

16. Harley, R.M.; Atkins, S.; Budantsev, A.L.; Cantino, P.D.; Conn, B.J.; Grayer, R.; Harley, M.M.; de Kok, R.; Krestovskaja, T.; Morales, R.; et al. Labiatae BT_Flowering Plants Dicotyledons: Lamiales (except Acanthaceae including Avicenniaceae); Kadereit, J.W., Ed.; Springer: Berlin/Heidelberg, Germany, 2004; pp. 167-275, ISBN 978-3-642-18617-2.

17. Codd, L.E. Flora of Southern Africa: Part 4 Lamiaceae; Leistner, O.A., Ed.; Botanical Research Institute, Departmet of Agriculture and Water Supply, Republic of South Africa: Pretoria, South Africa, 1985; ISBN 9780621082685.

18. Paton, A.J.; Mwanyambo, M.; Govaerts, R.H.A.; Smitha, K.; Suddee, S.; Phillipson, P.B.; Wilson, T.C.; Forster, P.I.; Culham, A. Nomenclatural changes in Coleus and Plectranthus (Lamiaceae): A tale of more than two genera. PhytoKeys 2019, 129, 1-158. [CrossRef]

19. Hiltunen, R.; Holm, Y. Basil: The Genus Ocimum; Harwood Academic Publishers: Amsterdam, The Netherlands, 1999; ISBN 9057024322.

20. Otieno, D.F.; Balkwill, K.; Paton, A.J.; Savolainen, V. A reassessment of Hemizygia and Syncolostemon (Ocimeae-Lamiaceae). Taxon 2006, 55, 941-958. [CrossRef]

21. Marx, H.E.; O’Leary, N.; Yuan, Y.W.; Lu-Irving, P.; Tank, D.C.; Múlgura, M.E.; Olmstead, R.G. A molecular phylogeny and classification of Verbenaceae. Am. J. Bot. 2010, 97, 1647-1663. [CrossRef]

22. Li, B.; Olmstead, R.G.; Zahra, N.; Shinwari, Z.; Kubitzki, K.; Huelsenbeck, J.P.; Ronquist, F.; Boachon, B.; Buell, C.R.; Crisovan, E.; et al. A large-scale chloroplast phylogeny of the Lamiaceae sheds new light on its subfamilial classification. Mol. Phylogenet. Evol. 2016, 6, 1-18. [CrossRef] [PubMed]

23. Frezza, C.; Venditti, A.; Serafini, M.; Bianco, A. Phytochemistry, Chemotaxonomy, Ethnopharmacology, and Nutraceutics of Lamiaceae, 1st ed.; Elsevier B.V.: Amsterdam, The Netherlands, 2019; Volume 62, ISBN 9780444641854.

24. Vladimir-Knezevic, S.; Blazekovic, B.; Kindl, M.; Vladic, J.; Lower-Nedza, A.D.; Brantner, A.H. Acetylcholinesterase inhibitory, antioxidant and phytochemical properties of selected medicinal plants of the Lamiaceae family. Molecules 2014, 19, 767-782. [CrossRef] [PubMed]

25. Štefan, M.B.; Vuković Rodríguez, J.; Blažeković, B.; Kindl, M.; Vladimir-Knežević, S. Total Hydroxycinnamic Acids Assay: Prevalidation and Application on Lamiaceae Species. Food Anal. Methods 2014, 7, 326-336. [CrossRef]

26. Dhifi, W.; Litaiem, M.; Jelali, N.; Hamdi, N.; Mnif, W. Identification of a new chemotye of the plant Mentha aquatica grown in Tunisia: Chemical composition, antioxidant and biological activities of its essential oil. J. Essent. Oil-Bear. Plants 2011, 14, 320-328. [CrossRef] 
27. Esmaeili, A.; Rustaiyan, A.; Masoudi, S.; Nadji, K. Composition of the essential oils of Mentha aquatica L. and Nepeta meyeri Benth. from Iran. J. Essent. Oil Res. 2006, 18, 263-265. [CrossRef]

28. Guetat, A.; Al-Ghamdi, F.A.; Osman, A.K. 1,8-Cineole, $\alpha$-Pinene and Verbenone chemotype of essential oil of species Rosmarinus officinalis L. from Saudi Arabia. Int. J. Herb. Med. 2014, 2, 137-141.

29. Rivett, D.E.A. Isolation of marrubiin from Leonotis leonurus. J. Chem. Soc. 1964, 1857-1858.

30. Dellar, J.E.; Cole-Davies, M.; Waterman, P.G. Unusual antimicrobial compounds from Aeollanthus buchnerianus. Experientia 1996, 52, 175-179. [CrossRef]

31. Chen, Y.S.; Yu, H.M.; Shie, J.J.; Cheng, T.J.R.; Wu, C.Y.; Fang, J.M.; Wong, C.H. Chemical constituents of Plectranthus amboinicus and the synthetic analogs possessing anti-inflammatory activity. Bioorg. Med. Chem. 2014, 22, 1766-1772. [CrossRef]

32. Teixeira, A.P.; Batista, O.; Fátima Simões, M.; Nascimento, J.; Duarte, A.; De La Torre, M.C.; Rodríguez, B. Abietane diterpenoids from Plectranthus grandidentatus. Phytochemistry 1997, 44, 325-327. [CrossRef]

33. Hanson, J.R.; Rivett, D.E.A.; Ley, S.V.; Williams, D.J. The X-ray structure and absolute configuration of insect antifeedant clerodane diterpenoids from Teucrium africanum. J. Chem. Soc. Perkin Trans. 1 1982, 1005-1008. [CrossRef]

34. Iyambo, N.K.; Kibuule, D.; Ilonga, K.S. Antipseudomonal potential of Colophospermum mopane and Acrotome inflata, medicinal plants indigenous to Namibia. Afr. J. Pharm. Pharmacol. 2017, 11, 78-86. [CrossRef]

35. Davies-Coleman, M.T.; Rivett, D.E.A. Structure of the 5,6-dihydro- $\alpha$-pyrone, umuravumbolide. Phytochemistry 1995, 38, 791-792. [CrossRef]

36. Van Puyvelde, L.; De Kimpe, N. Tetradenolide, an $\alpha$-pyrone from Tetradenia riparia. Phytochemistry 1998, 49, 1157-1158. [CrossRef]

37. Collett, L.A.; Davies-Coleman, M.T.; Rivett, D.E.A.; Drewes, S.E.; Horn, M.M. Absolute configuration of $\alpha$-pyrones from Cryptocarya latifolia and Syncolostemon densiflorus. Phytochemistry 1997, 44, 935-938. [CrossRef]

38. Ladan, Z.; Amupitan, J.O.; Oyewale, O.A.; Ayo, R.G.; Temple, E.; Ladan, E.O. Phytochemical screening of the leaf extracts of Hyptis spicigera plant. Afr. J. Pure Appl. Chem. 2014, 8, 83-88. [CrossRef]

39. Matias, D.; Nicolai, M.; Fernandes, A.S.; Saraiva, N.; Almeida, J.; Saraiva, L.; Faustino, C.; Díaz-Lanza, A.M.; Reis, C.P.; Rijo, P. Comparison study of different extracts of Plectranthus madagascariensis, P. neochilus and the rare P. porcatus (Lamiaceae): Chemical characterization, antioxidant, antimicrobial and cytotoxic activities. Biomolecules 2019, 9, 179. [CrossRef]

40. Bianco, A.; Passacantilli, P.; Righi, G.; Nicoletti, M.; Serafini, M. 10-Deoxymelittoside, an iridoid diglucoside, and other iridoids from Lamiastrum galeobdolon. Phytochemistry 1986, 25, 1981-1983. [CrossRef]

41. Nafuka, S.N. In Vitro Antiplasmodial Activity and Phytochemicals Screening of Ethnomedicinal Plants Used to Treat Malaria Associated Symptoms. Ph.D. Thesis, University of Namibia, Windhoek, Namibia, 2014.

42. Grayer, R.J.; Eckert, M.R.; Veitch, N.C.; Kite, G.C.; Marin, P.D.; Kokubun, T.; Simmonds, M.S.J.; Paton, A.J. The chemotaxonomic significance of two bioactive caffeic acid esters, Nepetoidins A and B, in the Lamiaceae. Phytochemistry 2003, 64, 519-528. [CrossRef]

43. Chisowa, E.H.; Mumba, P.; Hall, D.R.; Farman, D.I. Composition of the essential oil of Aeollanthus parvifolius Benth. J. Essent. Oil Res. 1998. [CrossRef]

44. Cui, H.X.; Qiu, Y.; Ge, W.C.; Cheng, F.R.; Yuan, K. Biological activity and phytochemical composition of the volatile oils from Basilicum polystachyon. J. Chem. Soc. Pak. 2017, 39, 43-49.

45. Tan, Y.P.; Xue, Y.; Savchenko, A.I.; Houston, S.D.; Modhiran, N.; McMillan, C.L.D.; Boyle, G.M.; Bernhardt, P.V.; Young, P.R.; Watterson, D.; et al. Basimarols A, B, and C, highly oxygenated Pimarane Diterpenoids from Basilicum polystachyon. J. Nat. Prod. 2019, 82, 2828-2834. [CrossRef] [PubMed]

46. Tan, Y.P.; Houston, S.D.; Modhiran, N.; Savchenko, A.I.; Boyle, G.M.; Young, P.R.; Watterson, D.; Williams, C.M. Stachyonic Acid: A Dengue Virus inhibitor from Basilicum polystachyon. Chem. A Eur. J. 2019, 25, 5664-5667. [CrossRef] [PubMed]

47. Touani, F.K.; Seukep, A.J.; Djeussi, D.E.; Fankam, A.G.; Noumedem, J.A.K.; Kuete, V. Antibiotic-potentiation activities of four Cameroonian dietary plants against multidrug-resistant Gram-negative bacteria expressing efflux pumps. BMC Complement. Altern. Med. 2014, 14, 258. [CrossRef]

48. Koba, K.; Raynaud, C.; Millet, J.; Chaumont, J.-P.; Sanda, K. Chemical composition of Hyptis pectinata L., H. lanceolata Poit, H. suaveolens (L) Poit and H. spicigera Lam. essential oils from Togo. J. Essent. Oil-Bear. Plants 2007, 10, 357-364. [CrossRef]

49. Aycard, J.-P.; Kini, F.; Kam, B.; Gaydou, E.M.; Faure, R. Isolation and Identification of Spicigera Lactone: Complete 1H and 13C assignments using Two-Dimensional NMR Experiments. J. Nat. Prod. 1993, 56, 1171-1173. [CrossRef]

50. Kini, F.; Kam, B.; Aycard, J.P.; Gaydou, E.M.; Bombarda, I. Chemical composition of the essential oil of Hyptis spicigera Lam. from Burkina Faso. J. Essent. Oil Res. 1993, 5, 219-221. [CrossRef]

51. Fragoso-Serrano, M.; González-Chimeo, E.; Pereda-Miranda, R. Novel labdane diterpenes from the insecticidal plant Hyptis spicigera. J. Nat. Prod. 1999, 62, 45-50. [CrossRef]

52. Bailac, P.; Duschatzky, C.; Ponzi, M.; Firpo, N. Essential oil of Hyptis mutabilis (Rich.) Briq. Grown in San Luis, Argentina. J. Essent. Oil Res. 1999, 11, 217-219. [CrossRef]

53. Pereda-Miranda, R.; Gascon-Figueroa, M. Chemistry of Hyptis mutabilis: New pentacyclic triterpenoids. J. Nat. Prod. 1988, 51, 996. [CrossRef]

54. McNeil, M.; Facey, P.; Porter, R. Essential oils from the Hyptis genus-A review (1909-2009). Nat. Prod. Commun. 2011, 6, 1775-1796. [CrossRef] 
55. Velasco-Negueruela, A.; Perez-Alonso, M.J.; Esteban, J.L.; Guzman, C.A.; Zygadlo, J.A.; Espinar, L.A. Volatile constituents of Hyptis mutabilis (Rich.) Briq. J. Essent. Oil Res. 1995, 7, 81. [CrossRef]

56. López-Garcia, R.E.; Hernandez-Perez, M.; Rabanal, R.M.; Darias, V.; Martin-Herrera, D.; Arias, A.; Sanz, J. Essential oils and antimicrobial activity of two varieties of Cedronella canariensis (L.) W. et B. J. Ethnopharmacol. 1992, 36, 207. [CrossRef]

57. Carreiras, M.E.; Rodríguez, B.; López-Garcia, R.E.; Rabanal, R.M. A dimer of D-Pinocarvone from Cedronella canariensis. Phytochemistry 1987, 26, 3351-3353. [CrossRef]

58. Abdel-Mogib, M.; Albar, H.A.; Batterjee, S.M. Chemistry of the genus Plectranthus. Molecules 2002, 7, 271-301. [CrossRef]

59. Arumugam, G.; Sinniah, U.R.; Swamy, M.K.; Lynch, P.T. Micropropagation and essential oil characterization of Plectranthus amboinicus (Lour.) Sprengel, an aromatic medicinal plant. In Vitro Cell. Dev. Biol. Plant 2020, 56, 491-503. [CrossRef]

60. Janakiraman, D.; Parameswari, C.S. Active phenolic constituents and brine shrimp lethality assay of hydroalcoholic extract of Plectranthus amboinicus. Asian J. Pharm. Clin. Res. 2017, 10, 186-189. [CrossRef]

61. Ashaari, N.S.; Rahim, M.H.A.; Sabri, S.; Lai, K.S.; Song, A.A.L.; Rahim, R.A.; Wan Abdullah, W.M.A.N.; Abdullah, J.O. Functional characterization of a new terpene synthase from Plectranthus amboinicus. PLoS ONE 2020, 15, e0235416. [CrossRef]

62. Aguiar, J.J.S.; Sousa, C.P.B.; Araruna, M.K.A.; Silva, M.K.N.; Portelo, A.C.; Lopes, J.C.; Carvalho, V.R.A.; Figueredo, F.G.; Bitu, V.C.N.; Coutinho, H.D.M.; et al. Antibacterial and modifying-antibiotic activities of the essential oils of Ocimum gratissimum L. and Plectranthus amboinicus L. Eur. J. Integr. Med. 2015, 7, 151-156. [CrossRef]

63. Wibisono, K.; Aisyah, S.I.; Suhesti, S.; Nurcholis, W.; Science, N.; Biopharmaca, T. Optimization of total flavonoids extraction and A-glucosidase inhibitory activity from Plectranthus amboinicus (Lour.) Spreng. leaves using the simplex-centroid design. Molekul 2019, 14, 84-91. [CrossRef]

64. Bezerra, R.d.C.d.F.; Neto, F.B.d.O.; da Silva, F.F.M.; Bertini, L.M.; Alves, L.A. Seasonal effect in essential oil composition and antioxidant activity of Plectranthus amboinicus leaves. Biosci. J. 2017, 1608-1616. [CrossRef]

65. Nguyen, N.Q.; Minh, L.V.; Trieu, L.H.; Bui, L.M.; Lam, T.D.; Hieu, V.Q.; Khang, T.V.; Trung, L.N.Y. Evaluation of total polyphenol content, total flavonoid content, and antioxidant activity of Plectranthus amboinicus leaves. IOP Conf. Ser. Mater. Sci. Eng. 2020, 736. [CrossRef]

66. Bugayong, A.M.; Cruz, P.; Padilla, P.I. Antibacterial activity and chemical composition of essential oils from leaves of some aromatic plants of Philippines. J. Essent. Oil Bear. Plants 2019, 22, 932-946. [CrossRef]

67. Hsu, K.P.; Ho, C.L. Antimildew effects of Plectranthus amboinicus leaf essential oil on paper. Nat. Prod. Commun. 2019, 14. [CrossRef]

68. Murthy, P.S.; Ramalakshmi, K.; Srinivas, P. Fungitoxic activity of Indian borage (Plectranthus amboinicus) volatiles. Food Chem. 2009, 114, 1014-1018. [CrossRef]

69. Gelmini, F.; Squillace, P.; Testa, C.; Sparacino, A.C.; Angioletti, S.; Beretta, G. GC-MS characterisation and biological activity of essential oils from different vegetative organs of Plectranthus barbatus and Plectranthus caninus cultivated in north Italy. Nat. Prod. Res. 2015, 29, 993-998. [CrossRef]

70. Alasbahi, R.H.; Melzig, M.F. Plectranthus barbatus: A review of phytochemistry, ethnobotanical uses and pharmacology-Part 1. Planta Med. 2010, 76, 653-661. [CrossRef]

71. Falé, P.L.; Borges, C.; Madeira, P.J.A.; Ascensão, L.; Araújo, M.E.M.; Florêncio, M.H.; Serralheiro, M.L.M. Rosmarinic acid, scutellarein $4^{\prime}$-methyl ether 7-O-glucuronide and (16S)-coleon $\mathrm{E}$ are the main compounds responsible for the antiacetylcholinesterase and antioxidant activity in herbal tea of Plectranthus barbatus ("falso boldo"). Food Chem. 2009, 114, 798-805. [CrossRef]

72. Kelecom, A.; Dos Santos, T.C. Cariocal, a new Seco-Abietane Diterpene from the Labiate Coleus barbatus. Tetrahedron Lett. 1985, 26, 3659-3662. [CrossRef]

73. Kelecom, A.; Dos Santos, T.C.; Medeiros, W.L.B. Secoabitane Diterpenes from Coleus barbatus. Phytochemistry 1987, 26, 2337-2340. [CrossRef]

74. Kelecom, A. Isolation, structure determination, and absolute configuration of barbatusol, a new bioactive diterpene with a rearranged abietane skeleton from the Labiate Coleus barbatus. Tetrahedron 1983, 39, 3603-3608. [CrossRef]

75. Kelecom, A. An abietane Diterpene from the Labiate Coleus barbatus. Phytochemistry 1984, 23, 1677-1679. [CrossRef]

76. Musayeib, N.M.; Al Amina, M.; Al-hamoud, G.A.; Mohamed, G.A.; Ibrahim, S.R.M.; Shabana, S. Plectrabarbene, a new abietane diterpene from Plectranthus barbatus aerial parts. Molecules 2020, 25, 2365. [CrossRef]

77. Schultz, C.; Bossolani, M.P.; Torres, L.M.B.; Lima-Landman, M.T.R.; Lapa, A.J.; Souccar, C. Inhibition of the gastric H+,K+-ATPase by plectrinone A, a diterpenoid isolated from Plectranthus barbatus Andrews. J. Ethnopharmacol. 2007, 111, 1-7. [CrossRef]

78. Cuthbertson, D.J.; Johnson, S.R.; Piljac-Žegarac, J.; Kappel, J.; Schäfer, S.; Wüst, M.; Ketchum, R.E.B.; Croteau, R.B.; Marques, J.V.; Davin, L.B.; et al. Accurate mass-time tag library for LC/MS-based metabolite profiling of medicinal plants. Phytochemistry 2013, 91, 187-197. [CrossRef]

79. Banthorpe, D.V.; Bilyard, H.J.; Brown, G.D. Enol esters of caffeic acid in several genera of the Labiatae. Phytochemistry 1989, 28, 2109-2113. [CrossRef]

80. Mota, L.; Figueiredo, A.C.; Pedro, L.G.; Barroso, J.G.; Miguel, G.M.; Faleiro, M.L.; Ascensão, L. Volatile-oils composition, and bioactivity of the essential oils of Plectranthus barbatus, P. neochilus, and P. ornatus grown in Portugal. Chem. Biodivers. 2014, 11, 719-732. [CrossRef] 
81. Mesquita, L.S.F.; Matos, T.S.; Do Nascimento Ávila, F.; Da Silva Batista, A.; Moura, A.F.; De Moraes, M.O.; Da Silva, M.C.M.; Ferreira, T.L.A.; Nascimento, N.R.F.; Monteiro, N.K.V.; et al. Diterpenoids from leaves of cultivated Plectranthus ornatus. Planta Med. 2020. [CrossRef]

82. Passinho-Soares, H.C.; Meira, P.R.; David, J.P.; Mesquita, P.R.R.; Do Vale, A.E.; De Rodrigues, F.M.; De Pereira, P.A.P.; De Santana, J.R.F.; De Oliveira, F.S.; De Andrade, J.B.; et al. Volatile organic compounds obtained by in vitro callus cultivation of Plectranthus ornatus Codd. (Lamiaceae). Molecules 2013, 18, 10320-10333. [CrossRef]

83. Rijo, P.; Rodriguez, B.; Duarte, A.; Fatima Simoes, M. Antimicrobial Properties of Plectranthus ornatus Extracts, 11-acetoxyhalima-5, 13-dien-15-oic Acid Metabolite and its Derivatives. Nat. Prod. J. 2011, 1, 57-64. [CrossRef]

84. Rijo, P.; Gaspar-Marques, C.; Simões, M.F.; Duarte, A.; del Apreda-Rojas, M.C.; Cano, F.H.; Rodríguez, B. Neoclerodane and labdane diterpenoids from Plectranthus ornatus. J. Nat. Prod. 2002, 65, 1387-1390. [CrossRef] [PubMed]

85. Medrado, H.H.; Dos Santos, E.O.; Ribeiro, E.M.O.; David, J.M.; David, J.P.; Araújo, J.F.; Do Vale, A.E.; Bellintani, M.C.; Brandão, H.N.; Meira, P.R. Rosmarinic and cinnamic acid derivatives of in vitro tissue culture of Plectranthus ornatus: Overproduction and correlation with antioxidant activities. J. Braz. Chem. Soc. 2017, 28, 505-511. [CrossRef]

86. Ávila, F.N.; Pinto, F.C.L.; Sousa, T.S.; Torres, M.C.M.; Costa-Lotufo, L.V.; Rocha, D.D.; De Vasconcelos, M.A.; Cardoso-Sá, N.; Teixeira, E.H.; Albuquerque, M.R.J.R.; et al. Miscellaneous diterpenes from the aerial parts of Plectranthus ornatus Codd. J. Braz. Chem. Soc. 2017, 28, 1014-1022. [CrossRef]

87. Rijo, P.; Gaspar-Marques, C.; Simões, M.F.; Jimeno, M.L.; Rodríguez, B. Further diterpenoids from Plectranthus ornatus and P. grandidentatus. Biochem. Syst. Ecol. 2007, 35, 215-221. [CrossRef]

88. Otaifah, Y.N.; Bouyahya, A.; Talbaoui, A.; Harhar, H.; El Hajjaji, S. Chemical composition of Yemeni medicinal plants essentials oils and their antibacterial and antioxidant activities. Phytothérapie 2018. [CrossRef]

89. Amina, M.; Al Musayeib, N.M.; Mohamed, G.A.; Ibrahim, S.R.M. Plectraterpene, a new ursane-type triterpene ester and other steroids from the aerial parts of Plectranthus montanus. Rev. Bras. Farmacogn. 2017, 27, 698-701. [CrossRef]

90. Grayer, R.J.; Eckert, M.R.; Lever, A.; Veitch, N.C.; Kite, G.C.; Paton, A.J. Distribution of exudate flavonoids in the genus Plectranthus. Biochem. Syst. Ecol. 2010, 38, 335-341. [CrossRef]

91. Maistry, K. The Antimicrobial Properties and Chemical Composition of Leaf Extracts and Essential Oils of Indigenous Plectranthus Species. Ph.D. Thesis, University of the Witwatersand, Johannesburg, South Africa, 2007.

92. Uchida, M.; Miyase, T.; Yoshizaki, F.; Bieri, J.H.; Riiedi, P.; Eugster, C.H. 4-Hydroxytaxodion als Hauptditerpen in Plectranthus grandidentatus Gurke. Helv. Chim. Acta 1981, 64, 2227-2250. [CrossRef]

93. Ibrahim, M.E.; Ahmed, S.S.; Hussein, M.S. Chemical investigations and the antimicrobial activity of Ocimum hadiensis (Forssk) plant grown wild in Egypt. J. Mater. Environ. Sci. 2019, 2508, 457-462.

94. Dukhea, S. The Isolation, Structure Elucidation and Biological Testing of Compounds from Plectranthus hadiensis. Ph.D. Thesis, University of KwaZulu-Natal, Durban, South Africa, 2010.

95. Sripathi, R.; Ravi, S. Chemical composition and antibacterial activity of the essential oil from the seeds of Plectranthus hadiensis. Int. J. Pharmacogn. Phytochem. Res. 2017, 9, 637-639. [CrossRef]

96. Gurlal, P. Testing for Microbiologically Active Compounds Extracted from Members of the Family Lamiaceae and Other Indigenous Plants. Ph.D. Thesis, University of KwaZulu-Natal, Pietermaritzburg, South Africa, 2005.

97. Van Zyl, R.L.; Khan, F.; Edwards, T.J.; Drewes, S.E. Antiplasmodial activities of some abietane diterpenes from the leaves of five Plectranthus species. S. Afr. J. Sci. 2008, 104, 62-64.

98. Batista, O.; Duarte, A.; Nascimento, J.; Simōes, M.F.; de la Torre, M.C.; Rodríguez, B. Structure and antimicrobial activity of diterpenes from the roots of Plectranthus hereroensis. J. Nat. Prod. 1994, 57, 858-861. [CrossRef]

99. Batista, O.; Fátima Simões, M.; Duarte, A.; Luisa Valdeira, M.; de la Torre, M.C.; Rodríguez, B. An antimicrobial abietane from the root of Plectranthus hereroensis. Phytochemistry 1995, 38, 167-169. [CrossRef]

100. Rodriguez, B.; Maria, C.; Simoes, F.; Batsta, O.; Nascimento, J.; Duartet, A.; Mayer, R. Revision of the structure of an Aristolane Sesquiterpene Aldehyde isolated from the root of Plectranthus hereroensis and Aristolochia debilis. Phytochemistry 1995, 38, 905-907. [CrossRef]

101. Ascensao, L.; Figueiredo, A.C.; Barroso, J.G.; Pedro, L.G.; Schripsema, J.; Deans, S.G.; Scheffer, J.J.C. Plectranthus madagascariensis: Morphology of the glandular trichomes, essential oil composition, and its biological activity. Int. J. Plant Sci. 1998, 159, 31-38. [CrossRef]

102. Kubinova, R.; Porizkova, R.; Navratilova, A.; Farsa, O.; Hanakova, Z.; Bacinska, A.; Cizek, A.; Valentova, M. Antimicrobial and enzyme inhibitory activities of the constituents of Plectranthus madagascariensis (Pers.) Benth. J. Enzyme Inhib. Med. Chem. 2014, 29, 749-752. [CrossRef]

103. Matias, D.; Nicolai, M.; Saraiva, L.; Pinheiro, R.; Faustino, C.; Diaz Lanza, A.; Pinto Reis, C.; Stankovic, T.; Dinic, J.; Pesic, M.; et al. Cytotoxic activity of royleanone diterpenes from Plectranthus madagascariensis Benth. ACS Omega 2019, 4, 8094-8103. [CrossRef]

104. Aguiar, G.P.; Lima, K.A.; Severiano, M.E.; Groppo, M.; Ambrosio, S.R.; Crevelin, E.J. Antifungal activity of the essential oils of Plectranthus neochilus (Lamiaceae) and Tagetes erecta (Asteraceae) cultivated in Brazil. Int. J. Complement. Altern. Med. 2018, 11, 1-5. [CrossRef]

105. El-Sakhawy, F.S.; Kassem, H.A.; El-Gayed, S.H.; Mostafa, M.M. Headspace Solid Phase Microextraction Analysis of volatile compounds of the aerial parts and flowers of Plectranthus neochilus Schltr. and Salvia farinacea Benth. J. Essent. Oil-Bear. Plants 2018, 21, 674-686. [CrossRef] 
106. Lambrechts, I.A.; Lall, N. Plectranthus neochilus. In Underexplored Medicinal Plants from Sub-Saharan Africa; Lall, N., Ed.; Academic Press: London, UK, 2020; pp. 235-240, ISBN 9780128168141.

107. Fanela, T.L.M.; Baldin, E.L.L.; Pannuti, L.E.R.; Cruz, P.L.; Crotti, A.E.M.; Takeara, R.; Kato, M.J. Lethal and inhibitory activities of plant-derived essential oils against Bemisia tabaci Gennadius (Hemiptera: Aleyrodidae) Biotype B in tomato. Neotrop. Entomol. 2016, 45, 201-210. [CrossRef]

108. Da Cruz Marçalo de Andrade, J.E. Unravelling New Ethnopharmacological Roles of Plectranthus Species: Biological Activity Screening. Ph.D. Thesis, University of Lisbon, Lisbon, Portugal, 2016.

109. Rijo, P. Phytochemical Study and Biological Activities of Diterpenes and Derivatives from Plectranthus Species. Ph.D. Thesis, University of Lisbon, Lisbon, Portugal, 2010.

110. Manikandan, S.; Alagu Lakshmanan, G.; Chandran, C.; Alagu Lakshmanan, C.G. Phytochemical screening and evaluation of tuber extract of Plectranthus rotundifolius Spreng. by GC-MS and FT-IR spectrum analysis. Int. J. Herb. Med. 2016, 36, 36-40.

111. Maree, J.E.; Khondkar, P.; Kwapong, A.A.; Oyedemi, B.M.; Aljarba, T.M.; Stapleton, P.; Viljoen, A.M.; Gibbons, S. Bioactive acetophenones from Plectranthus venteri. Phytochem. Lett. 2014, 10. [CrossRef]

112. Chagonda, L.S.; Chalchat, J.-C. The essential oil of wild and cultivated Hoslundia opposita Vahl. from Zimbabwe. Flavour Fragr. J. 2005, 20, 193-195. [CrossRef]

113. Achenbach, H.; Waibel, R.; Nkunya, M.H.H.; Weenen, H. Antimalarial compounds from Hoslundia opposita. Phytochemistry 1992, 31, 3781-3784. [CrossRef]

114. Ngadjui, B.T.; Ayafor, J.F.; Sondengam, B.L.; Connolly, J.D.; Rycroft, D.S. Hoslundin, hoslundal, and hoslunddiol: Three new flavonoids from the twigs of Hoslundia opposita (Lamiaceae). Tetrahedron 1991, 47, 3555. [CrossRef]

115. Usman, L.A.; Zubair, M.F.; Adebayo, S.A.; Oladosu, I.A.; Muhammad, N.O.; Akolade, J.O. Chemical composition of leaf and fruit essential oils of Hoslundia opposita Vahl grown in Nigeria. Am. J. Agric. Environ. Sci. 2010, 8, 40-43.

116. Ngadjui, B.T.; Ayafor, J.F.; Sondengam, B.L.; Connolly, J.D.; Rycroft, D.S.; Tillequin, F. Oppositin and 5-O-Methylhoslundin, pyrone-substituted flavonoids of Hoslundia opposita. Phytochemistry 1993, 32, 1313-1315. [CrossRef]

117. Ngadjui, B.T.; Tsopmo, A.; Ayafor, J.F.; Connolly, J.D.; Tamboue, H. Hosloppin, a new pyrone-substituted flavonoid from Hoslundia opposita. J. Nat. Prod. 1995, 58, 109. [CrossRef]

118. Sajjadi, S.E.; Ghannadi, A. Analysis of the essential oil of Lamium amplexicaule L. from Northeastern Iran. J. Essent. Oil Bear. Plants 2012, 15, 577-581. [CrossRef]

119. Yalçin, F.N.; Kaya, D. Ethnobotany, pharmacology and phytochemistry of the genus Lamium (Lamiaceae). Fabad J. Pharm. Sci. 2006, 31, 43-53.

120. Nickavar, B.; Mojab, F.; Bamasian, S. Volatile components from aerial parts of Lamium amplexicaule from Iran. J. Essent. Oil Bear. Plants 2008, 11, 36-40. [CrossRef]

121. Alipieva, K.; Kokubun, T.; Taskova, R.; Evstatieva, L.; Handjieva, N. LC-ESI-MS analysis of iridoid glucosides in Lamium species. Biochem. Syst. Ecol. 2007, 35, 17-22. [CrossRef]

122. Alipieva, K.I.; Taskova, R.M.; Evstatieva, L.N.; Handjieva, N.V.; Popov, S.S. Benzoxazinoids and iridoid glucosides from four Lamium species. Phytochemistry 2003, 64, 1413-1417. [CrossRef] [PubMed]

123. El-Hela, A.A.; Abdel-Hady, N.M.; Dawoud, G.T.; Ghoneim, M.M. HPTLC fingerprint profile of triterpenes of Lamium amplexicaule Benth. and Ajuga iva L. (Lamiaceae) monitored with screening of their anti-inflammatory effect. J. Pharmacogn. Phytochem. 2016, 5, 176-181.

124. Guiso, M.; Martino, C. 6-Deoxylamioside, a new iridoid glucoside from Lamium amplexicaule. J. Nat. Prod. 1983, 46, 157-160. [CrossRef]

125. Kikuchi, M.; Onoguchi, R.; Yaoita, Y. Three new monoterpene glucosides from Lamium amplexicaule. Helv. Chim. Acta 2009, 92, 2063-2070. [CrossRef]

126. Alipieva, K.I.; Evstatieva, L.; Handjieva, N.; Popov, S. Comparative analysis of the composition of flower volatiles from Lamium L. species and Lamiastrum galeobdolon Heist. ex Fabr. Z. Naturforsch. Sect. C J. Biosci. 2003, 58, 779-782. [CrossRef]

127. Nsuala, B.N.; Kamatou, G.P.P.; Sandasi, M.; Enslin, G.; Viljoen, A. Variation in essential oil composition of Leonotis leonurus, an important medicinal plant in South Africa. Biochem. Syst. Ecol. 2017, 70, 155-161. [CrossRef]

128. He, F.; Lindqvist, C.; Harding, W.W. Leonurenones A-C: Labdane diterpenes from Leonotis leonurus. Phytochemistry 2012, 83, 168-172. [CrossRef]

129. Oyedeji, O.A.; Afolayan, A.J. Comparative study of the essential oil composition and antimicrobial activity of Leonotis leonurus and L. ocymifolia in the Eastern Cape, South Africa. S. Afr. J. Bot. 2005, 71, 114-116. [CrossRef]

130. Naidoo, D.; Maharaj, V.; Crouch, N.R.; Ngwane, A. New labdane-type diterpenoids from Leonotis leonurus support circumscription of Lamiaceae s.l. Biochem. Syst. Ecol. 2011, 39, 216-219. [CrossRef]

131. Vallabh, J.B. The Antimicrobial Properties and Chemical Composition of Leaf Essential Oils of Selected Lamiaceae Species in South Africa. Ph.D. Thesis, University of the Witwatersrand, Johannesburg, South Africa, 2003.

132. Wu, H.; Li, J.; Fronczek, F.R.; Ferreira, D.; Burandt, C.L.; Setola, V.; Roth, B.L.; Zjawiony, J.K. Labdane diterpenoids from Leonotis leonurus. Phytochemistry 2013, 91, 229-235. [CrossRef]

133. Pedro, L.G.; Barroso, J.G.; Marques, N.T.; Ascensao, L.; Pais, M.S.S.; Scheffer, J.J.C. Composition of the essential oil from sepals of Leonotis leonurus R. Br. J. Essent. Oil Res. 1991, 3, 451. [CrossRef] 
134. Vos, W.T. A Systematic Study of Leonotis (Pers.) R.Br. (Lamiaceae) in Southern Africa. Ph.D. Thesis, University of Natal, Pietermaritzburg, South Africa, 1995.

135. Agnihotri, V.K.; ElSohly, H.N.; Smillie, T.J.; Khan, I.A.; Walker, L.A. Constituents of Leonotis leonurus flowering tops. Phytochem. Lett. 2009, 2, 103-105. [CrossRef]

136. Cragg, G.M.L.; Little, G.E. The extractives of Leonotis leonurus. J. S. Afr. Chem. Inst. 1962, 15, 29-30.

137. El-Ansari, M.A.; Aboutabl, E.A.; Farrag, A.R.H.; Sharaf, M.; Hawas, U.W.; Soliman, G.M.; El-Seed, G.S. Phytochemical and pharmacological studies on Leonotis leonurus. Pharm. Biol. 2009, 47, 894-902. [CrossRef]

138. Kaplan, E.R.; Rivett, D.E.A. Structures of compounds X and Y, two labdane diterpenoids, from Leonotis leonurus. J. Chem. Soc. C Org. 1968, 262. [CrossRef]

139. Kuchta, K.; Volk, R.B.; Rauwald, H.W. Stachydrine in Leonurus cardiaca, Leonurus japonicus, Leonotis leonurus: Detection and quantification by instrumental HPTLC and 1H-qNMR analyses. Pharmazie 2013, 68, 534-540. [CrossRef]

140. Mazimba, O. Leonotis leonurus: A herbal medicine review. J. Pharmacogn. Phytochem. 2015, 3, 74-82.

141. McKenzie, J.M.; Green, I.R.; Mugabo, P. Leonurun, a novel labdane diterpenoid from Leonotis leonurus. S. Afr. J. Chem. 2006, 59, 114-116.

142. Naidu, K. An Investigation of Compounds Occuring in Leonotis Species. Ph.D. Thesis, Rhodes University, Grahamstown, South Africa, 1970.

143. Narukawa, Y.; Komori, M.; Niimura, A.; Noguchi, H.; Kiuchi, F. Two new diterpenoids from Leonotis leonurus R. Br. J. Nat. Med. 2015, 69, 130-134. [CrossRef]

144. Tonisi, S.; Okaiyeto, K.; Hoppe, H.; Mabinya, L.V.; Nwodo, U.U.; Okoh, A.I. Chemical constituents, antioxidant and cytotoxicity properties of Leonotis leonurus used in the folklore management of neurological disorders in the Eastern Cape, South Africa. 3 Biotech 2020, 10, 1-14. [CrossRef]

145. Damasceno, L.M.O.; Silva, A.L.N.; dos Santos, R.F.; Feitosa, T.A.; Viana, L.G.F.; de Oliveira, R.G.; e Silva, M.G.; Rolim, L.A.; Araújo, C.S.; Araújo, E.C.C.; et al. Cytotoxic activity of chemical constituents and essential oil from the leaves of Leonotis nepetifolia (Lamiaceae). Rev. Virtual Quim. 2019, 11, 517-528. [CrossRef]

146. Li, J.; Fronczek, F.R.; Ferreira, D.; Burandt, C.L.; Setola, V.; Roth, B.L.; Zjawiony, J.K. Bis-spirolabdane diterpenoids from Leonotis nepetaefolia. J. Nat. Prod. 2012, 75, 728-734. [CrossRef]

147. Muhayimana, A.; Chalchat, J.C.; Garry, R.-P. Chemical composition of essential oils of some medicinal plants from Rwanda. J. Essent. Oil Res. 1998, 10, 251-259. [CrossRef]

148. Oliveira, D.M.; Melo, F.G.; Balogun, S.O.; Flach, A.; De Souza, E.C.A.; De Souza, G.P.; Rocha, I.D.N.A.; Da Costa, L.A.M.A.; Soares, I.M.; Da Silva, L.I.; et al. Antibacterial mode of action of the hydroethanolic extract of Leonotis nepetifolia (L.) R. Br. involves bacterial membrane perturbations. J. Ethnopharmacol. 2015, 172, 356-363. [CrossRef]

149. Ueda, F.; Iizuka, K.; Tago, K.; Narukawa, Y.; Kiuchi, F.; Kasahara, T.; Tamura, H.; Funakoshi-Tago, M. Nepetaefuran and leonotinin isolated from Leonotis nepetaefolia $\mathrm{R}$. Br. potently inhibit the LPS signaling pathway by suppressing the transactivation of NF- $\mathrm{B}$. Int. Immunopharmacol. 2015, 28, 967-976. [CrossRef]

150. Nibret, E.; Wink, M. Trypanocidal and antileukaemic effects of the essential oils of Hagenia abyssinica, Leonotis ocymifolia, Moringa stenopetala, and their main individual constituents. Phytomedicine 2010, 17, 911-920. [CrossRef] [PubMed]

151. Kruger, G.J.; Rivett, D.E.A. Diterpenoids of Leonotis species. Part 6.1 The crystal and molecular structure of leonitin, a 9,13epoxylabdane. S. Afr. J. Chem. 1979, 32, 61-62. [CrossRef]

152. Vagionas, K.; Graikou, K.; Chinou, I.B.; Runyoro, D.; Ngassapa, O. Chemical analysis and antimicrobial activity of essential oils from the aromatic plants Artemisia afra Jacq. and Leonotis ocymifolia (Burm. F.) Iwarsson var. raineriana (Vision1) Iwarsson growing in Tanzania. J. Essent. Oil Res. 2007, 19, 396-400. [CrossRef]

153. Habtemariam, S.; Gray, A.I.; Waterman, P.G. Diterpenes from the leaves of Leonotis ocymifolia var. raineriana. J. Nat. Prod. 1994, 57, 1570-1574. [CrossRef]

154. Rivett, D.E.A. The structure of lasiocoryin, a Grindelane Diterpenoid from Lasiocorys capensis Benth. (Labiate). S. Afr. J. Chem. 1975, 28, 305-311.

155. Vagionas, K.; Ngassapa, O.; Runyoro, D.; Graikou, K.; Gortzi, O.; Chinou, I. Chemical analysis of edible aromatic plants growing in Tanzania. Food Chem. 2007, 105, 1711-1717. [CrossRef]

156. Eze, U.A.; Bello, S.O.; Etuk, E.U.; Ameh, G.I.; Ugwah, O.M.; Ugwah-oguejiofor, C.J. Phytochemical and preliminary toxicological studies of the aqueous leave extract of Leucas martinicensis in wistar rats. Int. J. Med. Plants Res. 2013, 2, 166-169.

157. Kadri, A.; Zarai, Z.; Ben Chobba, I.; Bekir, A.; Gharsallah, N.; Damak, M.; Gdoura, R. Chemical composition and antioxidant activity of Marrubium vulgare L. essential oil from Tunisia. Afr. J. Biotechnol. 2011, 10, 3908-3914.

158. Ahmed, B.; Masoodi, M.H.; Siddique, A.H.; Khan, S. A new monoterpene acid from Marrubium vulgare with potential antihepatotoxic activity. Nat. Prod. Res. 2010, 24, 1671-1680. [CrossRef]

159. Zarai, Z.; Kadri, A.; Ben Chobba, I.; Ben Mansour, R.; Bekir, A.; Mejdoub, H.; Gharsallah, N. The in vitro evaluation of antibacterial, antifungal and cytotoxic properties of Marrubium vulgare L. essential oil grown in Tunisia. Lipids Health Dis. 2011, 10, 161. [CrossRef]

160. Amessis-Ouchemoukh, N.; Abu-Reidah, I.M.; Quirantes-Piné, R.; Madani, K.; Segura-Carretero, A. Phytochemical profiling, in vitro evaluation of total phenolic contents and antioxidant properties of Marrubium vulgare (horehound) leaves of plants growing in Algeria. Ind. Crops Prod. 2014, 61, 120-129. [CrossRef] 
161. Nagy, M.; Svajdlenka, E. Comparison of essential oils from Marrubium vulgare L. and M. peregrinum L. J. Essent. Oil Res. 1998, 10, 585-587. [CrossRef]

162. Amri, B.; Martino, E.; Vitulo, F.; Corana, F.; Ben-Kaâb, L.B.; Rui, M.; Rossi, D.; Mori, M.; Rossi, S.; Collina, S. Marrubium vulgare L. leave extract: Phytochemical composition, antioxidant and wound healing properties. Molecules 2017, 22, 1851. [CrossRef]

163. Khanavi, M.; Ghasemian, L.; Motlagh, E.H.; Hadjiakhoondi, A.; Shafiee, A. Chemical composition of the essential oils of Marrubium parviflorum Fisch. and C. A. Mey. and Marrubium vulgare L. from Iran. Flavour Fragr. J. 2005, 20, 324-326. [CrossRef]

164. Aćimović, M.; Jeremić, K.; Salaj, N.; Gavarić, N.; Kiprovski, B.; Sikora, V.; Zeremski, T. Marrubium vulgare L.: A phytochemical and pharmacological overview. Molecules 2020, 25, 2898. [CrossRef] [PubMed]

165. Chaker, A.N.; Boukhebti, H.; Sahraoui, R.; Ramdhani, M. Essential oils and morphological study of Mentha aquatica. Pharmacogn. Commun. 2014, 4, 34-38. [CrossRef]

166. Benabdallah, A.; Rahmoune, C.; Boumendjel, M.; Aissi, O.; Messaoud, C. Total phenolic content and antioxidant activity of six wild Mentha species (Lamiaceae) from northeast of Algeria. Asian Pac. J. Trop. Biomed. 2016, 6, 760-766. [CrossRef]

167. Safaiee, P.; Taghipour, A.; Vahdatkhoram, F.; Movagharnejad, K. Extraction of phenolic compounds from Mentha aquatica: The effects of sonication time, temperature and drying method. Chem. Pap. 2019, 73, 3067-3073. [CrossRef]

168. Stagos, D.; Portesis, N.; Spanou, C.; Mossialos, D.; Aligiannis, N.; Chaita, E.; Panagoulis, C.; Reri, E.; Skaltsounis, L.; Tsatsakis, A.M.; et al. Correlation of total polyphenolic content with antioxidant and antibacterial activity of 24 extracts from Greek domestic Lamiaceae species. Food Chem. Toxicol. 2012, 50, 4115-4124. [CrossRef]

169. Senatore, F.; D’Alessio, A.; Formisano, C.; Özcan, M. Chemical composition and antibacterial activity of the essential oil of a 1,8-cineole chemotype of Mentha aquatica L. growing wild in Turkey. J. Essent. Oil Bear. Plants 2005, 8, 148-153. [CrossRef]

170. Ferhat, M.; Kabouche, Z.; Fujimoto, Y.; Araya, H. Two new triterpenes and other compounds from Mentha aquatica (Lamiaceae). Nat. Prod. Commun. 2017, 12, 483-486. [CrossRef]

171. Malingré, T.M.; Maarse, H. The composition of the essential oil of Mentha aquatica. Phytochemistry 1974, 13, 1531-1535. [CrossRef]

172. Mentha aquatica L. In Natural Compound_Flavonoids; Azimova, S.S., Vinogradova, V.I., Eds.; Springer Science+Business Media: New York, NY, USA, 2013; ISBN 9781461405351.

173. Morteza-Semnani, K.; Saeedi, M.; Akbarzadeh, M. The essential oil composition of Mentha aquatica L. J. Essent. Oil Bear. Plants 2006, 9, 283-286. [CrossRef]

174. Burzanska-Hermann, Z.; Rzadkowska-Bodalska, H.; Olechnowicz-Stepien, W. Isolation and identification of flavonoid compounds of Mentha aquatica L. herb. Rocz. Chem. 1977, 51, 701.

175. Voirin, B.; Bayet, C.; Faure, O.; Jullien, F. Free flavonoid aglycones as markers of parentage in Mentha aquatica, M. citrata, M. spicata and M. $\times$ piperita. Phytochemistry 1999, 50, 1189-1193. [CrossRef]

176. Shimizu, S.; Karasawa, D.; Ikeda, N. A new Mint (variety of Mentha aquatica L.) conatining (-)-isopinocamphone as a major constituent of essential oil. Agric. Biol. Chem. 1966, 30, 200-201.

177. Mkaddem, M.; Bouajila, J.; Ennaiar, M.; Lebrihi, A.; Mathieu, F.; Romdhane, M. Chemical composition and antimicrobial and antioxidant activities of Mentha (longifolia L. and viridis) essential oils. J. Food Sci. 2009, 74, M358. [CrossRef]

178. Bourwieg, D.; Pohl, R. Flavonoids from Mentha longifolia. Planta Med. 1973, 24, 304. [CrossRef] [PubMed]

179. Monfared, A.; Nabid, M.R.; Rustaiyan, A. Composition of a carvone chemotype of Mentha longifolia (L.) Huds. from Iran. J. Essent. Oil Res. 2002, 14, 51-52. [CrossRef]

180. Ghoulami, S.; Il Idrissi, A.; Fkih-Tetouani, S. Phytochemical study of Mentha longifolia of Morocco. Fitoterapia 2001, 72, 596-598. [CrossRef]

181. Salman, M.; Abdel-Hameed, E.-S.S.; Bazaid, S.A.; Dabi, M.M. Chemical composition for hydrodistillation essential oil of Mentha longifolia by gas chromatography-mass spectrometry from north regions in Kingdom of Saudi Arabia. Pharma Chem. 2015, 7, 34-40.

182. Sharaf, M.; El-Ansari, M.A.; Saleh, N.A.M. Flavone glycosides from Mentha longifolia. Fitoterapia 1999, 70, 478-483. [CrossRef]

183. Okoh, O.O.; Afolayan, A.J. The effects of hydrodistillation and solvent free microwave extraction methods on the chemical composition and toxicity of essential oils from the leaves of Mentha longifolia L. subsp. capensis. Afr. J. Pharm. Pharmacol. 2011, 5, 2474-2478. [CrossRef]

184. Oyedeji, A.O.; Afolayan, A.J. Chemical composition and antibacterial activity of the essential oil isolated from South African Mentha longifolia (L.) L. subsp. capensis (Thunb.). Briq. J. Essent. Oil Res. 2006, 18, 57-59. [CrossRef]

185. Pektar, S.Y. The Composition, Geographical Variation and Antimicrobial Activity of Mentha longifolia subsp. polyadena (Lamiaceae) Leaf Essential Oils. Ph.D. Thesis, University of the Witswatersrand, Johannesburg, South Africa, 2006.

186. Viljoen, A.M.; Petkar, S.; Van Vuuren, S.F.; Figueiredo, A.C.; Pedro, L.G.; Barroso, J.G. The chemo-geographical variation in essential oil composition and the antimicrobial properties of "wild mint"-Mentha longifolia subsp. polyadena (Lamiaceae) in Southern Africa. J. Essent. Oil Res. 2006, 18, 60-65. [CrossRef]

187. Mahboubi, M.; Haghi, G. Antimicrobial activity and chemical composition of Mentha pulegium L. essential oil. J. Ethnopharmacol. 2008, 119, 325-327. [CrossRef]

188. Fatiha, B.; Didier, H.; Naima, G.; Khodir, M.; Martin, K.; Léocadie, K.; Caroline, S.; Mohamed, C.; Pierre, D. Phenolic composition, in vitro antioxidant effects and tyrosinase inhibitory activity of three Algerian Mentha species: M. spicata (L.), M. pulegium (L.) and M. rotundifolia (L.) Huds (Lamiaceae). Ind. Crops Prod. 2015, 74, 722-730. [CrossRef] 
189. Teixeira, B.; Marques, A.; Ramos, C.; Batista, I.; Serrano, C.; Matos, O.; Neng, N.R.; Nogueira, J.M.F.; Saraiva, J.A.; Nunes, M.L. European pennyroyal (Mentha pulegium) from Portugal: Chemical composition of essential oil and antioxidant and antimicrobial properties of extracts and essential oil. Ind. Crops Prod. 2012, 36, 81-87. [CrossRef]

190. Gülçin, İ.; Gören, A.C.; Taslimi, P.; Alwasel, S.H.; Kılıc, O.; Bursal, E. Anticholinergic, antidiabetic and antioxidant activities of Anatolian pennyroyal (Mentha pulegium)-analysis of its polyphenol contents by LC-MS/MS. Biocatal. Agric. Biotechnol. 2020, 23. [CrossRef]

191. Mallavarapu, G.R.; Ramesh, S.; Subrahmanyam, K. Composition of the essential oil of Micromeria biflora. J. Essent. Oil Res. 1997, 9 , 23-26. [CrossRef]

192. Moghadam, S.E.; Ebrahimi, S.N.; Gafner, F.; Ochola, J.B.; Marubu, R.M.; Lwande, W.; Haller, B.F.; Salehi, P.; Hamburger, M. Metabolite profiling for caffeic acid oligomers in Satureja biflora. Ind. Crops Prod. 2015, 76, 892-899. [CrossRef]

193. Matasyoha, J.C.; Kiplimoa, J.J.; Karubiub, N.M.; Hailstorksc, T.P. Chemical composition and antimicrobial activity of Satureja biflora (Lamiaceae). Bull. Chem. Soc. Ethiop. 2007, 21, 249-254. [CrossRef]

194. Chowdhury, T.; Mandal, A.; Roy, S.C.; De Sarker, D. Diversity of the genus Ocimum (Lamiaceae) through morpho-molecular (RAPD) and chemical (GC-MS) analysis. J. Genet. Eng. Biotechnol. 2017, 15, 275-286. [CrossRef]

195. Thappa, R.K.; Bhatia, M.S.; Aggarwal, S.G.; Dhar, K.L.; Atal, C.K. Ocimin, a novel neolignan from Ocimum americanum. Phytochemistry 1979, 18, 9422. [CrossRef]

196. Djibo, A.K.; Samate, A.D.; Nacro, M. Chemical composition of the essential oil of Ocimum americanum Linn., syn. Ocimum. canum Sims from Burkina Faso. Comptes Rendus Chim. 2004, 7, 1033-1037. [CrossRef]

197. Upadhyay, R.K.; Misra, L.N.; Singh, G. Sesquiterpene alcohols of the copane series from essential oil of Ocimum americanum. Phytochemistry 1991, 30, 691-693. [CrossRef]

198. Matasyoh, J.C.; Bendera, M.M.; Ogendo, J.O.; Omollo, E.O.; Deng, A.L. Volatile leaf oil constituents of Ocimum americanum L. occurring in western Kenya. Bull. Chem. Soc. Ethiop. 2006, 20, 177-180. [CrossRef]

199. Vieira, R.F.; Grayer, R.J.; Paton, A.J. Chemical profiling of Ocimum americanum using external flavonoids. Phytochemistry 2003, 63, 555-567. [CrossRef]

200. Shadia, E.; El-Aziz, A.; Omer, E.A.; Sabra, A.S. Chemical composition of Ocimum americanum essential oil and its biological effects against Agrotis ipsilon, (Lepidoptera: Noctuidae). Res. J. Agric. Biol. Sci. 2007, 3, 740-747.

201. Sishu, R.; Taddesse, S.; Bucar, F.; Asres, K. Chemical composition and antioxidant activity of the essential oils of Ocimum americanum and Ocimum basillicum var. thyrsiflorum. Int. J. Essent. Oil Ther. 2010, 4, 64-68.

202. Padalia, R.C.; Verma, R.S.; Chauhan, A. Diurnal variations in aroma profile of Ocimum basilicum L., O. gratissimum L., O. americanum L., and O. kilimandscharicum Guerke. J. Essent. Oil Res. 2017, 29, 248-261. [CrossRef]

203. Vieira, P.R.N.; De Morais, S.M.; Bezerra, F.H.Q.; Travassos, P.A.; Oliveira, Í.R.; Silva, M.G.V. Chemical composition and antifungal activity of essential oils from Ocimum species. Ind. Crops Prod. 2014, 55, 267-271. [CrossRef]

204. Hussein, A.A.; Meyer, J.J.M.; Jimeno, M.L.; Rodríguez, B. Bioactive diterpenes from Orthosiphon labiatus and Salvia africana-lutea. J. Nat. Prod. 2007, 70, 293-295. [CrossRef]

205. Kapewangolo, P.; Omolo, J.J.; Fonteh, P.; Kandawa-Schulz, M.; Meyer, D. Triterpenoids from Ocimum labiatum activates latent HIV-1 expression in vitro: Potential for use in adjuvant therapy. Molecules 2017, 22, 1703. [CrossRef] [PubMed]

206. Naidoo, Y.; Sadashiva, C.T.; Kasim, N.; Nicholas, A.; Naidoo, G. Chemical Composition and Antimicrobial Activity of the Essential Oil of Ocimum obovatum E. Mey. Ex Benth. (Lamiaceae). J. Essent. Oil Bear. Plants 2014, 17, 142-147. [CrossRef]

207. Burger, I.; Burger, B.V.; Albrecht, C.F.; Spies, H.S.C.; Sandor, P. Triterpenoid saponins from Becium grandiflorum var. obovatum. Phytochemistry 1998, 49, 2087-2095. [CrossRef]

208. Sundarammal, S.; Thirugnanasampandan, R.; Selvi, M.T. Chemical composition analysis and antioxidant activity evaluation of essential oil from Orthosiphon thymiflorus (Roth) Sleesen. Asian Pac. J. Trop. Biomed. 2012, 2, S112-S115. [CrossRef]

209. François, T.; Michel, J.D.P.; Vyry, W.N.A.; Fabrice, F.B.; Lambert, S.M.; Henri, A.Z.P.; Chantal, M. Composition and antifungal properties of essential oils from five plants growing in the mountainous area of the West Cameroon. J. Essent. Oil Bear. Plants 2013, 16, 679-688. [CrossRef]

210. Hussein, A.A. Chemistry of South African Lamiaceae: Structures and biological activity of terpenoids. In Terpenes and Terpenoids; Perveen, S., Al-Taweel, A., Eds.; IntechOpen: London, UK, 2018; pp. 13-38.

211. Ngassapa, O.D.; Runyoro, D.K.B.; Vagionas, K.; Graikou, K.; Chinou, I.B. Chemical composition and antimicrobial activity of Geniosporum rotundifolium Briq and Haumaniastrum villosum (Bene) AJ Paton (Lamiaceae) essential oils from Tanzania. Trop. J. Pharm. Res. 2016, 15, 107-113. [CrossRef]

212. Liu, G.; Ruedi, P. Phyllocladanes (13B-Kauranes) from Plectranthus ambiguus. Phytochemistry 1996, 41, $1563-1568$.

213. Viljoen, A.M.; Demirci, B.; Başer, K.H.C.; Potgieter, C.J.; Edwards, T.J. Microdistillation and essential oil chemistry-A useful tool for detecting hybridisation in Plectranthus (Lamiaceae). S. Afr. J. Bot. 2006, 72, 99-104. [CrossRef]

214. Stavri, M.; Paton, A.; Skelton, B.W.; Gibbons, S. Antibacterial diterpenes from Plectranthus ernstii. J. Nat. Prod. 2009, 72, 1191-1194. [CrossRef]

215. Fournier, G.; Paris, M.; Dumitresco, S.M.; Pages, N.; Boudene, C. Contribution to the study of Plectranthus fruticosus leaf essential oil. Planta Med. 1986, 52, 486-488. [CrossRef]

216. Gaspar-Marques, C.; Simões, M.F.; Duarte, A.; Rodríguez, B. Labdane and kaurane diterpenoids from Plectranthus fruticosus. J. Nat. Prod. 2003, 66, 491-496. [CrossRef] 
217. Katti, S.B.; Rueedi, P.; Eugster, C.H. Diterpenoid quinomethans, vinylogous quinones and a phyllocladene derivative from Plectranthus purpuratus Harv. (Labiatae). Helv. Chim. Acta 1982, 65, 2189. [CrossRef]

218. Simões, M.F.; Rijo, P.; Duarte, A.; Barbosa, D.; Matias, D.; Delgado, J.; Cirilo, N.; Rodríguez, B. Two new diterpenoids from Plectranthus species. Phytochem. Lett. 2010, 3, 221-225. [CrossRef]

219. Wellsow, J.; Grayer, R.J.; Veitch, N.C.; Kokubun, T.; Lelli, R.; Kite, G.C.; Simmonds, M.S.J. Insect-antifeedant and antibacterial activity of diterpenoids from species of Plectranthus. Phytochemistry 2006, 67, 1818-1825. [CrossRef]

220. Mota, A.H.; Sousa, A.; Figueira, M.; Amaral, M.; Sousa, B.; Rocha, J.; Fattal, E.; Almeida, A.J.; Reis, C.P. Chapter 19-Naturalbased consumer health nanoproducts: Medicines, cosmetics, and food supplements. In Micro and Nano Technologies; Elsevier: Amsterdam, The Netherlands, 2020; pp. 527-578, ISBN 978-0-12-816787-8.

221. Brito, E.; Gomes, E.; Falé, P.L.; Borges, C.; Pacheco, R.; Teixeira, V.; Machuqueiro, M.; Ascensão, L.; Serralheiro, M.L.M. Bioactivities of decoctions from Plectranthus species related to their traditional use on the treatment of digestive problems and alcohol intoxication. J. Ethnopharmacol. 2018, 220, 147-154. [CrossRef]

222. Narukawa, Y.; Shimizu, N.; Shimotohno, K.; Takeda, T. Two new diterpenoids from Plectranthus nummularius Briq. Chem. Pharm. Bull. 2001, 49, 1182-1184. [CrossRef]

223. Lamiaceae, L.; Lawson, S.K.; Sharp, L.G.; Satyal, P.; Setzer, W.N. Volatile components of the aerial parts of Prunella vulgaris L. (Lamiaceae). Am. J. Essent. Oils Nat. Prod. 2020, 8, 17-19.

224. Gu, X.; Li, Y.; Mu, J.; Zhang, Y. Chemical constituents of Prunella vulgaris. J. Environ. Sci. 2013, 25, S161-S163. [CrossRef]

225. Chen, Y.; Guo, Q.; Zhu, Z.; Zhang, L.; Dai, X. Comparative analysis of the essential oil of flowers, leaves and stems of Prunella vulgaris L. J. Essent. Oil Bear. Plants 2012, 15, 662-666. [CrossRef]

226. Kojima, H.; Ogura, H. Triterpenoids from Prunella vulgaris. Phytochemistry 1986, 25, 729-733. [CrossRef]

227. Kojima, H.; Tominaga, H.; Sato, S.; Takayanagi, H.; Ogura, H. Two novel hexacyclic triterpenoids from Prunella vulgaris. Phytochemistry 1988, 27, 2921-2925. [CrossRef]

228. Lou, H.Y.; Jin, L.; Huang, T.; Wang, D.P.; Liang, G.Y.; Pan, W.D. Vulgarisins B-D, three novel diterpenoids with a rare skeleton isolated from Prunella vulgaris Linn. Tetrahedron Lett. 2017, 58, 401-404. [CrossRef]

229. Wang, S.-J.; Wang, X.-H.; Dai, Y.-Y.; Ma, M.-H.; Rahman, K.; Nian, H.; Zhang, H. Prunella vulgaris: A comprehensive review of chemical constituents, pharmacological effects and clinical applications. Curr. Pharm. Des. 2019. [CrossRef]

230. Davies-Coleman, M.T.; Rivett, D.E.A. Transformation of Hispanolone from Ballota africana into 15,16-epoxy-9-hydroxylabda13(16),14-diene. S. Afr. J. Chem. 1990, 43, 117-119.

231. Davies-Coleman, M.T. Extractives from Six Species of Lamiaceae. Ph.D. Thesis, Rhodes University, Grahamstown, South Africa, 1987.

232. Gray, C.A.; Rivett, D.E.A.; Davies-Coleman, M.T. The absolute stereochemistry of a diterpene from Ballota aucheri. Phytochemistry 2003, 63, 409-413. [CrossRef]

233. Bashwira, S.; Hootele, C.; Tourwe, D.; Pepermans, H.; Laus, G.; Van Binst, G. Cleromyrine I, a new cyclohexapeptide from Clerodendrum myricoides. Tetrahedron 1989, 45, 5845. [CrossRef]

234. Msonthi, J.; Hostettmann, K.; Maillard, M. Phytochemical studies of medicinal plants from Malawi. In Chemistry, Biological and Pharmacological Properties of African Medicinal Plants; Hostettmann, K., Chinyanganya, F., Maillard, M., Wolfender, J.-L., Eds.; University of Zimbabwe Publications: Harare, Zimbabwe, 1996.

235. Toyota, M.; Msonthi, J.D.; Hostettmann, K. A molluscicidal and antifungal triterpenoid saponin from the roots of Clerodendrum wildii. Phytochemistry 1990, 29, 2849-2851. [CrossRef]

236. De Martino, L.; Roscigno, G.; Mancini, E.; De Falco, E.; De Feo, V. Chemical composition and antigerminative activity of the essential oils from five Salvia species. Molecules 2010, 15, 735-746. [CrossRef] [PubMed]

237. Afonso, A.F.; Pereira, O.R.; Fernandes, Â.; Calhelha, R.C.; Silva, A.M.S.; Ferreira, R.C.F.; Cardoso, S.M. Phytochemical composition and bioactive effects of Salvia africana, Salvia officinalis "Icterina" and Salvia mexicana aqueous Extracts. Molecules 2019, $24,4327$. [CrossRef] [PubMed]

238. Fisher, V.L. Indigenous Salvia Species-An Investigation of the Antimicrobial Activity, Antioxidant Activity and Chemical Composition of Leaf Extracts. Ph.D. Thesis, University of the Witswatersrand, Johannesburg, South Africa, 2005.

239. Kamatou, G.P.P.; Van Zyl, R.L.; Van Vuuren, S.F.; Viljoen, A.M.; Figueiredo, A.C.; Barroso, J.G.; Pedro, L.G.; Tilney, P.M. Chemical composition, leaf trichome types and biological activities of the essential oils of four related Salvia species indigenous to Southern Africa. J. Essent. Oil Res. 2006, 18, 72-79. [CrossRef]

240. Kamatou, G.P.P.; Viljoen, A.M.; Steenkamp, P. Antioxidant, antiinflammatory activities and HPLC analysis of South African Salvia species. Food Chem. 2010, 119, 684-688. [CrossRef]

241. Kamatou, G.P.P.; Van Zyl, R.L.; Van Vuuren, S.F.; Figueiredo, A.C.; Barroso, J.G.; Pedro, L.G.; Viljoen, A.M. Seasonal variation in essential oil composition, oil toxicity and the biological activity of solvent extracts of three South African Salvia species. S. Afr. J. Bot. 2008, 74, 230-237. [CrossRef]

242. Kamatou, G.P.P.; Viljoen, A.M.; Figueiredo, A.C.; Tilney, P.M.; Van Zyl, R.L.; Barroso, J.G.; Pedro, L.G.; Van Vuuren, S.F. Trichomes, essential oil composition and biological activities of Salvia albicaulis Benth. and S. dolomitica Codd, two species from the Cape region of South Africa. S. Afr. J. Bot. 2007, 73, 102-108. [CrossRef]

243. Arief, M.M.H.; Abdel, A.; Hussein, F.; Mohammed, A.; Elmwafy, H.M. Chemical and Bioactivity Studies on Salvia africana-lutea: Cytotoxicity and Apoptosis Induction by Abietane Diterpenes Isolated from Salvia africana-lutea. J. Basic Environ. Sci. 2018, 5, 72-79. 
244. Glyphis, J.P.; Puttick, G.M. Phenolics in some southern African Mediterranean shrubland plants. Phytochemistry 1988, $27,743-751$. [CrossRef]

245. Lim Ah Tock, M.J.; Kamatou, G.P.P.; Combrinck, S.; Sandasi, M.; Viljoen, A.M. A chemometric assessment of essential oil variation of three Salvia species indigenous to South Africa. Phytochemistry 2020, 172, 112249. [CrossRef]

246. Kamatou, G.P.P.; Van Vuuren, S.F.; Van Heerden, F.R.; Seaman, T.; Viljoen, A.M. Antibacterial and antimycobacterial activities of South African Salvia species and isolated compounds from S. chamelaeagnea. S. Afr. J. Bot. 2007, 73, 552-557. [CrossRef]

247. Onayade, O.A.; Scheffer, J.J.C.; Svendsen, A.B. Polynuclear aromatic compounds and other constituents of the herb essential oil of Salvia coccinea Juss. ex Murr. Flavour Fragr. J. 1991, 6, 281-289. [CrossRef]

248. Grzeszczuk, M.; Salachna, P.; Meller, E. Changes in photosynthetic pigments, total phenolic content, and antioxidant activity of Salvia coccinea Buc'hoz Ex Etl. induced by exogenous salicylic acid and soil salinity. Molecules 2018, 23, 1296. [CrossRef]

249. Abreu, M.E.; Muller, M.; Alegre, L.; Munne-Bosch, S. Phenolic diterpene and $\alpha$-tocopherol contents in leaf extracts of 60 Salvia species. J. Sci. Food Agric. 2008, 88, 2648-2653. [CrossRef]

250. Bassolino, L.; Giacomelli, E.; Giovanelli, S.; Pistelli, L.; Cassetti, A.; Damonte, G.; Bisio, A.; Ruffoni, B. Tissue culture and aromatic profile in Salvia dolomitica Codd. Plant Cell. Tissue Organ Cult. 2015, 121, 83-95. [CrossRef]

251. Caser, M.; Chitarra, W.; D'Angiolillo, F.; Perrone, I.; Demasi, S.; Lovisolo, C.; Pistelli, L.; Pistelli, L.; Scariot, V. Drought stress adaptation modulates plant secondary metabolite production in Salvia dolomitica Codd. Ind. Crops Prod. 2019, 129, 85-96. [CrossRef]

252. Ebani, V.V.; Nardoni, S.; Bertelloni, F.; Giovanelli, S.; Ruffoni, B.; D’Ascenzi, C.; Pistelli, L.; Mancianti, F. Activity of Salvia dolomitica and Salvia somalensis essential oils against bacteria, molds and yeasts. Molecules 2018, 23, 396. [CrossRef] [PubMed]

253. Kamatou, G.P.P.; Van Zyl, R.L.; Van Vuuren, S.F.; Figueiredo, A.C.; Barroso, J.G.; Pedro, L.G. Biological activities and composition of Salvia muirii L. Bol. essential oil. J. Essent. Oil Res. 2006, 18, 48-51. [CrossRef]

254. Grierson, D.S.; Afolayan, A.J. Antibacterial activity of the extracts and the essential oil from the shoots of Salvia namaensis Schinz. S. Afr. J. Sci. 2005, 101, 507-510.

255. Gono-Bwalya, A. The Chemotaxonomy and Biological Activity of Salvia stenophylla (Lamiaceae) and Related Taxa. Ph.D. Thesis, University of the Witwatersrand, Johannesburg, South Africa, 2003.

256. Kamatou, G.P.P.; Viljoen, A.M.; Gono-Bwalya, A.B.; Van Zyl, R.L.; Van Vuuren, S.F.; Lourens, A.C.U.; Başer, K.H.C.; Demirci, B.; Lindsey, K.L.; Van Staden, J.; et al. The in vitro pharmacological activities and a chemical investigation of three South African Salvia species. J. Ethnopharmacol. 2005, 102, 382-390. [CrossRef]

257. Mokoka, T.A.; Peter, X.K.; Fouche, G.; Moodley, N.; Adams, M.; Hamburger, M.; Kaiser, M.; Brun, R.; Maharaj, V.; Koorbanally, N. Antileishmanial activity of 12-methoxycarnosic acid from Salvia repens Burch. ex Benth. (Lamiaceae). S. Afr. J. Bot. 2014, 90, 93-95. [CrossRef]

258. Viljoen, A.M.; Gono-Bwalya, A.; Kamatou, G.P.P.; Başer, K.H.C.; Demirci, B. The essential oil composition and chemotaxonomy of Salvia stenophylla and its allies S. repens and S. runcinata. J. Essent. Oil Res. 2006, 18, 37-45. [CrossRef]

259. Mokoka, T.A. The Discovery and Characterization of Antiprotozoal Compounds from South African Medicinal Plants by a HPLC-Based Activity Profiling Technique. Ph.D. Thesis, University of KwaZulu-Natal, Durban, South Africa, 2013.

260. Figlan, S. Generation of Clonal Microplants and Hairt Root Cultures of the Aromatic Medicinal Plant Salvia runcinata L.f. Ph.D. Thesis, Stellenbosch University, Stellenbosch, South Africa, 2012.

261. Sandasi, M.; Kamatou, G.P.P.; Viljoen, A.M. An untargeted metabolomic approach in the chemotaxonomic assessment of two Salvia species as a potential source of $\alpha$-Bisabolol. Phytochemistry 2012, 84, 94-101. [CrossRef]

262. Wollenweber, E.; Dörr, M.; Rustaiyan, A.; Roitman, J.N.; Graven, E.H. Exudate flavonoids of some Salvia and a Trichostema species. Zeitschrift Naturforsch. Sect. C J. Biosci. 1992, 47, 782-784. [CrossRef]

263. Jequier, C.; Nicollier, G.; Tabacchi, R.; Garnero, J. Constituents of the essential oil of Salvia stenophylla. Phytochemistry 1980, 19, 461-462. [CrossRef]

264. Canzoneri, M.; Bruno, M.; Rosselli, S.; Russo, A.; Cardile, V.; Formisano, C.; Rigano, D.; Senatore, F. Chemical composition and biological activity of Salvia verbenaca essential oil. Nat. Prod. Commun. 2011, 6, 1023-1026. [CrossRef]

265. Russo, A.; Cardile, V.; Graziano, A.C.E.; Formisano, C.; Rigano, D.; Canzoneri, M.; Bruno, M.; Senatore, F. Comparison of essential oil components and in vitro anticancer activity in wild and cultivated Salvia verbenaca. Nat. Prod. Res. 2015, 29, 1630-1640. [CrossRef]

266. Kostić, M.; Zlatković, B.; Miladinović, B.; Živanović, S.; Mihajilov-Krstev, T.; Pavlović, D.; Kitić, D. Rosmarinic acid levels, phenolic contents, antioxidant and antimicrobial activities of the extracts from Salvia verbenaca L. obtained with different solvents and procedures. J. Food Biochem. 2015, 39, 199-208. [CrossRef]

267. Pitarokili, D.; Tzakou, O.; Loukis, A. Essential oil composition of Salvia verticillata, S. verbenaca, S. glutinosa and S. candidissima growing wild in Greece. Flavour Fragr. J. 2006, 21, 670-673. [CrossRef]

268. Chorianopoulos, N.; Evergetis, E.; Mallouchos, A.; Kalpoutzakis, E.; Nychas, G.J.; Haroutounian, S.A. Characterization of the essential oil volatiles of Satureja thymbra and Satureja parnassica: Influence of harvesting time and antimicrobial activity. J. Agric. Food Chem. 2006, 54, 3139-3145. [CrossRef]

269. Skendi, A.; Irakli, M.; Chatzopoulou, P. Analysis of phenolic compounds in Greek plants of Lamiaceae family by HPLC. J. Appl. Res. Med. Aromat. Plants 2017, 6, 62-69. [CrossRef] 
270. Scott, G.; Springfield, E.P.; Coldrey, N. A pharmacognostical study of 26 South African plant species used as traditional medicines. Pharm. Biol. 2004, 42, 186-213. [CrossRef]

271. Collett, L.A.; Davies-Coleman, M.T.; Rivett, D.E.A. 5,6-Dihydro-Pyrones From Syncolostemon argenteus. Phytochemistry 1998, 48, 651. [CrossRef]

272. Marthe, D.C.Z.; Fidèle, M.A.; Fernand, G.; Mansourou, M. Chemical composition and in vitro investigation of biological activities of Hemizygia bracteosa (Benth.) Briq leaves. J. Pharmacogn. Phyther. 2018, 10, 11-20. [CrossRef]

273. Davies-Coleman, M.T.; Rivett, D.E.A. An $\alpha$-pyrone from Syncolostemon densiflorus. Phytochemistry 1994, 35, 1590-1592. [CrossRef]

274. Bruce, T.J.A.; Birkett, M.A.; Blande, J.; Hooper, A.M.; Martin, J.L.; Khambay, B.; Prosser, I.; Smart, L.E.; Wadhams, L.J. Response of economically important aphids to components of Hemizygia petiolata essential oil. Pest Manag. Sci. 2005, 61, 1115-1121. [CrossRef] [PubMed]

275. Davies-Coleman, M.T.; Rivett, D.E.A. Stereochemical studies on boronolide, an $\alpha$-pyrone from Tetradenia barberae. Phytochemistry 1987, 26, 3047-3050. [CrossRef]

276. Baldin, V.P.; de Scodro, R.B.L.; Lopes-Ortiz, M.A.; de Almeida, A.L.; Gazim, Z.C.; Ferarrese, L.; Faiões, V.d.S.; Torres-Santos, E.C.; Pires, C.T.A.; Caleffi-Ferracioli, K.R.; et al. Anti-Mycobacterium tuberculosis activity of essential oil and 6,7-dehydroroyleanone isolated from leaves of Tetradenia riparia (Hochst.) Codd (Lamiaceae). Phytomedicine 2018, 47, 34-39. [CrossRef] [PubMed]

277. Blythe, E.K.; Tabanca, N.; Demirci, B.; Kendra, P.E. Chemical composition of essential oil from Tetradenia riparia and its attractant activity for Mediterranean fruit fly, Ceratitis capitata. Nat. Prod. Commun. 2020, 15. [CrossRef]

278. Godoy, R.L.O.; Koketsu, M.; Goncalves, S.L.; Lopes, D.; Sa Sobrinho, A.F.; Torquilho, H.S. Essential oil of Moschosma riparium Hochst. (Lamiaceae) from Manaus, Amazonas, Brazil. J. Essent. Oil Res. 1999, 11, 321-323. [CrossRef]

279. Milato, J.V.; Silva, R.S.F.; Figueiredo, F.d.S.; Azevedo, D.d.A.; Ribeiro, C.A.B.; Leitão, G.G. Use of counter-current chromatography as a selective extractor for the diterpenequinone $7 \alpha$-hydroxyroyleanone from Tetradenia riparia. J. Chromatogr. A 2018, 1537, 135-140. [CrossRef]

280. Hannweg, K.; Visser, G.; de Jager, K.; Bertling, I. In vitro-induced polyploidy and its effect on horticultural characteristics, essential oil composition and bioactivity of Tetradenia riparia. S. Afr. J. Bot. 2016, 106, 186-191. [CrossRef]

281. Van Puyvelde, L.; Dube, S.; Uwimana, E.; Uwera, C.; Dommisse, R.A.; Esmans, E.L.; Van Schoor, O.; Vlietinck, A.J. New $\alpha$-pyrones from Iboza riparia. Phytochemistry 1979, 18, 1215-1218. [CrossRef]

282. Van Puyvelde, L.; De Kimpe, N.; Dubé, S.; Chagnon-Dubé, M.; Boily, Y.; Borremans, F.; Schamp, N.; Anteunis, M.J.O. 1' '2' Didecacetylboronolide, an $\alpha$-Pyrone from Iboza riparia. Phytochemistry 1981, 20, 2753-2755. [CrossRef]

283. Tokuda, M.; Kurogome, Y.; Katoh, R.; Nohara, Y.; Hattori, Y.; Makabe, H. Synthesis of four diastereomers and structural revision of tetradenolide. Tetrahedron Lett. 2014, 55, 4189-4192. [CrossRef]

284. Van Puyvelde, L.; Nyirankuriza, S.; Panaebianco, R.; Boily, Y.; Geizer, I.; Sebikali, B.; De Kimpe, N.; Schamp, N. Active principles

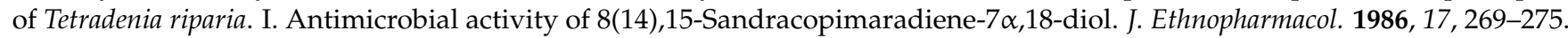
[CrossRef]

285. Van Puyvelde, L.; Liu, M.; Veryser, C.; De Borggraeve, W.M.; Mungarulire, J.; Mukazayire, M.J.; Luyten, W. Active principles of Tetradenia riparia. IV. Anthelmintic activity of 8(14),15-sandaracopimaradiene-7 $\alpha, 18$-diol. J. Ethnopharmacol. 2018, 216, $229-232$. [CrossRef]

286. Van Puyvelde, L.; De Kimpe, N.; Borremans, F.; Zhang, W.; Schamp, N. 8(14),15-Sandaracopimaradiene-2 $\alpha, 18-d i o l$, a minor constituent of the Rwandese medicinal plant Tetradenia riparia. Phytochemistry 1987, 26, 493. [CrossRef]

287. Van Puyvelde, L.; Lefebvre, R.; Mugabo, P.; De Kimpe, N.; Schamp, N. Active principles of Tetradenia riparia; II. Antispasmodic activity of 8(14),15-sandaracopimaradiene-7 $\alpha, 18$-diol. Planta Med. 1987, 53, 156. [CrossRef]

288. Zelnik, R.; Rabenhorst, E.; Matida, A.K.; Gottlier, H.E.; Lavie, D.; Panizza, S. Ibozol, a new Diterpenoid from Iboza riparia. Phytochemistry 1978, 17, 1795-1797. [CrossRef]

289. Ruiters, A.K.; Tilney, P.M.; Van Vuuren, S.F.; Viljoen, A.M.; Kamatou, G.P.P.; Van Wyk, B.-E. The anatomy, ethnobotany, antimicrobial activity and essential oil composition of southern African species of Teucrium (Lamiaceae). S. Afr. J. Bot. 2016, 102, 175-185. [CrossRef]

290. Davies-Coleman, M.T.; Hanson, J.R.; Rivett, D.E.A. Teutrifidin, A neo-clerodane diterpenoid from Teucrium trifidum. Phytochemistry 1994, 36, 1549-1550. [CrossRef]

291. Cabral, C.; Gonçalves, M.J.; Cavaleiro, C.; Salgueiro, L.; Antunes, T.; Sevinate-Pinto, I.; Sales, F. Vitex ferruginea Schumach. Et. Thonn subsp. amboniensis (Gürke) Verdc: Glandular trichomes micromorphology, composition and antifungal activity of the essential oils. J. Essent. Oil Res. 2008, 20, 86-90. [CrossRef]

292. Nyiligira, E.; Viljoen, A.M.; Başer, K.H.C.; Ózek, T.; Van Vuuren, S.F. Essential oil composition and in vitro antimicrobial and anti-inflammatory activity of South African Vitex species. S. Afr. J. Bot. 2004, 70, 611-617. [CrossRef]

293. Suksamrarn, A.; Werawattanametin, K.; Brophy, J.J. Variation of essential oil constituents in Vitex trifolia species. Flavour Fragr. J. 1991, 6, 97-99. [CrossRef]

294. Nair, A.G.R.; Ramesh, P.; Subramanian, S.S. Two unusual flavones (artemetin and 7-desmethyl artemetin) from the leaves of Vitex trifolia. Curr. Sci. 1975, 44, 214-216.

295. Ono, M.; Sawamura, H.; Ito, Y.; Mizuki, K.; Nohara, T. Diterpenoids from the fruits of Vitex trifolia. Phytochemistry 2000, 55, 873-877. [CrossRef] 
296. Masevhe, N.A.; Awouafack, M.D.; Ahmed, A.S.; McGaw, L.J.; Eloff, J.N. Clerodendrumic Acid, a new triterpenoid from Clerodendrum glabrum (Verbenaceae), and antimicrobial activities of fractions and constituents. ChemInform 2014, 45. [CrossRef]

297. Teclegeorgish, Z.W.; Mokgalaka, N.S.; Vukea, N.; de la Mare, J.A.; Tembu, V.J. Cytotoxicity of triterpenoids from Clerodendrum glabrum against triple negative breast cancer cells in vitro. S. Afr. J. Bot. 2020, 133, 144-150. [CrossRef]

298. Wahba, H.M.; Abouzid, S.F.; Sleem, A.A.; Apers, S.; Pieters, L.; Shahat, A.A. Chemical and biological investigation of some Clerodendrum species cultivated in Egypt. Pharm. Biol. 2011, 49, 66-72. [CrossRef] [PubMed]

299. O'Connor, S.E. 1.25-Alkaloids. In Comprehensive Natural Products II; Liu, H.-W., Mander, L.B.T.-C.N.P.I.I., Eds.; Elsevier: Oxford, UK, 2010; pp. 977-1007, ISBN 978-0-08-045382-8.

300. Wansi, J.D.; Devkota, K.P.; Tshikalange, E.; Kuete, V. Alkaloids from the Medicinal Plants of Africa; Elsevier Inc.: Amsterdam, The Netherlands, 2013; ISBN 9780124059276.

301. Harborne, J.B. Methods of Plant Analysis BT_Phytochemical Methods: A Guide to Modern Techniques of Plant Analysis; Harborne, J.B., Ed.; Springer Netherlands: Dordrecht, The Netherlands, 1984; ISBN 978-94-009-5570-7.

302. Matos, M.J.; Santana, L.; Uriarte, E.; Abreu, O.A.; Yordi Molina, E.; Guardado, E. Coumarins-An Important Class of Phytochemicals. In Phytochemicals-Isolation, Characterisation and Role in Human Health; Rao, V., Rao, L., Eds.; IntechOpen: London, UK, 2015; ISBN 9781626239777.

303. Faridi Esfanjani, A.; Assadpour, E.; Jafari, S.M. Improving the bioavailability of phenolic compounds by loading them within lipid-based nanocarriers. Trends Food Sci. Technol. 2018, 76, 56-66. [CrossRef]

304. Karakaya, S. Bioavailability of phenolic compounds. Crit. Rev. Food Sci. Nutr. 2004, 44, 453-464. [CrossRef]

305. Shahidi, F.; Varatharajan, V.; Oh, W.Y.; Peng, H. Phenolic compounds in agri-food by-products, their bioavailability and health effects. J. Food Bioact. 2019, 5. [CrossRef]

306. Shahidi, F.; Peng, H. Bioaccessibility and bioavailability of phenolic compounds. J. Food Bioact. 2018, 4, 11-68. [CrossRef]

307. Fernandez-Panchon, M.S.; Villano, D.; Troncoso, A.M.; Garcia-Parrilla, M.C. Antioxidant activity of phenolic compounds: From in vitro results to in vivo evidence. Crit. Rev. Food Sci. Nutr. 2008, 48, 649-671. [CrossRef]

308. Chen, G.L.; Chen, S.G.; Zhao, Y.Y.; Luo, C.X.; Li, J.; Gao, Y.Q. Total phenolic contents of 33 fruits and their antioxidant capacities before and after in vitro digestion. Ind. Crops Prod. 2014, 57, 150-157. [CrossRef]

309. Lukhoba, C.W.; Simmonds, M.S.J.; Paton, A.J. Plectranthus: A review of ethnobotanical uses. J. Ethnopharmacol. 2006, 103, 1-24. [CrossRef]

310. Salame, R.; Cheikh-Ali, Z.; Bories, C.; Adiko, M.; Poupon, E.; Champy, P. Pyrone and unusually furanone-substituted flavones from the leaves of Hoslundia opposita. Planta Med. 2012, 78, 1777-1779. [CrossRef]

311. Dorman, H.J.D.; Kosar, M.; Kahlos, K.; Holm, Y.; Hiltunen, R. Antioxidant properties and composition of aqueous extracts from Mentha species, hybrids, varieties, and cultivars. J. Agric. Food Chem. 2003, 51, 4563-4569. [CrossRef]

312. Kumar, T.V.; Reddy, G.V.; Babu, K.S.; Rao, J.M. Stereoselective synthesis of umuravumbolide. Tetrahedron Asymmetry 2013, 24, 594-598. [CrossRef]

313. Demetzos, C.; Dimas, K.S. Labdane-type diterpenes: Chemistry and biological activity. In Bioactive Natural Products (Part F); Elsevier: Amsterdam, The Netherlands, 2001; Volume 25, pp. 235-292, ISBN 1572-5995.

314. Manse, Y.; Ninomiya, K.; Nishi, R.; Hashimoto, Y.; Chaipech, S.; Muraoka, O.; Morikawa, T. Labdane-type diterpenes, Galangalditerpenes A-C, with melanogenesis inhibitory activity from the fruit of Alpinia galanga. Molecules 2017, 22, 2279. [CrossRef]

315. Said, S.A. Antimalarial effect and other properties of Hoslundia opposita-A review. Glob. J. Pharm. Pharm. Sci. 2017, 4, 1-6. [CrossRef]

316. Scandiffio, R.; Geddo, F.; Cottone, E.; Querio, G.; Antoniotti, S.; Pia Gallo, M.; Maffei, M.E.; Bovolin, P. Protective effects of (E)- $\beta$-caryophyllene (BCP) in chronic inflammation. Nutrients 2020, 12, 3273. [CrossRef]

317. Adlof, C.C. How Does Harvesting Impact White Sage (Salvia apiana) as a Cultural Resource in Southern California? Ph.D. Thesis, California State University, Northridge, CA, USA, 2015.

318. Cohen, M.M. Tulsi-Ocimum sanctum: A herb for all reasons. J. Ayurveda Integr. Med. 2014, 5, 251-259. [CrossRef]

319. Khoury, M.; Stien, D.; Eparvier, V.; Ouaini, N.; El Beyrouthy, M. Report on the medicinal use of eleven Lamiaceae species in Lebanon and rationalization of their antimicrobial potential by examination of the chemical composition and antimicrobial activity of their essential oils. Evid. Based Complement. Altern. Med. 2016, 2016, 2547169. [CrossRef]

320. Zhao, T.; Tang, H.; Xie, L.; Zheng, Y.; Ma, Z.; Sun, Q.; Li, X. Scutellaria baicalensis Georgi. (Lamiaceae): A review of its traditional uses, botany, phytochemistry, pharmacology and toxicology. J. Pharm. Pharmacol. 2019, 71, 1353-1369. [CrossRef]

321. Li, M.; Li, Q.; Zhang, C.; Zhang, N.; Cui, Z.; Huang, L.; Xiao, P. An ethnopharmacological investigation of medicinal Salvia plants (Lamiaceae) in China. Acta Pharm. Sin. B 2013, 3, 273-280. [CrossRef]

322. Hu, R.; Lin, C.; Xu, W.; Liu, Y.; Long, C. Ethnobotanical study on medicinal plants used by Mulam people in Guangxi, China. J. Ethnobiol. Ethnomed. 2020, 16, 1-50. [CrossRef] [PubMed]

323. Perrino, E.V.; Perrino, P. Crop Wild Relatives: Know How Past and Present to Improve Future Research, Conservation and Utilization Strategies, Especially in Italy: A Review. Genet. Resour. Crop Evol. 2020, 67, 1067-1105. [CrossRef]

324. Asong, J.A.; Ndhlovu, P.T.; Khosana, N.S.; Aremu, A.O.; Otang-Mbeng, W. Medicinal plants used for skin-related diseases among the Batswanas in Ngaka Modiri Molema District Municipality, South Africa. S. Afr. J. Bot. 2019, 126, 11-20. [CrossRef]

325. Nafuka, S.N.; Mumbengegwi, D.R. Phytochemical analysis and in vitro anti-plasmodial activity of selected ethnomedicinal plants used to treat malaria associated Symptoms in Northern Namibia. Int. Sci. Technol. J. Namibia 2013, 2, 78-93. 
326. Jäger, A.K.; Mohoto, S.P.; Van Heerden, F.R.; Viljoen, A.M. Activity of a traditional South African epilepsy remedy in the GABA-benzodiazepine receptor assay. J. Ethnopharmacol. 2005, 96, 603-606. [CrossRef]

327. Watt, J.M.; Breyer-Brandwijk, M.G. The Medicinal and Poisonous Plants of Southern and Eastern Africa, 2nd ed.; Livingstone: London, UK, 1962; ISBN 0608142948.

328. Moteetee, A.; Moffett, R.O.; Seleteng-Kose, L. A review of the ethnobotany of the Basotho of Lesotho and the Free State Province of South Africa (South Sotho). S. Afr. J. Bot. 2019, 122, 21-56. [CrossRef]

329. Leffers, A. Gemsbok Bean and Kalahari Truffle. Traditional Plant Use by Jul'hoansi in North-Eastern Namibia; Macmillan Education: Windhoek, Namibia, 2003; ISBN 999160491X.

330. Semenya, S.S.; Maroyi, A. Ethnobotanical survey of plants used by Bapedi traditional healers to treat tuberculosis and its opportunistic infections in the Limpopo Province, South Africa. S. Afr. J. Bot. 2019, 122, 401-421. [CrossRef]

331. Pooley, E. A Field Guide to Wildflowers: KwaZulu-Natal and the Eastern Region; Natal Flora Publications Trust: Durban, South Africa, 1999; ISBN 0620215003.

332. Sahrial, I.; Solfaine, R. Coleus amboinicus extract increases transforming growth factor-1 $\beta$ expression in Wistar rats with cisplatininduced nephropathy. Vet. World 2019, 12, 1346-1351. [CrossRef]

333. Thanaseelungkoon, N.; Julsrigival, J.; Phannachet, K.; Chansakaow, S. Chemical compositions and biological activities of essential oils obtained from some Apiaceous and Lamiaceous plants collected in Thailand. Asian Pac. J. Trop. Med. 2018, 11, 486-494. [CrossRef]

334. Musila, F.M.; Nguta, J.; Lukhoba, C.W.; Dossaji, S.F. Antibacterial and antifungal activities of 10 Kenyan Plectranthus species in the Coleus clade. Artic. J. Pharm. Res. 2017, 11, 1-7.

335. Haryani, R.; Harahap, U.; Masfria, M.; Satria, D. Cytoprotective activity of ethanol fraction of Coleus amboinicus Lour. leaves against vero cells induced by $\mathrm{H}_{2} \mathrm{O}_{2}$. Asian J. Pharm. Clin. Res. 2018, 11, 28-30. [CrossRef]

336. Tarigan, M.H.; Harahap, U.; Dalimunthe, A.; Nerdy, N. Antioxidant activity and cardioprotective activity of bangun-bangun leaves (Plectranthus amboinicus Lour.) ethanolic extract. Asian J. Pharm. Clin. Res. 2018, 11, 165-168. [CrossRef]

337. Lipin Dev, D.S.; Menon, D.B. Essential oil extracted from Plectranthus amboinicus induces apoptosis in the lung cancer cells via mitochondrial pathway. Int. J. Pharm. Sci. Drug Res. 2017, 9, 83-89. [CrossRef]

338. Hutchings, A.; Lewis, A.H.; Cunningham, A.B. Zulu Medicinal Plants: An Inventory; University of Natal Press: Pietermaritzburg, South Africa, 1996; ISBN 0869808931.

339. Menon, D.B.; Sasikumar, J.M.; Latha, K. Anti inflammtory and cytotoxic activity of methanolic extract of Plectranthus hadiensis stem. Pharmacologyonline 2011, 3, 275-282.

340. Mogale, M.M.P. The Ethnobotany of Central Sekhukhuneland, South Africa. Master's Thesis, University of Johannesburg, Johannesburg, South Africa, 2018.

341. Mogale, M.M.P.; Raimondo, D.C.; Van Wyk, B.-E. The ethnobotany of Central Sekhukhuneland, South Africa. S. Afr. J. Bot. 2019, 122, 90-119. [CrossRef]

342. Magwede, K. A Quantitative Survey of Traditional Plant Use of the Vhavenda, Limpopo Province, South Africa. Ph.D. Thesis, University of Johannesburg, Johannesburg, South Africa, 2018.

343. Palmer, E. The South African Herbal; Tafelberg: Cape Town, South Africa, 1985; ISBN 0624021777.

344. Maharaj, R.; Maharaj, V.; Crouch, N.R.; Bhagwandin, N.; Folb, P.I.; Pillay, P.; Gayaram, R. Evaluation of selected South African ethnomedicinal plants as mosquito repellents against the Anopheles arabiensis mosquito in a rodent model. Malar. J. 2010, 9, 1-8. [CrossRef]

345. Von Koenen, E. Heil-und Giftplanzen in Sudwestafrika; Akademischer Verlag: Windhoek, Namibia, 1977 ; ISBN 0620028874.

346. Oloyede, A.M.; Akindele, S.K. A preliminary investigation of the acute toxicity of the ethanolic extract of Hoslundia opposita in Mus musculus (Swiss Mice). Herb. Med. J. 2019, 4, 163-170. [CrossRef]

347. Olajide, O.A.; Awe, S.O.; Makinde, J.M. Central nervous system depressant effect of Hoslundia opposita Vahl. Phyther. Res. 1999, 13, 425-426. [CrossRef]

348. Risa, J.; Risa, A.; Adsersen, A.; Gauguin, B.; Stafford, G.I.; Van Staden, J.; Jäger, A.K. Screening of plants used in southern Africa for epilepsy and convulsions in the GABAA-benzodiazepine receptor assay. J. Ethnopharmacol. 2004, 93, 177-182. [CrossRef]

349. Afolayan, A.J.; Mbaebie, B.O. Ethnobotanical study of medicinal plants used as anti-obesity remedies in Nkonkobe Municipality of South Africa. Pharmacogn. J. 2010, 2, 368-373. [CrossRef]

350. Davids, D.; Gibson, D.; Johnson, Q. Ethnobotanical survey of medicinal plants used to manage High Blood Pressure and Type 2 Diabetes Mellitus in Bitterfontein, Western Cape Province, South Africa. J. Ethnopharmacol. 2016, 194, 755-766. [CrossRef]

351. Hulley, I.M.; Van Wyk, B.-E. Quantitative medicinal ethnobotany of Kannaland (western Little Karoo, South Africa): Nonhomogeneity amongst villages. S. Afr. J. Bot. 2019, 122, 225-265. [CrossRef]

352. Klos, M.; van de Venter, M.; Milne, P.J.; Traore, H.N.; Meyer, D.; Oosthuizen, V. In vitro anti-HIV activity of five selected South African medicinal plant extracts. J. Ethnopharmacol. 2009, 124, 182-188. [CrossRef] [PubMed]

353. Maphosa, V.; Masika, P.J.; Bizimenyera, E.S.; Eloff, J.N. In vitro anthelminthic activity of crude aqueous extracts of Aloe ferox, Leonotis leonurus and Elephantorrhiza elephantina against Haemonchus contortus. Trop. Anim. Health Prod. 2010, 42, 301-307. [CrossRef] 
354. Maphosa, V.; Masika, P.J. In vivo validation of Aloe ferox (Mill), Elephantorrhiza elephantina Bruch. Skeels. and Leonotis leonurus (L) R.Br. as potential anthelminthics and antiprotozoals against mixed infections of gastrointestinal nematodes in goats. Parasitol. Res. 2012, 110, 103-108. [CrossRef]

355. Odei-Addo, F.; Shegokar, R.; Müller, R.H.; Levendal, R.A.; Frost, C. Nanoformulation of Leonotis leonurus to improve its bioavailability as a potential antidiabetic drug. 3 Biotech 2017, 7. [CrossRef] [PubMed]

356. Mnonopi, N.; Levendal, R.A.; Davies-Coleman, M.T.; Frost, C.L. The cardioprotective effects of marrubiin, a diterpenoid found in Leonotis leonurus extracts. J. Ethnopharmacol. 2011, 138, 67-75. [CrossRef]

357. Mnonopi, N.; Levendal, R.A.; Mzilikazi, N.; Frost, C.L. Marrubiin, a constituent of Leonotis leonurus, alleviates diabetic symptoms. Phytomedicine 2012, 19, 488-493. [CrossRef]

358. Popoola, O.K.; Elbagory, A.M.; Ameer, F.; Hussein, A.A. Marrubiin. Molecules 2013, 18, 9049-9069. [CrossRef]

359. Roberts, M. Indigenous Healing Plants; Southern Book Publishers: Halfway House, South Africa, 1992 ; ISBN 1875093826.

360. Rood, B. Uit Die Veld-Apteek; Tafelberg: Cape Town, South Africa, 1994; ISBN 1869192745.

361. Batten, A.; Bokelman, H. Wildflowers of the Eastern Cape; Books of Africa: Cape Town, South Africa, 1996; ISBN B0006BSHIA.

362. De Oliveira, D.P.; De Almeida, L.; Marques, M.J.; De Carvalho, R.R.; Dias, A.L.T.; Da Silva, G.A.; De Pádua, R.M.; Braga, F.C.; Da Silva, M.A. Exploring the bioactivity potential of Leonotis nepetifolia: Phytochemical composition, antimicrobial and antileishmanial activities of extracts from different anatomical parts. Nat. Prod. Res. 2019, 1-6. [CrossRef]

363. Hulley, I.M. Medicinal Ethnobotany of the Little Karoo, South Africa. Ph.D. Thesis, University of Johannesburg, Johannesburg, South Africa, 2018.

364. Mhlongo, L.S.; Van Wyk, B.-E. Zulu medicinal ethnobotany: New records from the Amandawe area of KwaZulu-Natal, South Africa. S. Afr. J. Bot. 2019, 122, 266-290. [CrossRef]

365. Rodin, R.J. The Ethnobotany of the Kwanyama Ovambos. Monographs in Systematic Botany 9; Missouri Botanical Garden: St. Louis, MO, USA, 1985; ISBN 999516843X.

366. Borah, M.; Das, S. Antidiabetic, antihyperlipidaemic and antioxidant activities of leaves of Leucas linifolia Spreng In Streptozocin induced diabetic rats. Eur. J. Biomed. Pharm. Sci. 2015, 2, 538-551.

367. Chandrashekar, K.S.; Prasanna, K.S.; Joshi, A.B. Hepatoprotective activity of the Leucas lavandulaefolia on d(+)galactosamineinduced hepatic injury in rats. Fitoterapia 2007, 78, 440-442. [CrossRef]

368. Ramalingam, R.; Nath, A.R.; Madhavi, B.B.; Nagulu, M. In vitro free radical scavenging, cytotoxic and acetylcholinesterase inhibitory activities of Leucas martinicensis. Int. J. Chem. Anal. Sci. 2013, 4, 91-95. [CrossRef]

369. Palmer, E. A Gardener's Year; Tafelberg: Cape Town, South Africa, 1995; ISBN 0624034062.

370. Thibane, V.S.; Ndhlala, A.R.; Abdelgadir, H.A.; Finnie, J.F.; Van Staden, J. The cosmetic potential of plants from the Eastern Cape Province traditionally used for skincare and beauty. S. Afr. J. Bot. 2019, 122, 475-483. [CrossRef]

371. Lodhi, S.; Vadnere, G.P.; Sharma, V.K.; Usman, M.R. Marrubium vulgare L.: A review on phytochemical and pharmacological aspects. J. Intercult. Ethnopharmacol. 2017, 6, 429-452. [CrossRef]

372. Yabrir, B. Essential oil of Marrubium vulgare: Chemical composition and biological activities. A review. Nat. Prod. Sci. 2019, 25, 81-91. [CrossRef]

373. De Beer, J.J.J.; Van Wyk, B.-E. An ethnobotanical survey of the Agter-Hantam, Northern Cape Province, South Africa. S. Afr. J. Bot. 2011, 77, 741-754. [CrossRef]

374. Thring, T.S.A.; Weitz, F.M. Medicinal plant use in the Bredasdorp/Elim region of the Southern Overberg in the Western Cape Province of South Africa. J. Ethnopharmacol. 2006, 103, 261-275. [CrossRef]

375. McGaw, L.J.; Jäger, A.K.; Van Staden, J. Prostaglandin synthesis inhibitory activity in Zulu, Xhosa and Sotho medicinal plants. Phyther. Res. 1997, 11, 113-117. [CrossRef]

376. Al-Bayati, F.A. Isolation and identification of antimicrobial compound from Mentha longifolia L. leaves grown wild in Iraq. Ann. Clin. Microbiol. Antimicrob. 2009, 8. [CrossRef]

377. Akroum, S.; Bendjeddou, D.; Satta, D.; Lalaoui, K. Antibacterial activity and acute toxicity effect of flavonoids extracted from Mentha longifolia. Am. J. Sci. Res. 2009, 4, 93-96.

378. Smith, C.A. Common Names of South African Plants. Memoirs of the Botanical Survey of South Africa No. 35; Department of Agriculture and Technical Services, Pretoria: Pretoria, South Africa, 1966; ISBN 5016872-1963-64-2,500.

379. Van den Eynden, V.; Vernemmen, P.; Van Damme, P. The Ethnobotany of the Topnaar; Universiteit Gent: Gent, Belgium, 1992; ISBN B00J2HBINK.

380. Carović-Stanko, K.; Orlić, S.; Politeo, O.; Strikić, F.; Kolak, I.; Milos, M.; Satovic, Z. Composition and antibacterial activities of essential oils of seven Ocimum taxa. Food Chem. 2010, 119, 196-201. [CrossRef]

381. Cavalcanti, E.S.B.; de Morais, S.M.; Lima, M.A.A.; Santana, E.W.P. Larvicidal activity of essential oils from Brazilian plants against Aedes aegypti L. Mem. Inst. Oswaldo Cruz 2004, 99, 541-544. [CrossRef] [PubMed]

382. Fawole, O.A.; Finnie, J.F.; Van Staden, J. Antimicrobial activity and mutagenic effects of twelve traditional medicinal plants used to treat ailments related to the gastro-intestinal tract in South Africa. S. Afr. J. Bot. 2009, 75, 356-362. [CrossRef]

383. Mhlongo, L.S. The Medicinal Ethnobotany of the Amandawe Area in KwaCele, KwaZulu-Natal, South Africa. Master's Thesis, University of Johannesburg, Johannesburg, South Africa, 2019. 
384. Figueiredo, N.L.; de Aguiar, S.R.M.M.; Falé, P.L.; Ascensão, L.; Serralheiro, M.L.M.; Lino, A.R.L. The inhibitory effect of Plectranthus barbatus and Plectranthus ecklonii leaves on the viability, glucosyltransferase activity and biofilm formation of Streptococcus sobrinus and Streptococcus mutans. Food Chem. 2010, 119, 664-668. [CrossRef]

385. Boon, R. Pooley's Trees of Eastern South Africa. A Complete Guide, 2nd ed.; Natal Flora Publications Trust: Durban, South Africa, 2010; ISBN 9780620460194.

386. Njeru, S.N.; Obonyo, M.; Nyambai, S.; Ngari, S.; Mwakubambanya, R.; Mavura, H. Antimicrobial and cytotoxicity properties of the organic solvent fractions of Clerodendrum myricoides (Hochst.) R. Br. ex Vatke: Kenyan traditional medicinal plant. J. Intercult. Ethnopharmacol. 2016, 5, 226-232. [CrossRef] [PubMed]

387. Chege, B.M.; Waweru, M.P.; Frederick, B.; Nyaga, N.M. The freeze-dried extracts of Rotheca myricoides (Hochst.) Steane and Mabb possess hypoglycemic, hypolipidemic and hypoinsulinemic on type 2 diabetes rat model. J. Ethnopharmacol. 2019, $244,112077$. [CrossRef]

388. Hayelom, K.; Mekbeb, A.; Eyasu, M.; Wondwossen, E.; Kelbesa, U. Methanolic effect of Clerodendrum myricoides root extract on blood, liver and kidney tissues of mice. Afr. Health Sci. 2012, 12, 489-497. [CrossRef]

389. Van Wyk, B.-E. A review of Khoi-San and Cape Dutch medical ethnobotany. J. Ethnopharmacol. 2008, 119, 331-341. [CrossRef]

390. Motlhatlego, K.E. Evaluation of Plants Used in African Traditional Medicine for Asthma and Related Conditions. Ph.D. Thesis, University of KwaZulu-Natal, Pietermaritzburg, South Africa, 2014.

391. Cock, I.E.; Van Vuuren, S.F. The potential of selected South African plants with anti-Klebsiella activity for the treatment and prevention of Ankylosing spondylitis. Inflammopharmacology 2014, 23, 21-35. [CrossRef]

392. Jäger, A.K.; Van Staden, J. Salvia in southern Africa. In Sage: The genus Salvia; Kintzios, S.E., Ed.; Harwood Academic Publishers: Amsterdam, The Netherlands, 2000; ISBN 0203304551.

393. Nortje, J.M.; Van Wyk, B.-E. Useful plants of Namaqualand, South Africa: A checklist and analysis. S. Afr. J. Bot. 2019, 122, 120-135. [CrossRef]

394. Mössmer, N.; David, L. Traditional Herbal Remedies; Montagu Museum: Montagu, South Africa, 2019.

395. Smith, A. A Contribution to South African Materia Medica; Juta: Cape Town, South Africa, 1895; ISBN 978-0-86810-469-0.

396. Shearing, D. Karoo. South African wild flower guide 6; Botanical Scociety of South Africa: Kirstenbosch, Cape Town, South Africa, 1994; ISBN 9781874999041.

397. Elgorashi, E.E.; Taylor, J.L.S.; Maes, A.; Van Staden, J.; De Kimpe, N.; Verschaeve, L. Screening of medicinal plants used in South African traditional medicine for genotoxic effects. Toxicol. Lett. 2003, 143, 195-207. [CrossRef]

398. Coopoosamy, R.M.; Naidoo, K.K. Assessing the potential of Tetradenia riparia in treatment of common skin conditions in rural communities of South Africa. African J. Microbiol. Res. 2011, 5, 2942-2945. [CrossRef]

399. Akhalwaya, S.; Van Vuuren, S.; Patel, M. An in vitro investigation of indigenous South African medicinal plants used to treat oral infections. J. Ethnopharmacol. 2018, 210, 359-371. [CrossRef]

400. Endo, E.H.; Costa, G.M.; Nakamura, T.U.; Nakamura, C.V.; Dias Filho, B.P. Antidermatophytic activity of hydroalcoholic extracts from Rosmarinus officinalis and Tetradenia riparia. J. Mycol. Med. 2015, 25, 274-279. [CrossRef]

401. Endo, E.H.; Costa, G.M.; Makimori, R.Y.; Ueda-Nakamura, T.; Nakamura, C.V.; Dias Filho, B.P. Anti-biofilm activity of Rosmarinus officinalis, Punica granatum and Tetradenia riparia against methicillin-resistant Staphylococcus aureus (MRSA) and synergic interaction with penicillin. J. Herb. Med. 2018, 14, 48-54. [CrossRef]

402. Ghuman, S.; Ncube, B.; Finnie, J.F.; McGaw, L.J.; Coopoosamy, R.M.; Van Staden, J. Antimicrobial activity, phenolic content, and cytotoxicity of medicinal plant extracts used for treating dermatological diseases and wound healing in KwaZulu-Natal, South Africa. Front. Pharmacol. 2016, 7, 320. [CrossRef]

403. Campbell, W.E.; Gammon, D.W.; Smith, P.; Abrahams, M.; Purves, T.D. Composition and antimalarial activity in vitro of the essential oil of Tetradenia riparia. Planta Med. 1997, 63, 270-272. [CrossRef] [PubMed]

404. Jarić, S.; Mitrović, M.; Pavlović, P. Ethnobotanical features of Teucrium species. In Teucrium Species: Biology and Applications; Stanković, M., Ed.; Springer International Publishing: Cham, Switzerland, 2020; pp. 111-142, ISBN 978-3-030-52159-2.

405. McGaw, L.J.; Eloff, J.N. Ethnoveterinary use of southern African plants and scientific evaluation of their medicinal properties. J. Ethnopharmacol. 2008, 119, 559-574. [CrossRef] [PubMed]

406. Stefanović, O. Antibacterial and antifungal activity of secondary metabolites of Teucrium species. In Teucrium Species: Biology and Applications; Stanković, M., Ed.; Springer International Publishing: Cham, Switzerland, 2020; pp. 319-354, ISBN 978-3-030-52159-2.

407. Coats Palgrave, K. Trees of Southern Africa; Struik Publishers: Cape Town, South Africa, 1977; ISBN 1868251713.

408. McGaw, L.J.; Jäger, A.K.; Staden, J. Van Antibacterial, anthelmintic and anti-amoebic activity in South African medicinal plants. J. thnopharmacol. 2000, 72, 247-263. [CrossRef]

409. Adamu, M.; Naidoo, V.; Eloff, J.N. Some southern African plant species used to treat helminth infections in ethnoveterinary medicine have excellent antifungal activities. BMC Complement. Altern. Med. 2012, 12. [CrossRef] [PubMed]

410. Waller, S.B.; Cleff, M.B.; Serra, E.F.; Silva, A.L.; dos Gomes, A.R.; de Mello, J.R.B.; de Faria, R.O.; Meireles, M.C.A. Plants from Lamiaceae family as source of antifungal molecules in humane and veterinary medicine. Microb. Pathog. 2017, 104, $232-237$. [CrossRef] [PubMed]

411. Bapela, M.J.; Meyer, J.J.M.; Kaiser, M. In vitro antiplasmodial screening of ethnopharmacologically selected South African plant species used for the treatment of malaria. J. Ethnopharmacol. 2014, 156, 370-373. [CrossRef] 
412. Bapela, M.J.; Kaiser, M.; Meyer, J.J.M. Antileishmanial activity of selected South African plant species. S. Afr. J. Bot. 2017, 108, 342-345. [CrossRef]

413. Kunkel, G. Plants Africa gave to the world. Bothalia 1983, 14, 465-469. [CrossRef]

414. Asfaw, Z.; Tadesse, M. Prospects for sustainable use and development of wild food plants in Ethiopia. Econ. Bot. 2001, 55, 47-62. [CrossRef]

415. Van Wyk, B.-E.; Gericke, N. People's Plants: A Guide to Useful Plants of Southern Africa, 2nd ed.; Briza Publications: Pretoria, South Africa, 2017; ISBN 9781920217716.

416. Crouch, N.R.; Styles, D.G.A. Rediscovery in South Africa of the Neglected African Vegetable Plectranthus Esculentus. Bothalia 2010, 40, 65-67. [CrossRef]

417. Rice, L.J.; Brits, G.J.; Potgieter, C.J.; Van Staden, J. Plectranthus: A plant for the future? S. Afr. J. Bot. 2011, 77, 947-959. [CrossRef]

418. Welcome, A.K.; Van Wyk, B.-E. An inventory and analysis of the food plants of southern Africa. S. Afr. J. Bot. 2019, 122, 136-179. [CrossRef]

419. Magwede, K.; Van Wyk, B.-E.; Van Wyk, A.E. An inventory of Vhavenda useful plants. S. Afr. J. Bot. 2019, 122, 57-89. [CrossRef]

420. Welcome, A.K. Food Plants of Southern Africa. Ph.D. Thesis, University of Johannesburg, Johannesburg, South Africa, 2019.

421. Nortje, J.M. Ethnobotany of Namaqualand, South Africa. Ph.D. Thesis, University of Johannesburg, Johannesburg, South Africa, 2019.

422. Malan, J.S.; Owen-Smith, G.L. The ethnobotany of Kaoland. In Proceedings of the Cimbeasia. 3.2.5.; Indiana University: Bloomington, IN, USA, 1974; pp. 131-178.

423. Archer, F.M. Planning with people—Ethnobotany and African uses of plants in Namaqualand (South Africa). In Proceedings of the 12th Plenary Meeting of AEFAT, Hamburg, Germany, 4-10 September 1988; pp. 959-972.

424. Archer, F.M. Ethnobotany of Namaqualand: The Richtersveld. Master's Thesis, University of Cape Town, Cape Town, South Africa, 1994.

425. Van Breda, P.A.B.; Barnard, S.A. 100 Veld Plants of the Winter Rainfall Region; Department of Agricultural Development: Elsenburg, South Africa, 1991; ISBN 0-621-13470-8.

426. Viljoen, A.M.; Van Vuuren, S.; Ernst, E.; Klepser, M.; Demirci, B.; Başer, H.; Van Wyk, B.-E. Osmitopsis asteriscoides (Asteraceae)— The antimicrobial activity and essential oil composition of a Cape-Dutch remedy. J. Ethnopharmacol. 2003, 88, 137-143. [CrossRef] 